\title{
Detector development for Positron Emission based real-time Tumor Tracking
}

by

Mojgan Soleimani, M.Sc.

\author{
A Thesis Submitted to \\ The Faculty of Graduate Studies and Research \\ In Partial Fulfillment of \\ The Requirement for the Degree of \\ Master of Science
}

Ottawa-Carleton Institution for Physics

Department of Physics, Carleton University

Ottawa, Ontario, Canada

Submitted July, 2009

Ccopyright

2009, Mojgan Soleimani 


\begin{abstract}
Tumor motion limits the accuracy of radiation therapy. Positron emission tracking (PeTrack) is a technique that can track tumors through detecting annihilation gammas from implanted positron emission markers. This thesis focuses on detector development for PeTrack. Due to the intense scattered x rays from a Linac, scintillator (BGO) afterglow and detector gating, i.e. turning off the detector during the intense $\mathrm{x}$-ray pulse, need to be addressed. The evaluation of BGO showed very low afterglow. A gating circuit was designed, optimized and tested. Energy resolution of the detector is better than 25\% (FWHM) with optimal gating parameters. A data acquisition system for PeTrack was set up and calibrated successfully. The first PeTrack prototype was developed and evaluated. The prototype was able to localize two positron emitting markers with an average precision of $0.16-0.21 \mathrm{~mm}$, and an average accuracy of $0.6 \mathrm{~mm}$ on distance between the two markers.
\end{abstract}




\section{Acknowledgements}

This work is dedicated to my parents, who encouraged me to explore my academic interests and curiosities and have led me to where I am today. And to my dear husband who has always stood by my side and without his support this work could not have been completed. And to my sister, her little son, and my brother, whose support and encouragement made my intellectual journey a better experience.

I would like to express my gratitude to my supervisor, Dr. Tong Xu, for his continuous support and commitment to his students, and also for his wide knowledge, valuable advice and excellent guidance throughout this project.

I would also like to thank Malcolm McEwen from the National Research Council of Canada who facilitated the use of the clinical linear accelerator at NRC.

I am also deeply grateful to my friend, Benjamin Spencer, for his extreme help and support in computer programming, designing figures and plotting graphs for this project.

I warmly thank my lab partner, Marc Chamberland, for the information he provided me, with regard to PeTrack algorithm.

I would also like to thank Dr. Shengli Liu, Yves Baribeau, and Cliff Hargrove, for consultation on gating circuit design and electronics.

I also wish to thank, Philippe Gravelle, for his help in machining the housing of detectors.

I would also like to thank, Bill Jack, for his assistance in software installation.

I would also like to acknowledge NSERC Discovery Grant for funding this project. 


\section{Table of Contents}

Abstract.................................................................................................................. ii

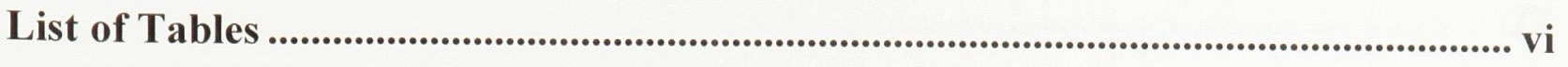

List of Figures......................................................................................................... vii

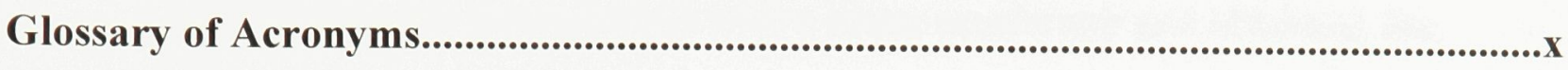

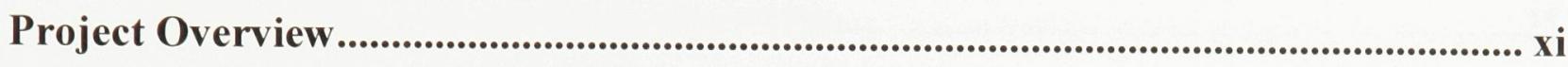

Chapter 1 - Introduction (Background) .....................................................................1

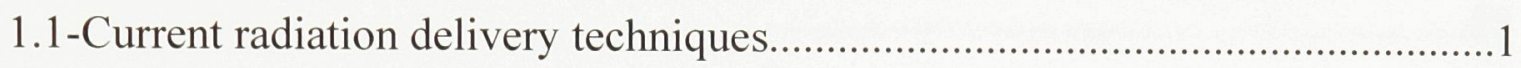

1.2-Limitation of radiation delivery ............................................................

1.3 -Current tumor tracking techniques during radiation therapy ........................4

1.4-PeTrack: A new tumor tracking technique ................................................5

Chapter 2- Design of the PeTrack system...........................................................................7

2.1-Principles of PeTrack....................................................................... 7

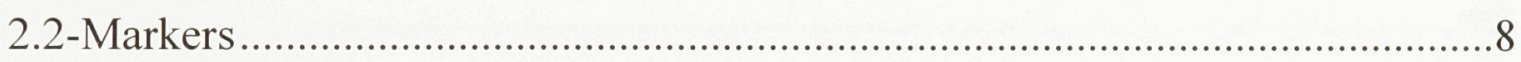

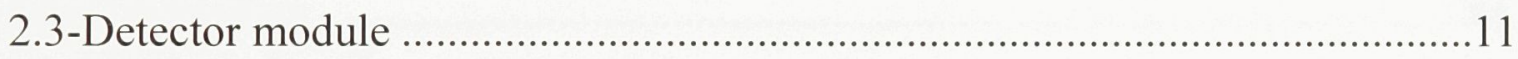

2.4-Readout system ......................................................................... 15

2.5-Single marker and multi-marker tracking algorithm ...............................16

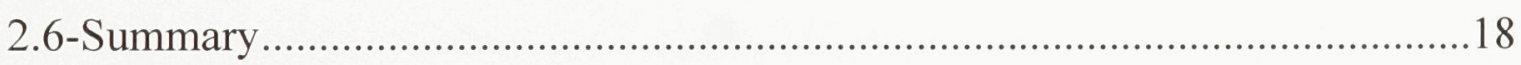

Chapter 3 - BGO Crystal.........................................................................................19

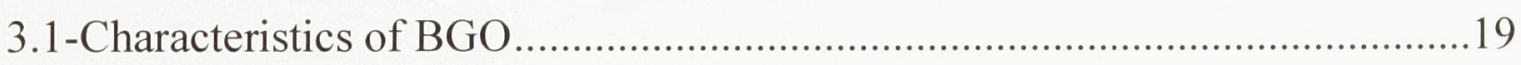

3.2-Radiation Hardness .................................................................... 19

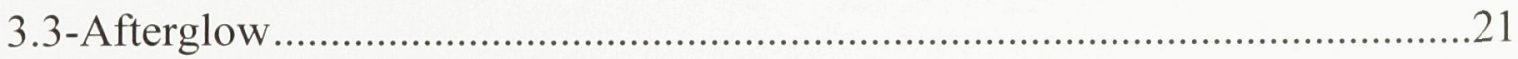

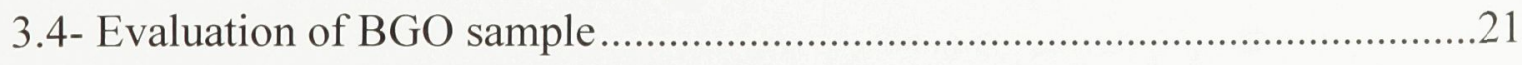


Chapter 4 - Gating of PeTrack detector........................................................................27

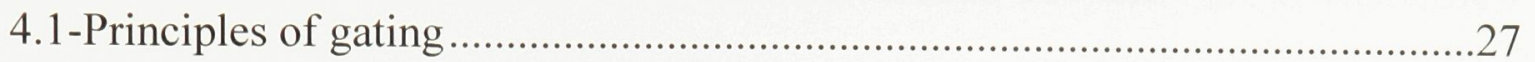

4.2-Evaluation of the gating circuit with UV excitation.......................................31

4.3-Evaluating the gating circuit with a linear accelerator and obtaining the

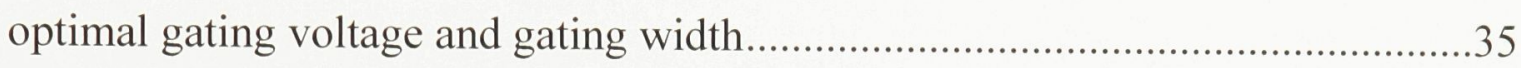

4.4-Evaluation of gating with optimized parameters …………..............................

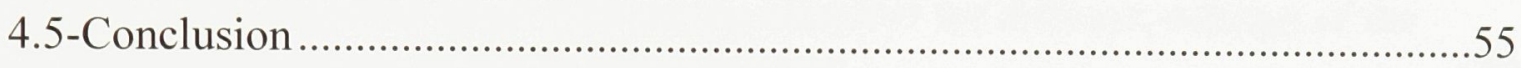

Chapter 5 - The first PeTrack prototype .............................................................56

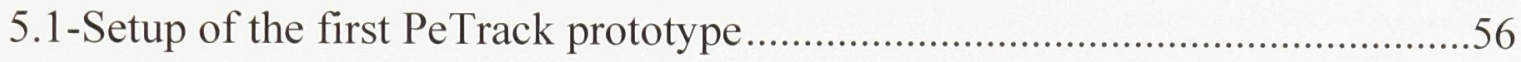

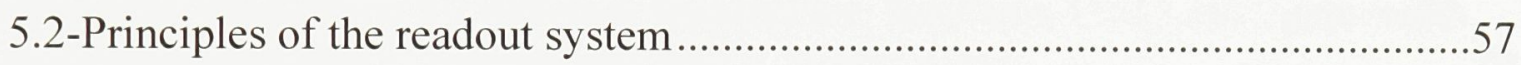

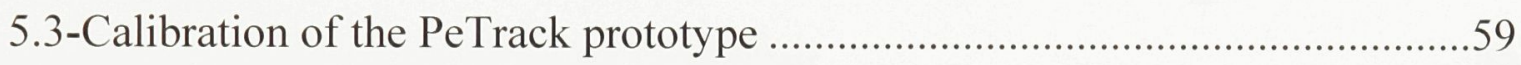

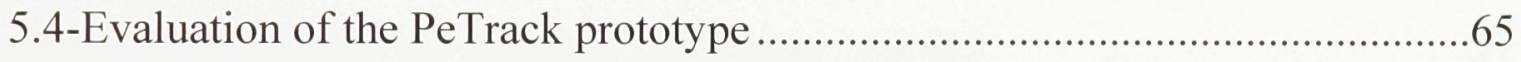

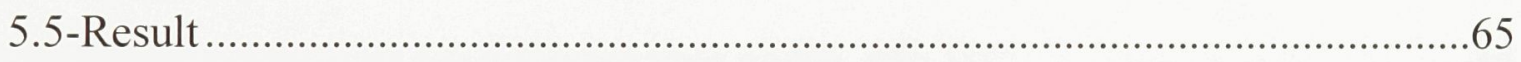

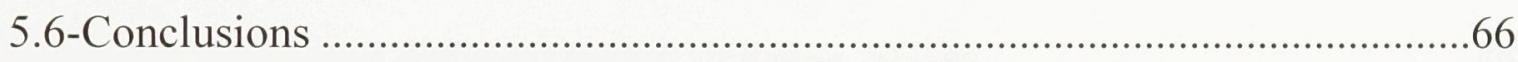

Chapter 6 - Conclusion and future work .................................................................67

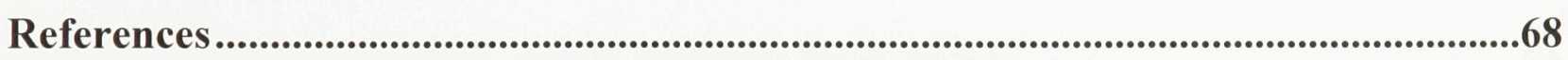




\section{List of Tables}

Table 2.1 Average positron energy (keV) for isotopes used in PeTrack 9

Table 2.2 CSDA range of positron in different materials 9

Table 2.3 Life time dose at different distances from the marker with different isotopes (activity=3.7 MBq) [1]. 10

Table 3.1 Comparison of major inorganic scintillators

Table 3.2 Dose measurements in air with an ion chamber for different settings of the

Linac and different locations of the PeTrack detector. 20

Table 4.1 Energy resolutions for different frequencies: field size $15 \times 15 \mathrm{~cm}^{2}$, gate width $150 \mu \mathrm{s}$, gating voltage $200 \mathrm{~V}$, pmt voltage $830 \mathrm{~V}, 10 \mathrm{MV}$ beam

Table 4.2 Energy resolutions for different frequencies: field size $15 \times 15 \mathrm{~cm}^{2}$, gate width $150 \mu \mathrm{s}$, gating voltage $200 \mathrm{~V}$, pmt voltage $830 \mathrm{~V}, 6 \mathrm{MV}$ beam

Table 4.3 Energy resolution for different field sizes .40

Table 4.4 Energy resolution for different gate width, $200 \mathrm{~Hz}$

Table 4.5 Energy resolutions for different gate width, $400 \mathrm{~Hz}$ 44

Table 4.6 Energy resolution different gating voltages, $50 \mathrm{~Hz}$. .45

Table 4.7 Energy resolution different gating voltages, $100 \mathrm{~Hz}$. .46

Table 4.8 Energy resolution, $6 \mathrm{MV}$, different frequencies .53

Table 4.9 Energy resolution, $10 \mathrm{MV}$, different frequencies .54

Table 5.1 energy resolutions for different crystal element in a 9x9 array of $4 \times 4 \mathrm{~mm}^{2}$ crystal elements. 


\section{List of Figures}

Figure 1.4.1 Positron emission and annihilation. 6

Figure 2.1.1 Drawing of four PeTrack detector modules (A1, A2, B1, B2) mounted on the linear accelerator gantry with $50 \mathrm{~cm}$ from isocentre .7

Figure 2.2.1 The positron emission marker for PeTrack system .......................................8

Figure 2.3.1 Schematics of PeTrack Detector modules..... 11

Figure 2.3.2 A $5.2 \times 5.2 \mathrm{~cm}^{2}$ BGO crystal pixelated to $13 \times 13$ array of $4 \times 4 \mathrm{~mm}^{2}$ elements and an H8500 Hamamatsu photomultiplier 12

Figure 2.3.3 Electrode structure and electron trajectory of a Position Sensitive PMT......13 Figure 2.3.4 a: Anode pad array of PMT with the position coordinates x, y, b: Active resistive network and four output signals 14

Figure 3.4.1 A $2 \times 2 \times 2 \mathrm{~cm}^{3}$ undoped BGO crystal 21

Figure 3.4.2 Detector unit (a), electronic schematics (b), used for primary evaluations. Crystal is coupled to the PMT with silicon grease, outer cap moves and covers the housing .22

Figure 3.4.3 Showing the intensity of the UV light shining on a $2 \times 2 \times 2 \mathrm{~cm}^{3} \mathrm{BGO}$ 23

Figure 3.5.1 UV signal, top, and PMT output at 500V, bottom 25

Figure 3.5.2 Energy resolution of BGO for $511 \mathrm{keV}$ gamma rays, high voltage 830 volts .25

Figure 3.5.3 PMT output due to scattered $\mathrm{x}$ rays from a Linac at different field sizes .....26 Figure 4.1.1 Schematics of gating circuit. $\mathrm{K}$ is the photocathode. DY1...DY10 are PMT dynodes $1 \ldots 10 . \mathrm{P}$ is the anode. HV1 is the high voltage power supply for the PMT. HV2 is the gating voltage. GATE is the input for gating pulse 28 
Figure 4.1.2 a: Gate signal, $10 \mu \mathrm{sec}$, b: Dynode 2, 3, 4, and 5 voltages before, during and after gate signal 30

Figure 4.2.1 UV LED signal (top) and gating signal (bottom) ...................................

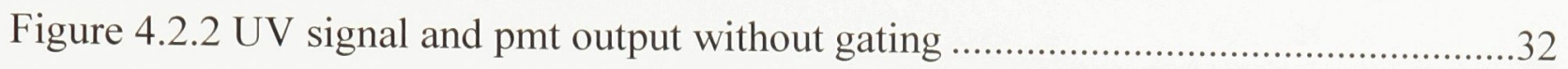

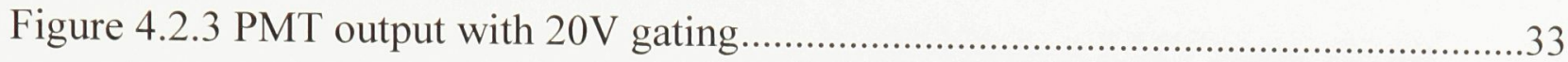

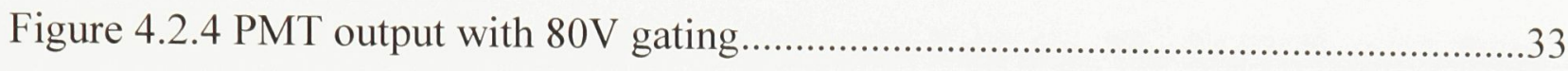

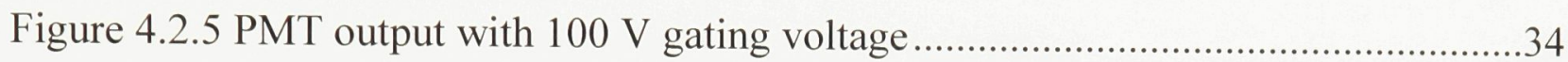

Figure 4.3.1 Geometry (a), and electronics schematics (b), of the detector unit setup with

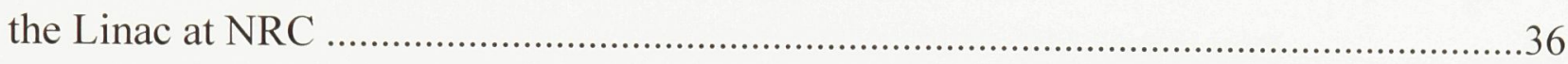

Figure 4.3.2 Energy spectrum of ${ }^{22} \mathrm{Na}$, in the radiation environment of a Linac, with

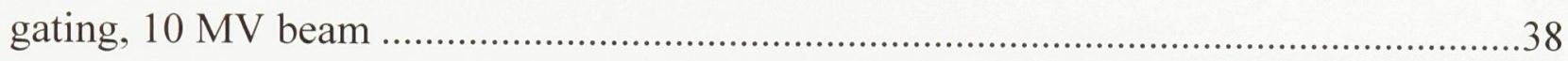

Figure 4.3.3 Energy spectrum of ${ }^{22} \mathrm{Na}$, in the radiation environment of a Linac, with gating, $6 \mathrm{MV}$ beam 39

Figure 4.3.4 Energy spectrum of ${ }^{22} \mathrm{Na}$, with gating, $6 \mathrm{MV}$ beam, different field sizes 40

Figure 4.3.5 Energy spectrum of ${ }^{22} \mathrm{Na}$, with gating, $6 \mathrm{MV}$ beam,

different gate width

Figure 4.3.6 Energy spectrum of ${ }^{22} \mathrm{Na}, 150 \mu$ s gating, $6 \mathrm{MV}$ beam, $400 \mathrm{~Hz}$ x-ray signal. 42

Figure 4.3.7 Energy spectrum of ${ }^{22} \mathrm{Na}, 50 \mu$ s gating, $6 \mathrm{MV}$ beam, $400 \mathrm{~Hz}$ x-ray signal .43

Figure 4.3.8 Energy spectrum of ${ }^{22} \mathrm{Na}, 6 \mathrm{MV}$ beam, $400 \mathrm{~Hz}$ x-ray signal, different gate width 
Figure 4.3.9 Energy spectrum of ${ }^{22} \mathrm{Na}$ for different gating voltages, $50 \mathrm{~Hz}$. 45

Figure 4.3.10 Energy spectrum of ${ }^{22} \mathrm{Na}$ for different gating voltages, $100 \mathrm{~Hz}$.............46

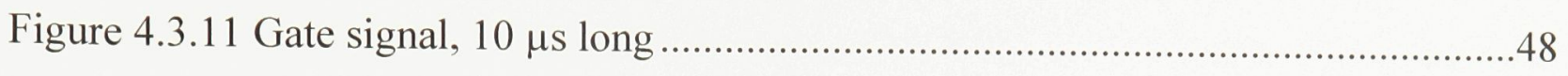

Figure 4.3.12 PMT output signal for different gating voltages ..................................48

Figure 4.3.13 (a) PMT output signal for different field sizes at $6 \mathrm{MV}$ beam. (b) the amplitude of the PMT signal was plotted against the field size. The line indicates a linear regressing fit. The gating parameter used are $100 \mathrm{~V}$ and $10 \mu \mathrm{s}$

Figure 4.3.14 PMT output signal for different field sizes at $10 \mathrm{MV}$ beam......................50

Figure 4.3.15 PMT output for 6 and $10 \mathrm{MV}$ beams at $45^{\circ}$, forward scatter ...................51

Figure 4.3.16 PMT output for 6 and $10 \mathrm{MV}$ beam at $45^{\circ}$, backscatter...........................52

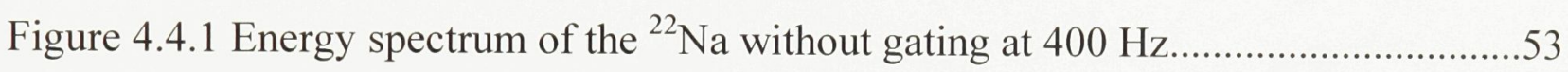

Figure 4.4.2 Energy spectrum of ${ }^{22} \mathrm{Na}$, at $6 \mathrm{MV}$ for different frequencies ......................54

Figure 4.4.3 Energy spectrum of ${ }^{22} \mathrm{Na}$, at $10 \mathrm{MV}$ for different frequencies ....................55

Figure 5.1.1 Schematics of the first PeTrack prototype.............................................56

Figure 5.2.1 (a) schematics of the readout system for PeTrack

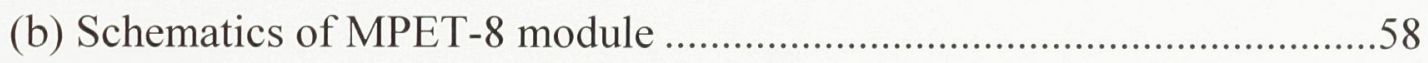

Figure 5.3.1 2D position histogram of PMT \# 0 ...................................................60

Figure 5.3.2 One dimensional profile of one row of crystal elements..........................61

Figure 5.3.3 Energy spectrum of $4 \times 4 \mathrm{~mm}^{2}$ crystal elements, (a) crystal ID number $(1,1)$,

(b) crystal ID number $(5,5)$, and (c) crystal ID number $(9,5)$ 63 


\section{Glossary of Acronyms}

$\mathrm{BGO}$ - bismuth germinate

BGO:Eu - europium doped BGO

CSDA - continuous-slowing-down approximation

CT - computed tomography

CTV - clinical tumor volume

3DCRT - three dimensional conformal radiation therapy

FWHM- full width half maximum

GTV - gross tumor volume

IMRT - intensity modulated radiation therapy

IMAT - intensity modulated arc therapy

MLC - multileaf collimator

MRI - magnetic resonance imaging

PEPT - positron emission particle tracking

PET - positron emission tomography

PM - Photomultiplier

PMT - Photomultiplier tube

PTV - planned tumor volume

SPECT - single photon emission computed tomography

US - ultrasound 


\section{Project overview and summary of contributions}

Radiation therapy is one of the main modalities for the localized treatment of tumors. Although there have been advancements in radiation delivery techniques, tumor motion can limit the accuracy of the radiation therapy. Patient movement, respiration, and transient physiological events, change the position of the treatment site during externalbeam radiotherapy. Studies have shown that tumor located in the chest or abdominal area can move up to $30 \mathrm{~mm}$ due to respiration or cardiac beating [1,2, and 3].

To achieve the goals of radiation therapy it is important to consider tumor's motion in radiation treatment planning. Present practice accommodates target movement by expanding the treatment beam to ensure that the treatment target always remains within the irradiated field. However, this increases the radiation exposure to healthy tissue, limiting the maximum dose that can be delivered to the tumor. Lower doses results in inferior treatment outcomes. In an ideal radiotherapy procedure the treatment system would continuously adapt the radiation beam delivery to compensate target movement, thus elimination motion margins and enabling higher doses.

Treatment during free breathing presents the most challenging problem in motionadaptive radiotherapy. There are presently two basic strategies to synchronize dose delivery to respiration: (1) turn the beam on only when the tumor is estimated to be at a specific position in the respiratory cycle (temporal gating); or (2) shift the beam in space to maintain alignment with the tumor throughout the respiratory cycle (spatial tracking). External or internal markers or other devices are used to monitor the respiratory motion. There are many factors that contribute to bad correlations between external markers and internal motion, such as unstable breathing, phase shift and cardiac beating, Therefore 
internal markers are preferable. Internal markers are implanted in or close to tumor. The techniques are invasive but provide exact location of the tumor. Two kind of internal markers are: Radio-opaque markers that can be tracked by two x-ray imaging systems and Electromagnet (EM) markers that can be tracked by an array of coils (antenna). Both of these tracking techniques have their own limitations. X-ray fluoroscopy introduces extra dose to the patient and electromagnetic tracking uses large size markers which can be dangerous to be implanted into lung tumors. With the Positron emission based tumor tracking technique (PeTrack), that was first introduced in 2006 by Dr. Tong Xu, et. al.[1], small radio opaque markers labeled with a short half life positron emission source that are implanted inside or around tumor will be tracked. Tracking is done with two pairs of position sensitive gamma detector modules. The objective of this work was to develop and evaluate the detector for PeTrack and also to set up and calibrate a prototype PeTrack system.

Bismuth Germinate crystal was chosen as scintillator for the detector. In the first part of this project I evaluated the characteristics, mainly afterglow, of our BGO crystal sample. To avoid overloading the PeTrack detector with scattered $\mathrm{x}$ rays from the linear accelerator, the PeTrack detector should be able to work in a gated mode, i.e. the detector should be turned off during the $\mathrm{x}$-ray pulse and be turned on between the $\mathrm{x}$-ray pulses to acquire positron emission signal. In this project, after the gating circuit for the detector was designed, I built and evaluated the gating circuit. I also tested the overall performance of the detector with the gating circuit. In the last part of the project, I constructed a prototype of a PeTrack system and evaluated its capability to localize the position of positron emission sources. 


\section{Chapter 1- Introduction}

In this chapter, overviews of different radiation delivery and tumor motion tracking techniques as well as their limitations are provided. These overviews will later lead to a brief introduction to the PeTrack technique.

\section{1- Current radiation delivery techniques}

Radiation therapy is one of the main modalities for the localized treatment of tumor. The goal of radiation therapy is to maximize the probability of tumor eradication and minimize the probability of injury to surrounding normal tissue. This can be achieved by delivering sufficient dose to tumor cells and reducing normal tissue dose to acceptable level. Recently, there have been significant improvements in radiation therapy delivery techniques.

\section{D conformal therapy (3DCRT)}

The three-dimensional conformal radiotherapy (3DCRT) process is based on a three dimensional computed tomography. Multiple beams from different directions are focused on the tumor. The shapes of the radiation fields are tailored to follow the outline of the tumor. This will result is a "conformed" radiation to tumor cells while avoiding normal adjacent tissue. Because higher doses of radiation can be delivered to cancer cells while significantly reducing the amount of radiation received by surrounding healthy tissues, the technique can increase the rate of tumor control while decreasing side effects.

\section{Multi-leaf collimator based intensity modulated radiation therapy (IMRT)}

Computer-controlled $\mathrm{x}$-ray accelerators are used in intensity-modulated radiation therapy(IMRT) [4]. IMRT delivers precise radiation doses that are conformed more precisely to the three-dimensional shape of the tumor by controlling the intensity of the 
radiation beam. With IMRT the radiation doses can be focused to regions within the tumor and therefore the dose to surrounding normal tissue is minimized. The treatment is planned by using 3-D CT images of the patient. A computerized dose calculation will determine the dose intensity pattern that will best conform to the tumor shape. A custom tailored radiation dose that maximizes tumor dose while protecting normal tissues surrounding the tumor will be produced by the combination of several intensitymodulated fields coming from different beam directions. Compared with 3DCRT, higher doses can safely be delivered to tumors while dose to normal adjacent tissue is reduced to minimum, with the IMRT approach.

\section{Tomotherapy}

Tomotherapy is delivery of intensity-modulated, rotational radiation therapy using a fan-beam delivery [5]. The NOMOS (Sewickley, PA) Peacock system is an example of sequential (or serial) tomotherapy that uses a fast-moving, actuator-driven multileaf collimator attached to a conventional $\mathrm{C}$-arm gantry to modulate the beam intensity. In helical tomotherapy, the patient is continuously translated through a ring gantry as the fan beam rotates. The beam delivery geometry is similar to that of helical computed tomography and requires the use of slip rings to transmit power and data.

\section{Intensity modulated arc therapy (IMAT)}

This technique combines spatial and temporal intensity modulation with the movement of the gantry [6]. Instead of using a slit (or fan) beam to treat a single slice of the patient at a time, as in tomotherapy or other slice-based treatments MLC-shaped fields, which change shape during gantry rotation, to deliver the dose to the treatment target are used. 


\section{Robot-based radiosurgery}

The CyberKnife Stereotactic Radiosurgery System [7] uses computer-assisted, non-surgical technology to transmit small beams of radiation from several different angles into a tumor thereby minimizing the exposure of surrounding healthy tissue. Based on CT scan data of a tumor, and the skeletal structure of the body as a reference frame, a high speed computer-controlled robotic arm is used to position the beam to follow patient and tumor positions during treatment.

\section{2- Limitation of radiation delivery}

The recent development of delivery techniques has significantly improved degree of dose conformity to the planning target volume. With the help of advanced imaging modalities (CT, MRI, SPECT, PET, and US) tumor visualization and eradication have improved a lot within the last few years [8]. For tumors located in the chest or abdominal areas though, the limiting factor on the delivery accuracy of radiation therapy is tumor's motion due to respiration or cardiac beating [9]. To cover tumor's motion during respiration larger target volume margin is used. This results in significant dose to the normal tissue surrounding the tumor. Applying precise respiration gating is one of the solutions and requires the exact spatial position of the tumor to be determined in real-time during treatment. Skin markers used in this technique can provide indirect indication of tumor position which can introduce significant tracking errors [10,11]. Even with implanted x-ray fiducial markers that are correlated with the external respiratory surrogates prior to each treatment fraction, due to the intra-fractional variation of the patient's breathing pattern the residual motion can still be large [11-13]. Real time tumor 
tracking can provide accurate respiration gating or even tumor-tracked radiation therapy.

\section{3 - Current tumour tracking techniques during radiation therapy}

To succeed, a tracking system must be able to do four things: (1) determine the tumor position; (2) anticipate the tumor motion to allow for time delays in realignment of the beam; (3) transmit the target coordinates to the re-alignment system through a control loop; (4) reposition the beam [14]. All of this must be done automatically and in real time, where real time refers to timescales that are short compared to the period over which the tumor moves appreciably. Tracking tumour position in real-time is the first step. Current tracking techniques include x-ray imaging and electromagnetic tracking.

\section{$X$-ray imaging}

If planar x-ray imaging is used, there is a challenge to see and automatically isolate low-contrast tumor tissue in the images [15]. This problem has been most often solved by implanting point-like radio-opaque markers in the tumor, which show up clearly in x-ray and can be quickly and automatically localized. If a fluoroscopic imaging system is used, a real-time stream of tumor position at $10-30 \mathrm{~Hz}$ can be observed. However, fluoroscopy during lengthy radiotherapy fractions delivers a significant concomitant radiation dose to the patient: 30-100 cGy skin dose per hour of treatment time over an area of $100-200 \mathrm{~cm}^{2}[17]$.

\section{Electromagnetic Tracking}

In this technique electromagnetic transponders are implanted at the treatment site [16]. The wireless transponders are activated by an external transmitter and then allowed to radiate EM signals at their characteristic frequency. The radiated signals are detected 
by an array of receiver antennae positioned above the patient, from which the system triangulates to the position of the transponder [14]. Implanting radiographic fiducials or transponders, typically $1.8 \mathrm{~mm} \times 8 \mathrm{~mm}$ cylinders, is an invasive procedure that has associated risk, especially in the lung.

\section{4- PeTrack: A new tumor tracking technique}

After discussing different radiation delivery techniques and their limitations, the positron emission tracking (PeTrack) technique is introduced in this section as a solution for the limitations mentioned above.

PeTrack uses very small $(0.5-0.8 \mathrm{~mm}$ diameter $)$ markers labeled with a very low activity (0.03-0.2 $\mathrm{mCi}$ ) positron emission source. By detecting pairs of annihilation gammas from the positron source, with two pairs of position sensitive gamma detectors that are installed onto the linear accelerator gantry, the position of the markers can be determined. Figure 1.4.1 shows the positron annihilation and two back-to-back gamma rays produced. The PeTrack technique can provide real-time tumor tracking with low dose to normal tissue and will increase the feasibility of precise respiration gated IMRT, tumor tracked radiation therapy $[18,19]$, and robotic motion compensated radiosurgery [4]. The implantation of positron emission markers, whose diameter can be as small as $0.5 \mathrm{~mm}$ diameter, is easier than implantation of existing markers. 


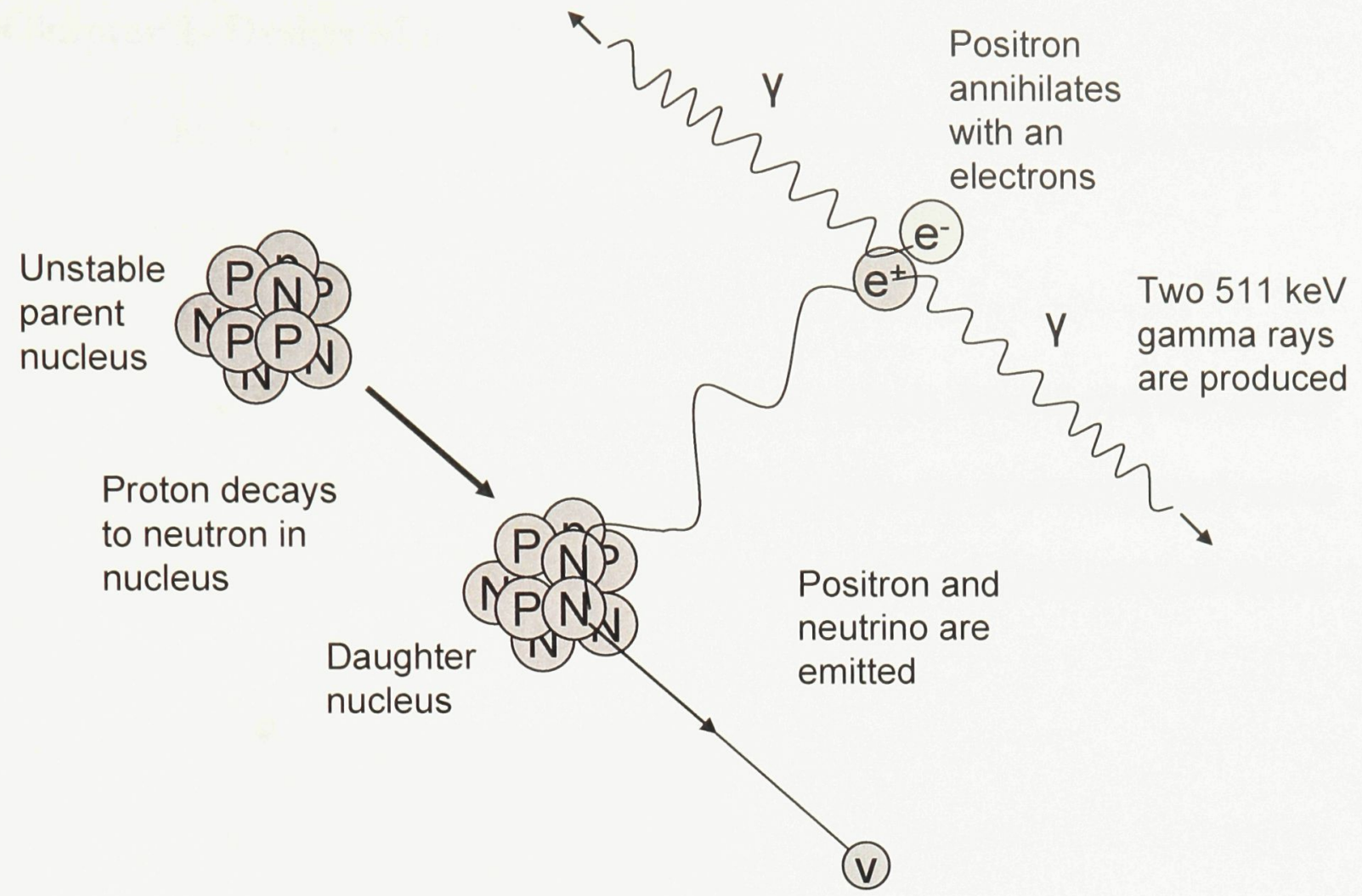

Figure 1.4.1 Positron emission and annihilation

In the following chapters, the design and evaluation of the PeTrack detectors will be discussed. The construction and evaluation of a PeTrack prototype will be also presented. 


\section{Chapter 2- Design of the PeTrack system}

In this chapter the proposed design of the PeTrack system including markers, detector modules, and readout systems will be discussed.

\subsection{Principle of PeTrack}

The PeTrack system uses positron emission markers, labeled with low activity positron emission isotopes, and position-sensitive gamma ray detectors to track tumor position in real time (figure 2.1.1). Two pairs of detector modules installed $50-70 \mathrm{~cm}$

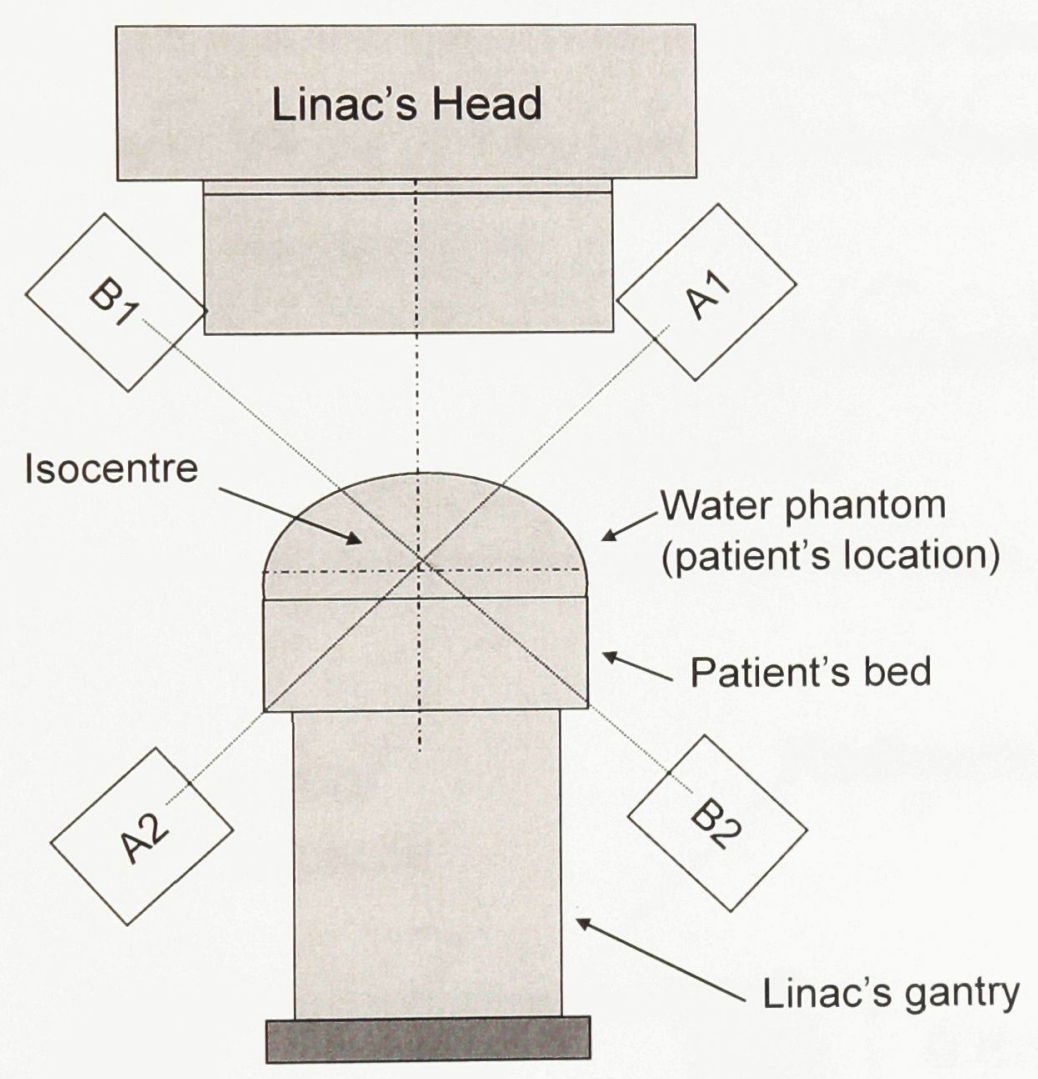

Figure 2.1.1 Drawing of four PeTrack detector modules (A1, A2, B1, B2) mounted on the linear accelerator gantry with $50 \mathrm{~cm}$ from isocentre [1].

from the isocentre of the linear accelerator, will detect back-to-back annihilation gammas from the positron source. By acquiring these events from each marker, coincidence lines between each pair of detectors are obtained. These coincidence lines are fed to a multi- 
marker localization algorithm which localizes the position of the markers in real time with sub-millimeter accuracy.

The PeTrack system includes positron emission markers, two pairs of orthogonal oriented detector modules, multi-marker localization algorithm, front end electronics, and data acquisition system.

\section{2- Markers}

PeTrack markers are small titanium or gold capsules, 0.5-0.8 mm diameter, 2-4 $\mathrm{mm}$ long containing positron isotopes with very low activity $(0.03-0.2 \mathrm{mCi})$ and half life comparable to the total time span of radiation therapy. The most probable isotopes are ${ }^{24} \mathrm{I}\left(\mathrm{t}_{1 / 2}=4.2\right.$ days $),{ }^{74} \mathrm{As}\left(\mathrm{t}_{1 / 2}=17.8\right.$ days $)$, and ${ }^{84} \mathrm{Rb}\left(\mathrm{t}_{1 / 2}=32\right.$ days $)$, which have already been used for PET imaging [20-23].

These small radio-opaque markers labeled with a positron emission source, mentioned above, will be implanted inside or around the tumor.

Figure 2.2.1 shows a positron emission marker for the PeTrack system.

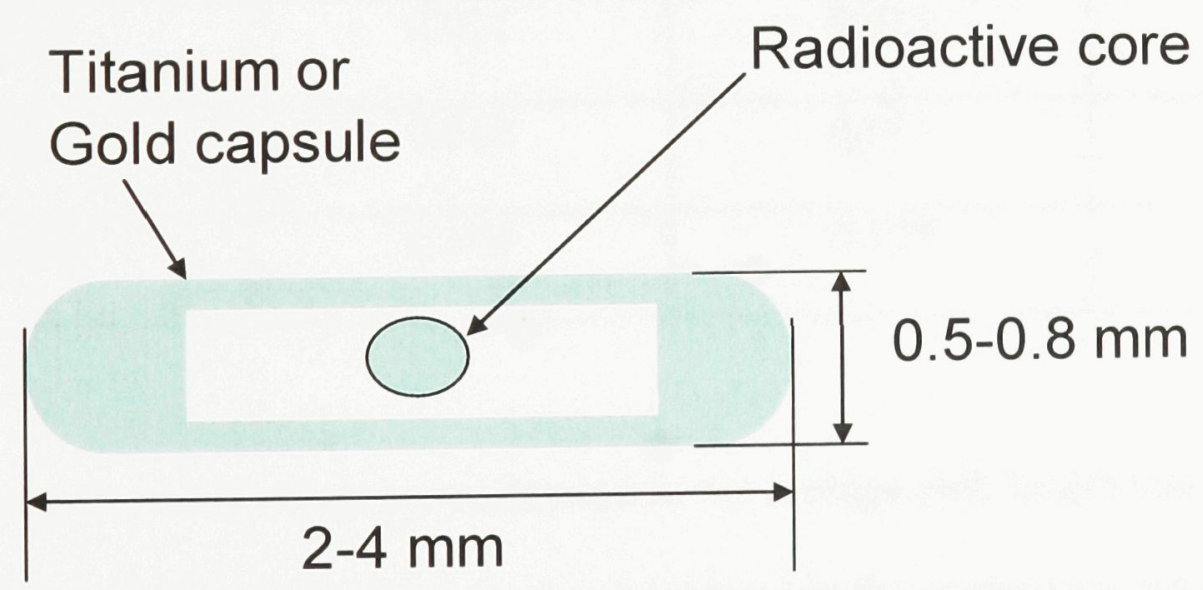

Figure 2.2.1 The positron emission marker for the PeTrack system 
Table 2.1 shows the average energy of the positron emitted from the mentioned isotopes, and Table 2.2 shows continuous slowing down approximation range, CSDA, in different materials.

Table 2.1 Average positron energy (keV) for isotopes to be used in PeTrack

\begin{tabular}{|c|c|c|c|}
\hline & ${ }^{74}$ As & ${ }^{84} R b$ & ${ }^{124} I$ \\
\hline Average positron & 336 & 408 & 604 \\
energy (keV) & & & \\
\hline
\end{tabular}

Average positron energy is a weighted average energy of the positrons emitted from the isotope.

Table 2.2 CSDA range of positrons in different materials

\begin{tabular}{|c|c|c|c|}
\hline \multicolumn{3}{|c|}{ CSDA range at average energy $(\mathrm{cm})$} \\
\hline & ${ }^{74} A s$ & ${ }^{84} R b$ & ${ }^{124} I$ \\
\hline titanium & 0.033 & 0.040 & 0.070 \\
\hline gold & 0.011 & 0.013 & 0.022 \\
\hline tungsten & 0.010 & 0.013 & 0.021 \\
\hline water & 0.106 & 0.129 & 0.227 \\
\hline
\end{tabular}

CSDA range is a very close approximation to the average path length traveled by a charged particle as it slows down to rest, calculated in the continuous-slowing-down approximation. In this approximation, the rate of energy loss at every point along the track is assumed to be equal to the same as the total stopping power. Energy-loss 
fluctuations are neglected. The CSDA range is obtained by integrating the reciprocal of the total stopping power with respect to energy.

It can be seen that even the fastest positron will be stopped with $0.022 \mathrm{~cm}$ of gold or $0.070 \mathrm{~cm}$ titanium which is the material used to make the capsule for PeTrack markers. The dose delivered to the patients from the marker depends on marker activity at the time of implantation and decreases rapidly with increasing distance. Dose to a small tissue volume surrounding the marker, from these isotopes, can be $\sim 70-500$ cGy to $4-12$ cc of tissue, in about 4 weeks, table 2.3 [1]. It should also be noted that, since these markers are implanted inside the tumor most of the dose from the markers is actually deposited into the tumor itself.

Table 2.3 Life time dose at different distances from the marker with different isotopes (activity=3.7 MBq) [1]. Reproduced by permission.

\begin{tabular}{ccccc}
\hline \hline & & \multicolumn{3}{c}{$\begin{array}{c}\text { Lifetime dose (Gy) at a different distance and the } \\
\text { affected volume }\end{array}$} \\
\cline { 3 - 5 } & $\begin{array}{c}\text { Half-life } \\
\text { Isotope }\end{array}$ & $\begin{array}{c}5 \mathrm{~mm} \\
\left(0.5 \mathrm{~cm}^{3}\right)\end{array}$ & $\begin{array}{c}10 \mathrm{~mm} \\
\left(4.2 \mathrm{~cm}^{3}\right)\end{array}$ & $\begin{array}{c}15 \mathrm{~mm} \\
\left(14 \mathrm{~cm}^{3}\right)\end{array}$ \\
\hline${ }^{124} \mathrm{I}$ & 4.2 & 2.6 & 0.7 & 0.32 \\
${ }^{74} \mathrm{As}$ & 17.8 & 9.0 & 2.46 & 1.09 \\
${ }^{84} \mathrm{Rb}$ & 32.7 & 18.6 & 4.96 & 2.24 \\
\hline \hline
\end{tabular}




\section{3- Detector module}

Two pairs of position-sensitive gamma detector modules for PeTrack will be installed on the linear accelerator gantry at 50-70 $\mathrm{cm}$ from the isocentre (Figure 2.1.1). The dimensions of the detector module are estimated to be $20 \times 20 \times 30 \mathrm{~cm}$. Detectors A1

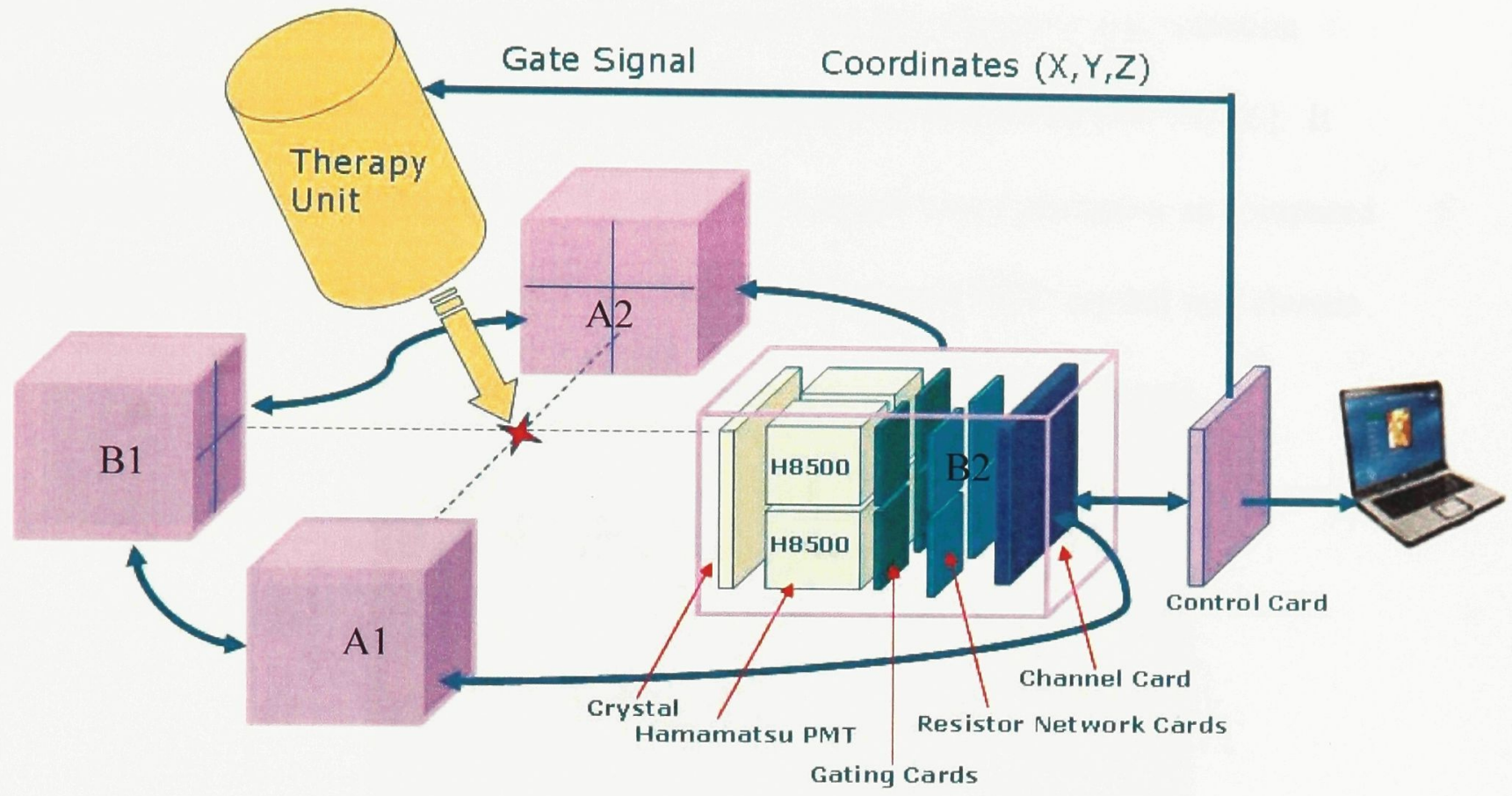

Figure 2.3.1 Schematic of the PeTrack Detector modules

and B1 will be attached to the Linac head. Detectors A2 and B2 will also be mounted on the Linac gantry through adjustable arms. The detector modules will be able to rotate around the patient with the gantry. By acquiring 60-200 positron annihilation events from each marker, the position of the marker can be determined with sub-millimeter accuracy. Each detector module composed of a $2 \times 2$ array of $5.2 \times 5.2 \mathrm{~cm}^{2}$ Bismuth Germinate (BGO) crystal blocks, a Hamamatsu position-sensitive photomultiplier immediately behind the crystal, front end electronics and gating circuit. Each detector module will have a field of view of $10.4 \times 10.4 \mathrm{~cm}^{2}$, which is sufficient to cover the range of 
respiratory motion.

Figure 2.3.1 is a schematic of the PeTrack detector modules.

\section{BGO crystal}

Bismuth Germinate crystal is known for its high detection efficiency, low afterglow and high radiation hardness. It was found that the afterglow and radiation hardness of BGO crystal differ depending on doping and manufacture $[24,25,26]$. It was reported that some undoped BGO crystal have a much lower afterglow as compared with doped BGO [27]. Based on these characteristics undoped BGO crystal was chosen

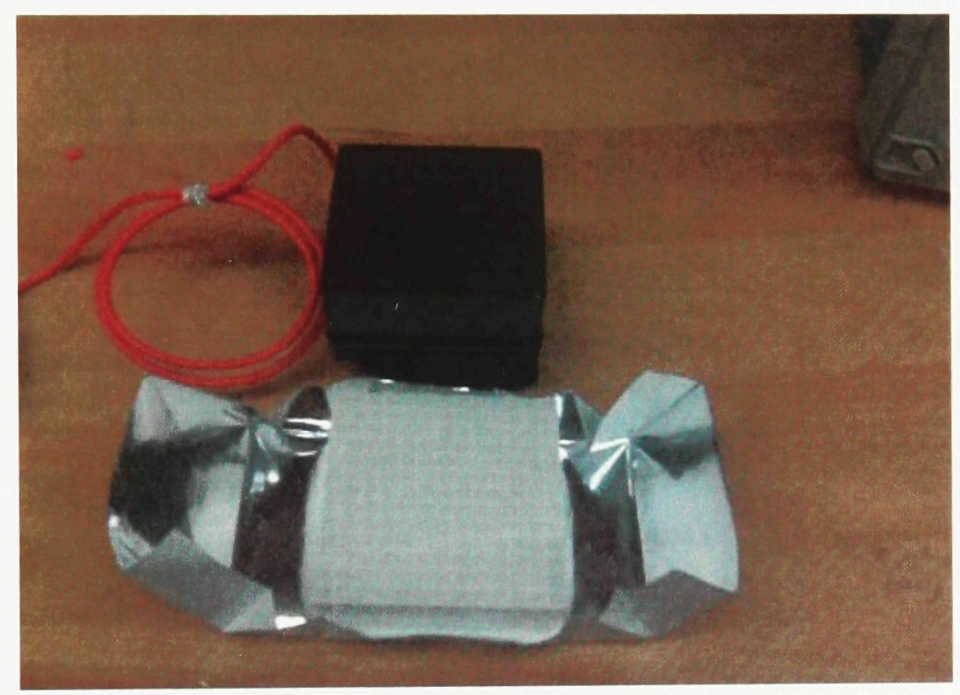

Figure 2.3.2 A $5.2 \times 5.2 \mathrm{~cm}^{2}$ BGO crystal pixelated to $13 \times 13$ arrays of $4 \times 4$ $\mathrm{mm}^{2}$ elements and an H8500 Hamamatsu photomultiplier

to be used for PeTrack detectors (Dr. Tong Xu, et. al. 2006). Each detector module has a $2 \times 2$ array of $5.2 \times 5.2 \mathrm{~cm}^{2}$ BGO crystal blocks and each block is pixelated to a $13 \times 13$ array of $4 \times 4 \mathrm{~mm}^{2}$ elements. Each crystal block is being readout by a position sensitive photomultiplier. Figure 2.3 .2 shows a $5.2 \times 5.2 \mathrm{~cm}^{2}$ crystal block and an H8500 Hamamatsu photomultiplier. 


\section{Position Sensitive Photomultiplier}

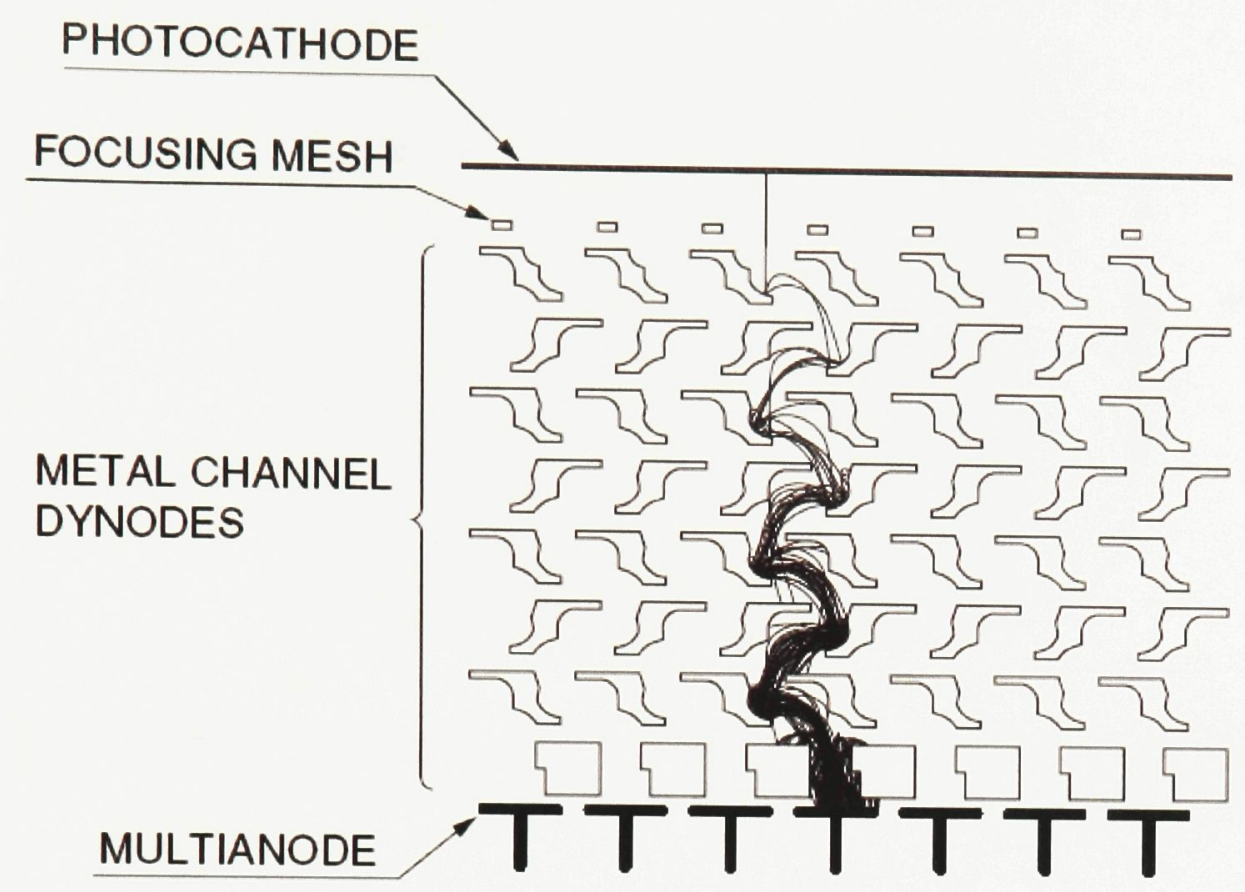

Figure 2.3.3 Electrode structure and electron trajectory of a PositionSensitive PMT [39].Reproduced with permission of Hamamatsu Co.

Position-sensitive photomultipliers consist of a metal channel dynode structure combined with multianode readout. Figure 2.3.3 shows the electrode structure for metal channel dynodes and the associated electron trajectories. The photoelectrons emitted from the photocathode are directed onto the first dynode by the focusing mesh and then flow to the second dynode, third dynode....last dynode and finally to the anode, while being multiplied with a minimum spatial spread in the secondary electron flow [39]. PMT provide a gain on the order of $10^{6}$ to $10^{7}$ of detected light signal by electron avalanches through a cathode - dynodes - anode system. High voltages are applied between dynodes to keep accelerating the secondary electrons and generate avalanches. 


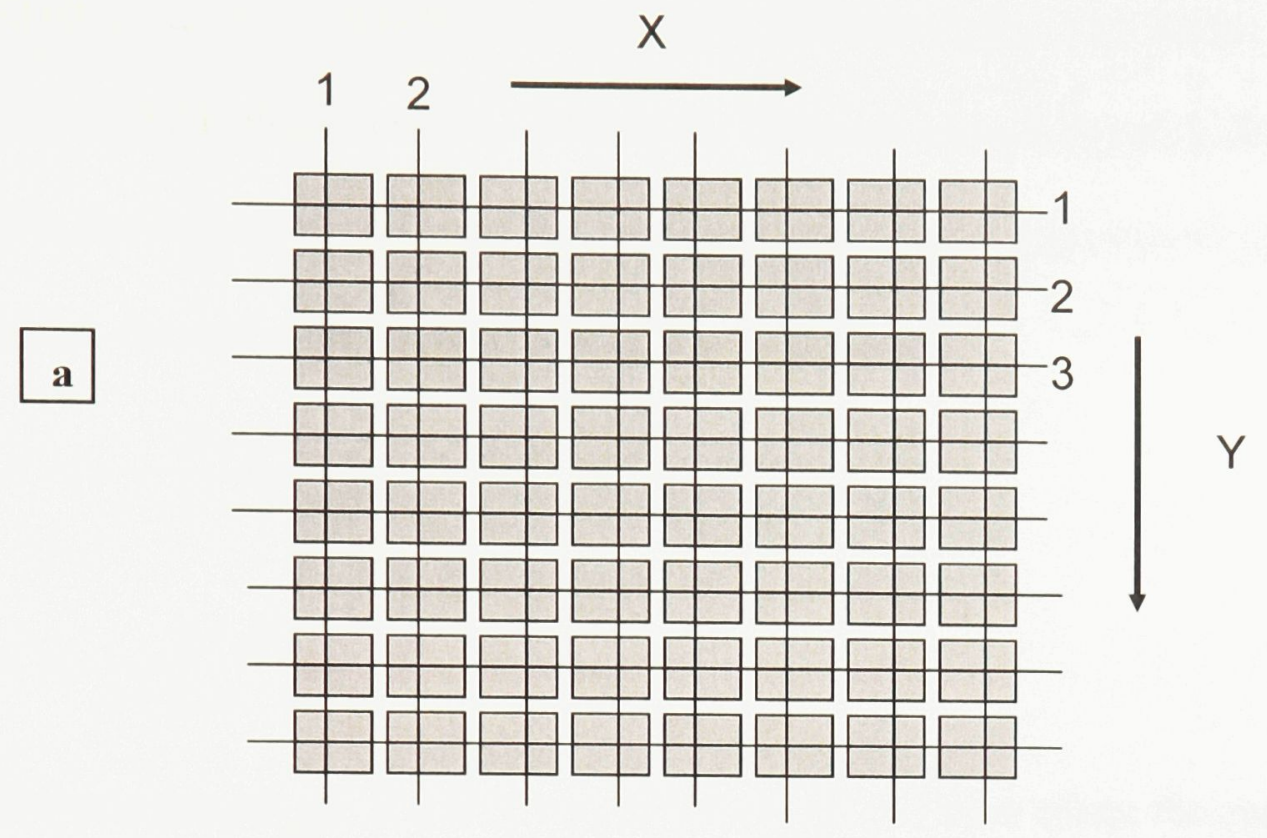

Figure 2.3.4 a: Anode pad array of PMT with the position coordinates $x, y$ b: Active resistive network and four output signals [39]

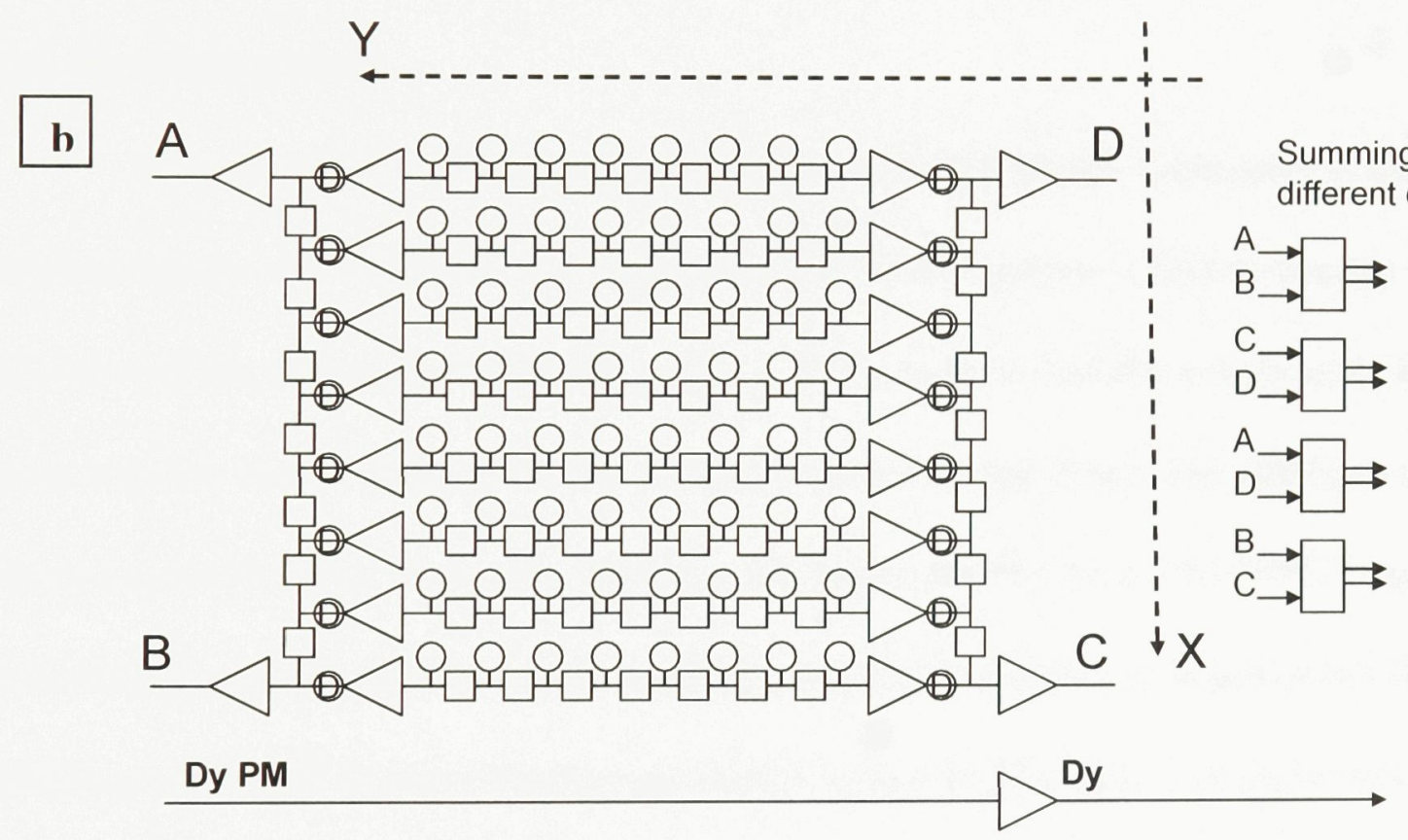

B corner marking, PM-HV cable

The position sensitive photomultiplier (H-8500 Hamamatsu Co.) has $8 \times 8$ anode pads of

$5.6 \mathrm{~mm} \times 5.6 \mathrm{~mm}$ size and $6 \mathrm{~mm}$ pitch. The 64 output signals from the anode pads are fed into an active resistor network. Four output signals are transformed from this network to the pulse processing board where the $\mathrm{x}$ and $\mathrm{y}$ coordinates of the absorption events are calculated [27]. Figure 2.3.4 shows a: the anode pad array of PMT with the position 
Coordinates $\mathrm{X}, \mathrm{Y}$, and $\mathrm{b}$ : the active resistive network and four output signals.

The active resistor network converts the 64 anode signals to four position signals. These four output signals A,D and B, C are transferred to the digital pulse processing board where the ratios for position $\mathrm{X}$ and $\mathrm{Y}$ are calculated.

$$
\begin{aligned}
& X \propto \frac{B+C}{A+D+B+C} \\
& Y \propto \frac{A+B}{A+B+C+D}
\end{aligned}
$$

These represent the $\mathrm{X}$ and $\mathrm{Y}$ coordinates of the position where the energy absorption event occurred. Total energy absorbed is proportional to the sum of the four signals, $\mathrm{A}+\mathrm{B}+\mathrm{C}+\mathrm{D}$.

\section{4- Readout system}

Mesytec MPET (mesytec GmbH \& Co. KG, Putzbrunn, Germany) is used as the readout system for PeTrack. The main function of this system is first to decode the X, Y position of the events, from signals A, B, C, D. Next is to find the coincidence events in a time window that is defined for the system ( $35 \mathrm{nsec}$ in our case). The software used is Mesydaq and it is the main user interface for the readout system. The MPET main allows connecting up to 8 position sensitive photo multipliers coupled to scintillators (BGO). PMTs are grouped in two coincidence groups with 4 PMTs each. For those two groups a fixed coincidence time window of $35 \mathrm{~ns}$ is implemented. Signal amplitudes, positions and coincidence time of the two responding PMTs are digitized and transmitted as 10 bit values [31]. An intelligence central processing device (MCPD2) is used for system control and buffering which transmits the data via Ethernet to a PC. The MCPD2 can serve up to 4 MPET- 8 modules. 
After system setup and calibration, we will have the X, Y coordinates of the energy absorption events and from these data we want to find the location of the markers implanted inside or at the site of the tumor. This will be done with a multi-marker tracking algorithm.

\section{5 -Single maker and multi-marker tracking algorithm}

\section{-Single marker algorithm}

Single marker algorithm is used in the positron emission particle tracking (PEPT) technique. For industrial applications, the PEPT tracks a single marker labeled with positron emission isotope that is introduced into a flow system [28, 29]. One pair of gamma detectors tracks the marker's location from the detection of two back-to-back annihilation gammas (511 keV). If there was no scatter and back-to-back gammas where exactly at a 180 degrees, two pairs of gammas whose coincidence line cross at the point of emission, can determine the location of the point source. It should also be noted that resolution of the detector is limited and therefore to triangulate the location of the marker additional gamma pairs are required. Single marker algorithm finds a point in 3D space that has the minimum distance to the $\mathrm{N}$ collected gamma coincidence lines and that would be the location of the marker. Therefore the localization accuracy is $L / \sqrt{ } N$, where

$\mathrm{L}$ is the intrinsic spatial resolution of the system and $\mathrm{N}$ is the number of detected coincidence lines. For example, in a PEPT system where intrinsic spatial resolution is 6 $\mathrm{mm}$, to bring the accuracy to $1 \mathrm{~mm}, 36$ coincidence lines should be collected, and to have an accurate real-time tracking of the positron emission marker, this is done in a fraction of a second. In order to track tumor's location and orientation using positron emission markers, at least three fiducial markers are required [1]. A multiple particle-tracking 
algorithm for industrial applications proposed by Gundogdu and Tarcan was able to track two positron emission particles with 20-30 mm resolution [30]. However, 2 markers are not enough and 20-30 mm resolution is not sufficient for tumor tracking.

\section{-PeTrack multimarket algorithm}

PeTrack's multi-marker tracking algorithm can track more than 3 markers with sub-millimeter precision.

The first step of the PeTrack algorithm is to sort hundreds of unlabeled coincidence lines arising from 3 markers into 3 clusters. After classifying all the coincidence lines into three clusters, inside each cluster the position of each marker is determined from its set of coincidence lines. Clustering the coincidence lines and finding and assigning the location of the marker inside each cluster are based on an ExpectationMaximization clustering algorithm [18].

The PeTrack algorithm is based on the following steps:

1) Initialization: Based on CT images taken from the patient after implantation of the 3 markers, the location of them is approximately known. The algorithm uses those approximate locations as the initial locations of the markers.

2) Clustering: the next step is to group the coincidence lines based on their distance from the three markers. Coincidence lines will be grouped into 3 clusters corresponding to three markers.

3) Updating the location of the markers: Now that all the coincidence lines are clustered, inside each cluster the algorithm finds a point that is closest to all the coincidence lines in that cluster and that point will be the new location of the marker. 
4) Iterative process: With these new markers' locations step 2 and 3 of the algorithm are repeated. This means all the coincidence lines are grouped into 3 clusters based on their distance to these 3 new markers' locations and then from these clusters new locations of the markers will be found and markers' location will be updated. This iterative process will be repeated until it converges and the output will be the final location of the 3 markers.

\section{6-summary}

In this chapter an overview of the PeTrack system was provided and design of the detector modules was explained. The readout system of PeTrack was introduced as well as a flow chart of the PeTrack algorithm.

As mentioned in section 2.2, BGO crystal is being used as the scintillating crystal for PeTrack detector modules. In the next chapter, characteristics of BGO crystal are discussed in more details and the results of afterglow evaluation and energy resolution of the $\mathrm{BGO}$ crystal sample are also provided. 


\section{Chapter 3- BGO crystal}

In this chapter the characteristics that made bismuth germinate $\left(\mathrm{Bi}_{4} \mathrm{Ge}_{3} \mathrm{O}_{12}\right)$ a good candidate for PeTrack detector modules as a scintillating crystal, are discussed and the results of our evaluation of a BGO sample is provided.

\subsection{Characteristics of BGO}

Despite a rather low light yield at room temperature (12\% of NaI-Tl ) BGO has come to be recognized as a crystal scintillator whose high density, short radiation length, high stopping power, low afterglow, mechanical stability and short decay time (300 ns) make it attractive for a wide range of applications in high energy physics, space science and medical imaging [33].

Table 3.1 compares some characteristics of a few major inorganic scintillators.

Table 3.1 Comparison of major inorganic scintillators [34]

\begin{tabular}{|c|c|c|c|c|}
\hline Scintillator & NaI:Tl & CsI:TI & GSO & BGO \\
\hline Effective atomic number & 50 & 54 & 59 & 74 \\
\hline Density $\left(\mathrm{g} / \mathrm{cm}^{\underline{3}}\right)$ & 3.67 & 4.51 & 6.71 & 7.13 \\
\hline Peak wavelength $(\mathrm{nm})$ & 410 & 565 & 430 & 480 \\
\hline Light yield (relative) & 100 & 45 & 20 & 12 \\
\hline Scintillation decay time (ns) & 230 & 1000 & 60 & 300 \\
\hline Attenuation coefficient $\left(511 \mathrm{keV:}: \underline{\mathrm{cm}}^{-1}\right)$ & 0.34 & 0.41 & 0.674 & 0.955 \\
\hline
\end{tabular}

For $511 \mathrm{keV}$ gammas, $\mathrm{BGO}$ with highest atomic number and density among others has the highest attenuation coefficient (Table 3.1). The peak wavelength of BGO scintillation light is around $480 \mathrm{~nm}$.

\subsection{Radiation Hardness}

Since the scattered $\mathrm{x}$-ray field from a linear accelerator is very intense, radiation hardness of the scintillating crystal is a concern in the design of the PeTrack detectors. Radiation damage to the crystal happens when the electrons that are excited by radiation 
are trapped in oxygen vacancies and form the color centers in the crystal.

It has been reported that BGO crystal will show a reduction in light output after 1-10 krad of radiation $[24,27]$. It has also been observed that BGO crystal has the ability to self recover after irradiation [27]. The radiation background from a linear accelerator (Elekta Precise) was measured experimentally at the location of the PeTrack detector A1 and B1,

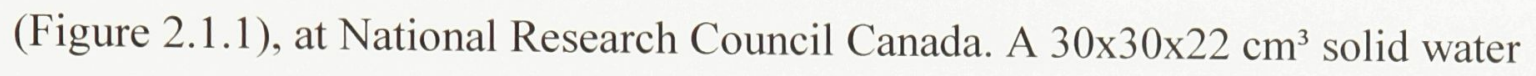
phantom was used to simulate the patient's scatter. The source to surface distance was $100 \mathrm{~cm}$ and source to isocentre was $110 \mathrm{~cm}$. The field size was chosen to be $10 \times 10 \mathrm{~cm}^{2}$ and 500 monitor units were delivered at $500 \mathrm{MU} / \mathrm{min}$ rate. An ion chamber, Exradin A19 Farmer-type chamber, was used. The build-up cap was about $7 \mathrm{~cm}$ diameter PMMA, and the calibration coefficient was $4.8 \mathrm{cGy}$ per $\mathrm{nC}$. The radiation background was measured for $6 \mathrm{MV}$ and $10 \mathrm{MV}$ beams. The results are listed in Table 3.2.

Table 3.2 Dose measurements in air with an ion chamber for different settings of the Linac and different locations of the PeTrack detector

\begin{tabular}{|l|l|l|}
\hline & $10 \mathrm{MV}, 200 \mathrm{~Hz}$ & $6 \mathrm{MV}, 400 \mathrm{~Hz}$ \\
\hline Forward position & $0.99 \mathrm{cGy}$ & $0.99 \mathrm{cGy}$ \\
\hline Back position & $0.43 \mathrm{cGy}$ & $0.46 \mathrm{cGy}$ \\
\hline
\end{tabular}

Back position means attached to the Linac head (A1 and B1 in figure 2.1.1) Forward means behind the phantom (A2 and B2 in figure 2.1.1)

It was found that at forward position, $\mathrm{A} 2$ and $\mathrm{B} 2$ in figure 2.1.1, we have a higher dose rate of about 0.99 rad per $500 \mathrm{MU}$ delivered. Assuming $1000 \mathrm{MU}$ delivered per patient and 35 patients a day in a normal clinical setup, the BGO crystal will accumulate about $70 \mathrm{rad}$ in one day. This is far lower than the 1-10 krad radiation hardness of BGO crystal 
[27], and as mentioned before since BGO has the ability to self recover after radiation [27], BGO crystal may fully recover overnight.

\subsection{Afterglow}

Afterglow is commonly understood as a very slow light emission from a scintillation crystal after the cessation of an excitation. Compared to the decay time of a scintillation crystal, a few hundred nanoseconds, afterglow can be as long as a few milliseconds.

It has been reported that BGO crystals has a very low afterglow: ranging from $0.005 \%$ at $0.2 \mathrm{~ms}$ to $0.005 \%$ at $3 \mathrm{~ms}$ [27]. Studies have shown that compared to doped BGO, pure BGO does not have a visible afterglow [27].

\section{4- Evaluation of BGO sample}

Since afterglow depends on the purity of the BGO crystal, the evaluation of afterglow for our BGO sample was required. The first evaluation was done with pulsed UV light. The crystal shown in figure 3.4 .1 is an undoped BGO crystal $\left(2 \times 2 \times 2 \mathrm{~cm}^{3}\right)$ that was examined for afterglow.

A Hamamatsu R980 PMT was coupled to the crystal with silicon grease. A UV filter was

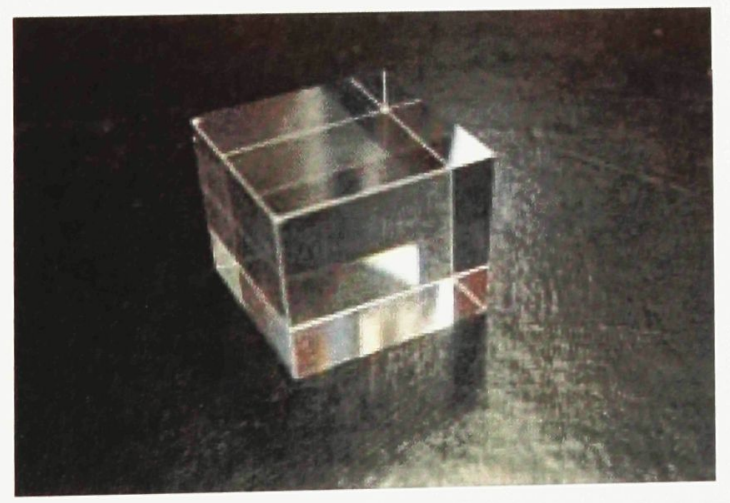

Figure 3.4.1 A $2 \times 2 \times 2 \mathrm{~cm}^{3}$ undoped BGO crystal. 
placed between crystal and PMT to prevent PMT being excited directly by intense UV light, i.e. only the scintillation light from the BGO crystal can pass the filter. Figure 3.4.2 shows the detector unit and a schematic of the electronics for primary evaluations.

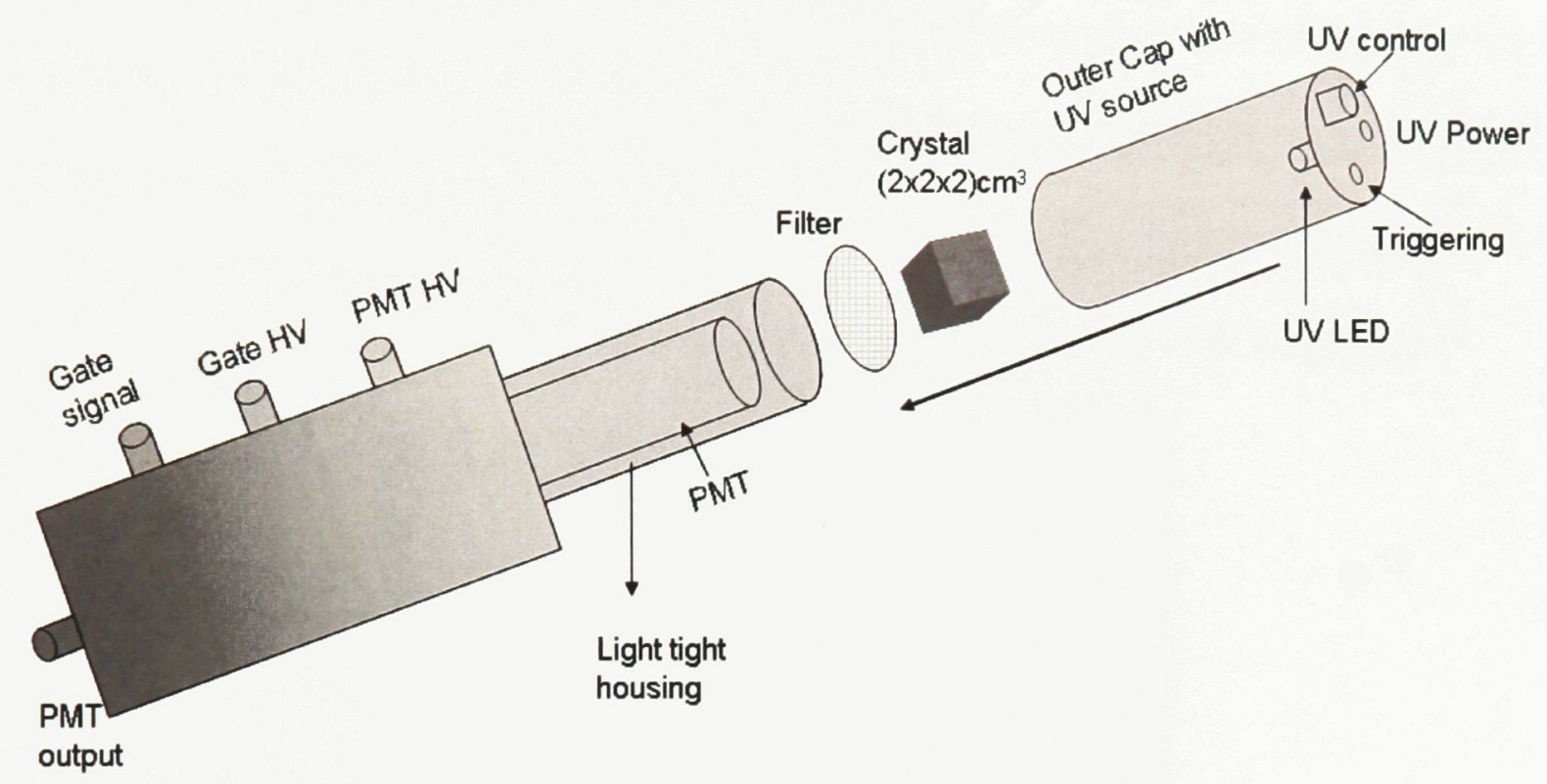

(a)

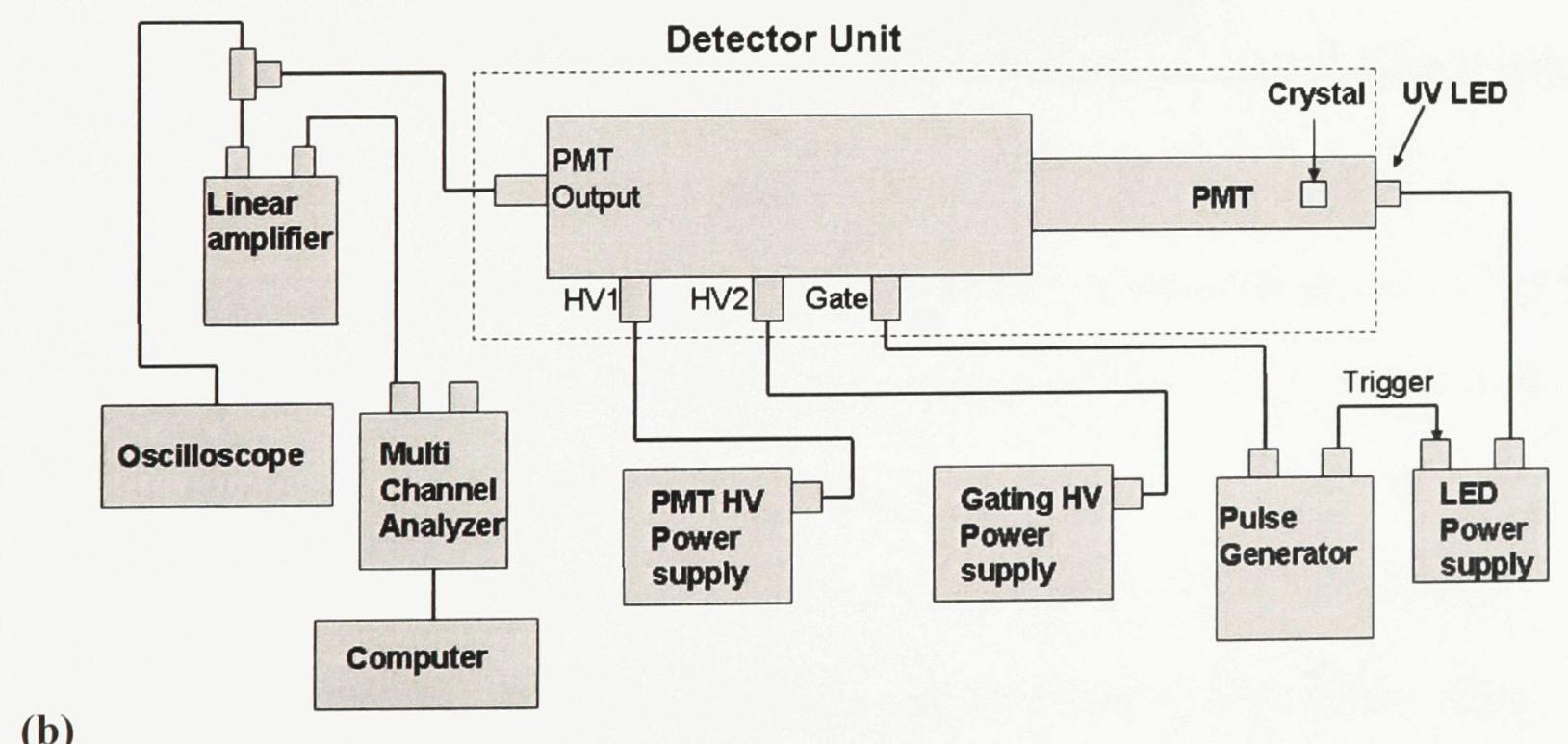

Figure 3.4.2 Detector unit (a), electronics schematic (b), used for primary evaluations. Crystal is coupled to the PMT with silicon grease, outer cap slides and covers the housing. 


\section{- Evaluating BGO afterglow from UV excitation}

An ultra violet LED with $305 \mathrm{~nm}$ wavelength was used to simulate the intense stimulation from scattered x-ray from Linac. The radiation dose to the crystal due to scattered $\mathrm{x}$ rays was measured to estimate the required excitation energy from LED. Scattered $\mathrm{x}$ rays from the Linac were measured for $500 \mathrm{MU}$ with a rate of $500 \mathrm{MU} / \mathrm{min}$. For a $6 \mathrm{MV}$ beam, the dose at $50 \mathrm{~cm}$ from isocentre (location of the PeTrack detectors) was measured to be $0.99 \mathrm{rad} / \mathrm{min}$. At this rate the total energy deposited to the crystal (57 g) is $0.6 \times 10^{-3} \mathrm{~J}$ per minute. Taking into account the frequency of the $\mathrm{x}$-ray pulse, 400 $\mathrm{Hz}$, and the pulse width of $10 \mu \mathrm{s}$, the power of the scattered $\mathrm{x}$ rays will be $0.15 \mathrm{~mW}$.

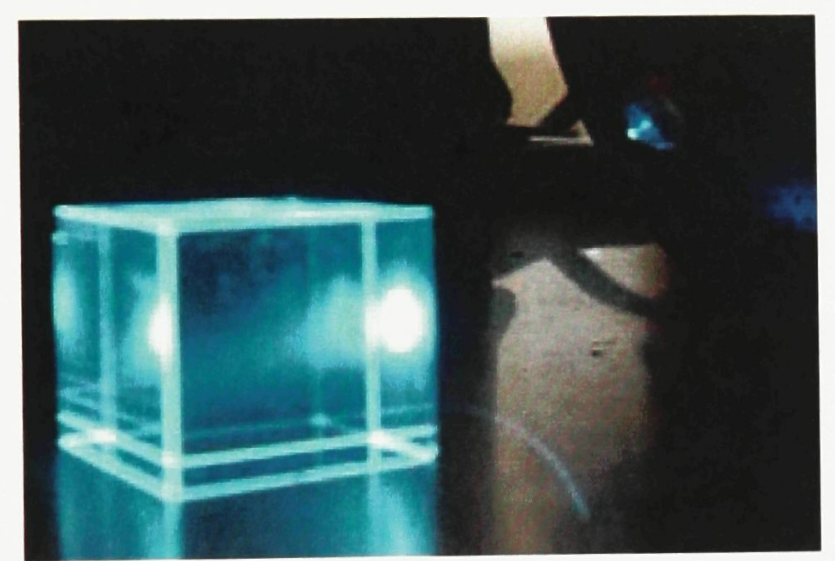

Figure 3.4.3 Showing the intensity of the UV light shining on a $2 \times 2 \times 2 \mathrm{~cm}^{3} B G O$ crystal The power of the UV LED can be as high as $2.4 \mathrm{~mW}$ at $180 \mathrm{~mA}$ (in pulse mode). Therefore, it is capable of simulating the crystal stimulation from scattered $\mathrm{x}$ rays. Figure 3.4.3 shows the intensity of the UV LED by shining it on the BGO crystal. The blue light is the scintillation light from $\mathrm{BGO}$ due to UV excitation.

Figure 3.4.2 (b) shows electronics schematic of the detector unit. During the afterglow experiment, the PMT was connected to a high voltage of 500 volts (the voltage of the PMT was set lower than the working voltage of the PMT ( $800 \mathrm{~V}$ to $1000 \mathrm{~V})$ to protect the PMT from possible damage from the intense UV light) and PMT output was measured 
and recorded with UV excitation of the BGO crystal. The UV was working in a pulse mode, with the pulse width of $40 \mu$ s.

\section{-Evaluating energy resolution}

The energy spectrum of a positron emission source ${ }^{22} \mathrm{Na}$ was obtained with a multi channel analyzer to measure the energy resolution of the BGO sample. The isotopes mentioned in section 2.2 are suitable for the PeTrack technique in clinical applications because their short half lives are comparable to the duration of the radiation therapy. In the lab, although we could work with any of those positron emission isotopes mentioned before, it was preferred to use an isotope that had a longer half life. Since the project was over a two year period, ${ }^{22} \mathrm{Na}$ with half life of 2.6 years was purchased and used during our research as our positron emission source. During the energy spectrum measurement, the PMT was supplied with normal working voltage of $830 \mathrm{~V}$.

\section{-Evaluating BGO respond from scatter Linac $x-$ ray}

The same setup (Figure 3.4.2), was also taken to the National Research Council of Canada for evaluation in a radiation environment of a Linac. At $6 \mathrm{MV}$ and $50 \mathrm{~Hz}$ setting, The PMT was connected to a high voltage of 830 volts and for different field sizes the PMT output was recorded with a Tektronix oscilloscope (UV filter was removed and crystal was directly coupled to the PMT with silicon grease).

\section{5- Results}

Figure 3.5.1 shows the UV signal and also the PMT output. It was observed that almost immediately after the UV signal was cut off, the PMT output disappeared, proving that there was not a visible afterglow in our BGO sample (PMT output signal from $511 \mathrm{keV}$ gamma is around $5 \mathrm{mV}$ and afterglow is around $0.2 \%$ of this signal which is negligible). 


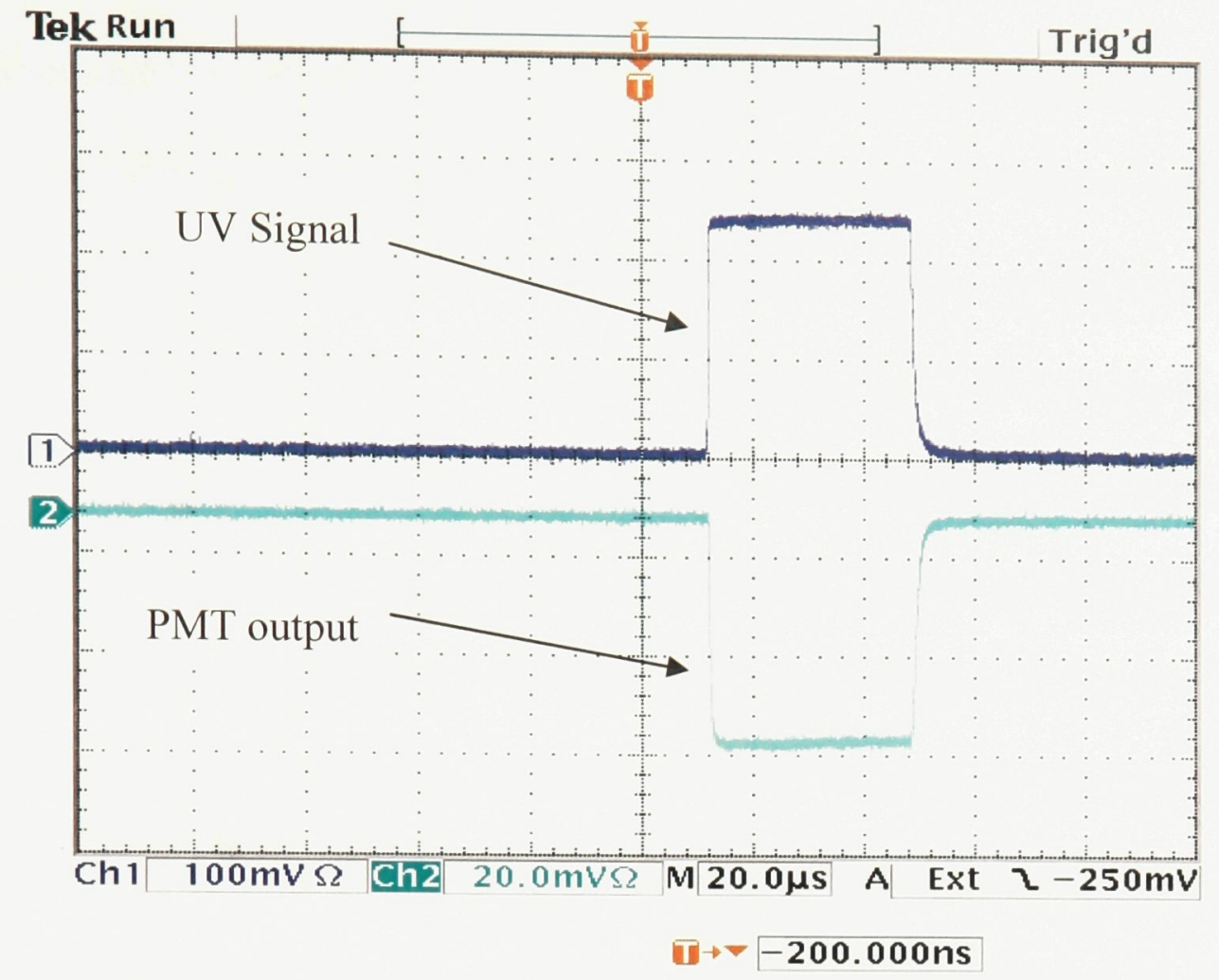

Figure 3.5.1 UV signal, top, and PMT output at 500V, bottom

Figure 3.5.2 shows the spectrum and it can be seen that both gamma peaks, $511 \mathrm{keV}$ and 1.21 MeV, are easily distinguished for the background. After fitting the $511 \mathrm{keV}$ peak with a Gaussian function the energy resolution (full with half maximum) was calculated to be $16 \%$. This energy resolution is well within the normal requirement of a PET system as well as PeTrack design parameters (energy resolution of better than 25\%). When the

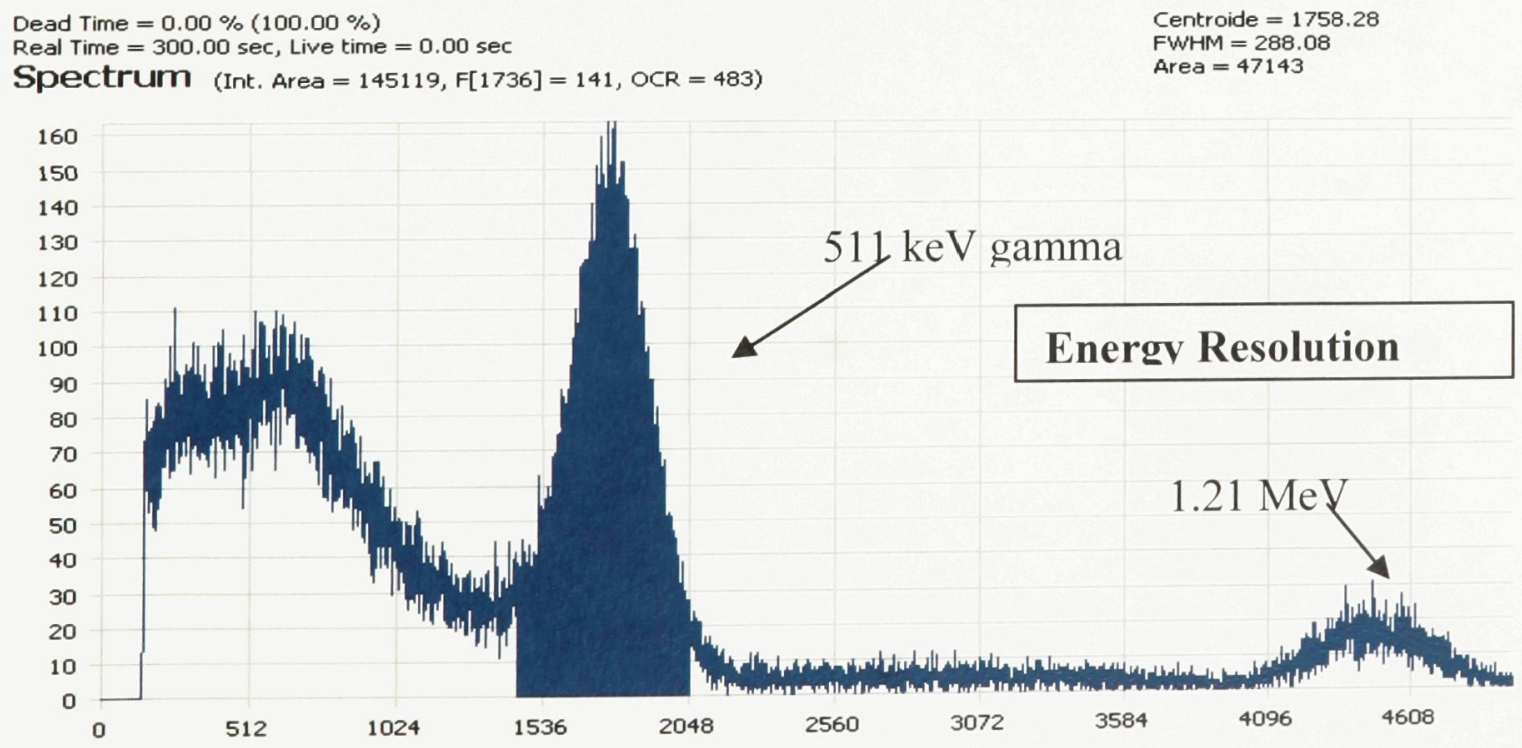

Figure 3.5.2 Energy resolution of BGO for $511 \mathrm{keV}$ gamma rays, high voltage 830 
detector assembly was put into the radiation environment of Linac machine, a very intense scatter signal was observed. Figure 3.5.3 shows PMT output signal amplitude for different field sizes. Once more from these results, we could see that there is no visible afterglow from BGO crystal. But another interesting observation was also made from figure 3.5.3. In this picture, it can be seen that with changing the field size, i.e. scatter level, the PMT output changes a little. For example, the scatter signal with zero field size should be about $1 / 10$ of that of $15 \times 15 \mathrm{~cm}^{2}$ field (see section 4.3, figure 4.3.13). However, they have the same amplitude as shown in Figure 3.5.3. This is an indication of PMT being saturated due to the intense scatter radiation from the Linac. This justifies the use of a gating circuit to protect the PMT from saturation, and prevent temporary overload or even permanent damage to the dynode system [36, 39].

In the next chapter, the principle of PMT gating is discussed, and design and evaluation of the gating is presented.

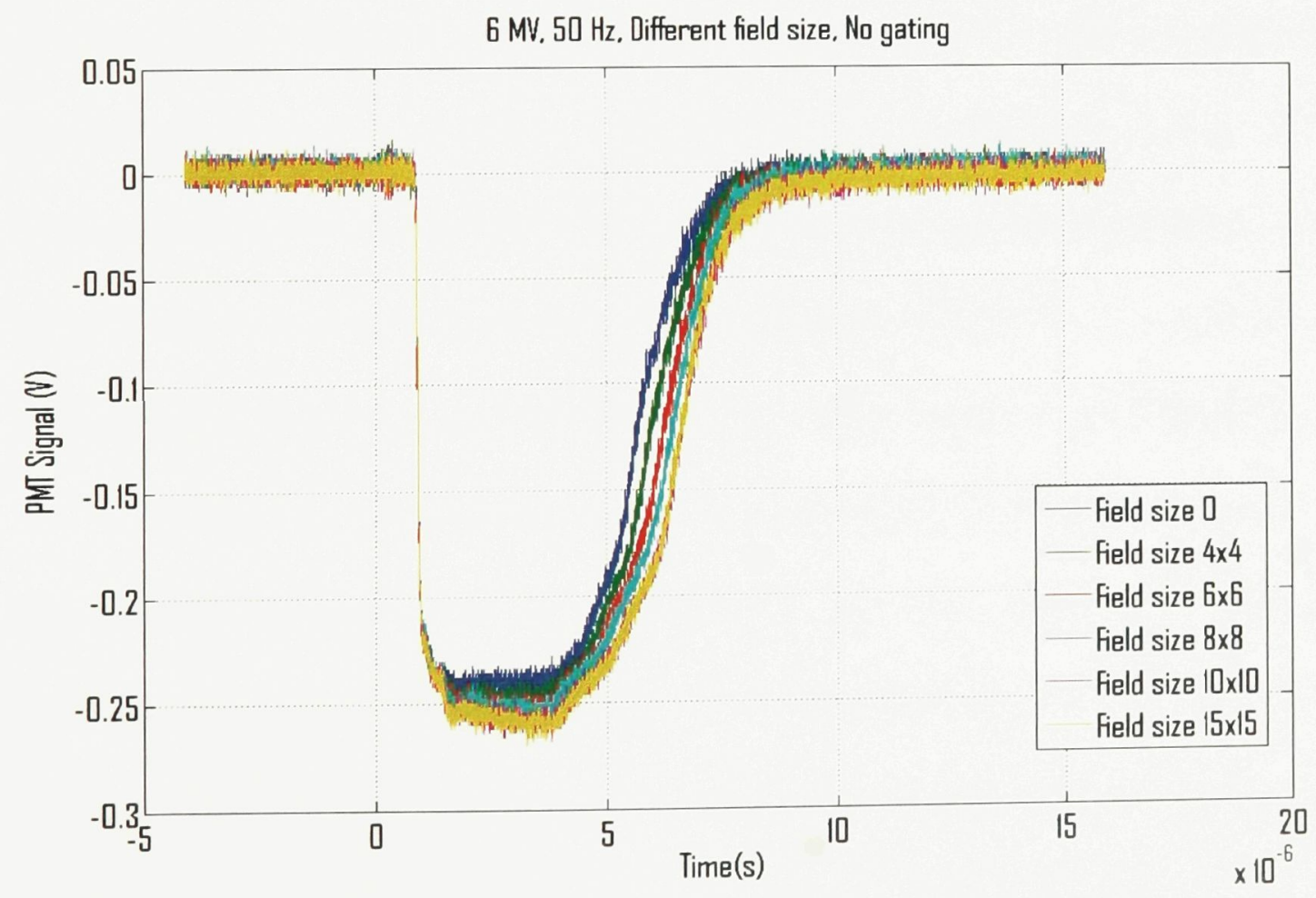

Figure 3.5.3 PMT output due to scatter x-ray from a Linac at different field sizes 


\section{Chapter 4- Gating of PeTrack detector}

As mentioned in the previous chapter, scattered x-ray field in the environment of a Linac is so intense that it can cause temporary overload or even permanent damage to the PMT and the electronics. It is best to gate the PMT off during the Linac signal. In this chapter, the principles of gating, as well as design and evaluation of the gating circuit used in the PeTrack system, are presented.

\section{1- Principles of gating}

A Linac works in pulse mode with a pulse rate of $25-400 \mathrm{~Hz}$ and a pulse width of only a few microseconds. The time intervals between the pulses are 2.5 to $40 \mathrm{~ms}$. The scattered x-ray field from the NRC Linac was measured at $500 \mathrm{MU}$ with a rate of 500 $\mathrm{MU} / \mathrm{min}$, for $6 \mathrm{MV}$ beam, and the dose at $50 \mathrm{~cm}$ from isocentre (location of the PeTrack detectors) was measured to be $0.99 \mathrm{rad} / \mathrm{min}$. The total energy deposited to the crystal (57 $\mathrm{g}$ ), is $0.6 \times 10^{-3} \mathrm{~J}$ per minute. Taking into account the frequency of the $\mathrm{x}$-ray pulse, 400 $\mathrm{Hz}$, the energy deposited to the crystal per pulse is $2.5 \times 10^{-8} \mathrm{~J}$ which is much higher than the energy of one gamma ray from positron annihilation $\left(511 \mathrm{keV}\right.$ or $\left.8.2 \times 10^{-14} \mathrm{~J}\right)$. Therefore the PeTrack detectors should be gated to not acquire data during the x-ray pulse. This is achieved by gating the photomultiplier tube (PMT), i.e. turn the PMT off during the intense x-ray pulse and turn it back on during the time between the pulses.

Gating of a photomultiplier is a common technique when it is required to observe a small signal compare to an intense excitation. As shown in section 2.3, the PMT provide a gain in the order of $10^{6}$ to $10^{7}$ of detected light signal by electron avalanches through a cathode - dynodes - anode system. High voltages are applied between dynodes to keep accelerating the secondary electrons and generate avalanches. When there is an 
intense signal, in our case scattered $\mathrm{x}$ rays, it is desirable to gate the photomultiplier off for a certain period of time in order to prevent the dynode system from suffering temporary overload, and even permanent damage. As shown in Chapter 3, figure 3.5.3, the PMT was saturated by the scattered x-ray pulses; which will cause significant stress on the PMT and the front end electronics. Particular gating systems tend to relate to a particular type of photomultiplier. Some of the gating techniques available are as follows:

1. Pulsing overall high voltage supply

2. Pulsing group of dynodes

3. Pulsing the photocathode

4. Pulsing the first dynode

5. Pulsing a focusing or gating electrode, if present Among these techniques pulsing groups of dynodes has been reported to be the most

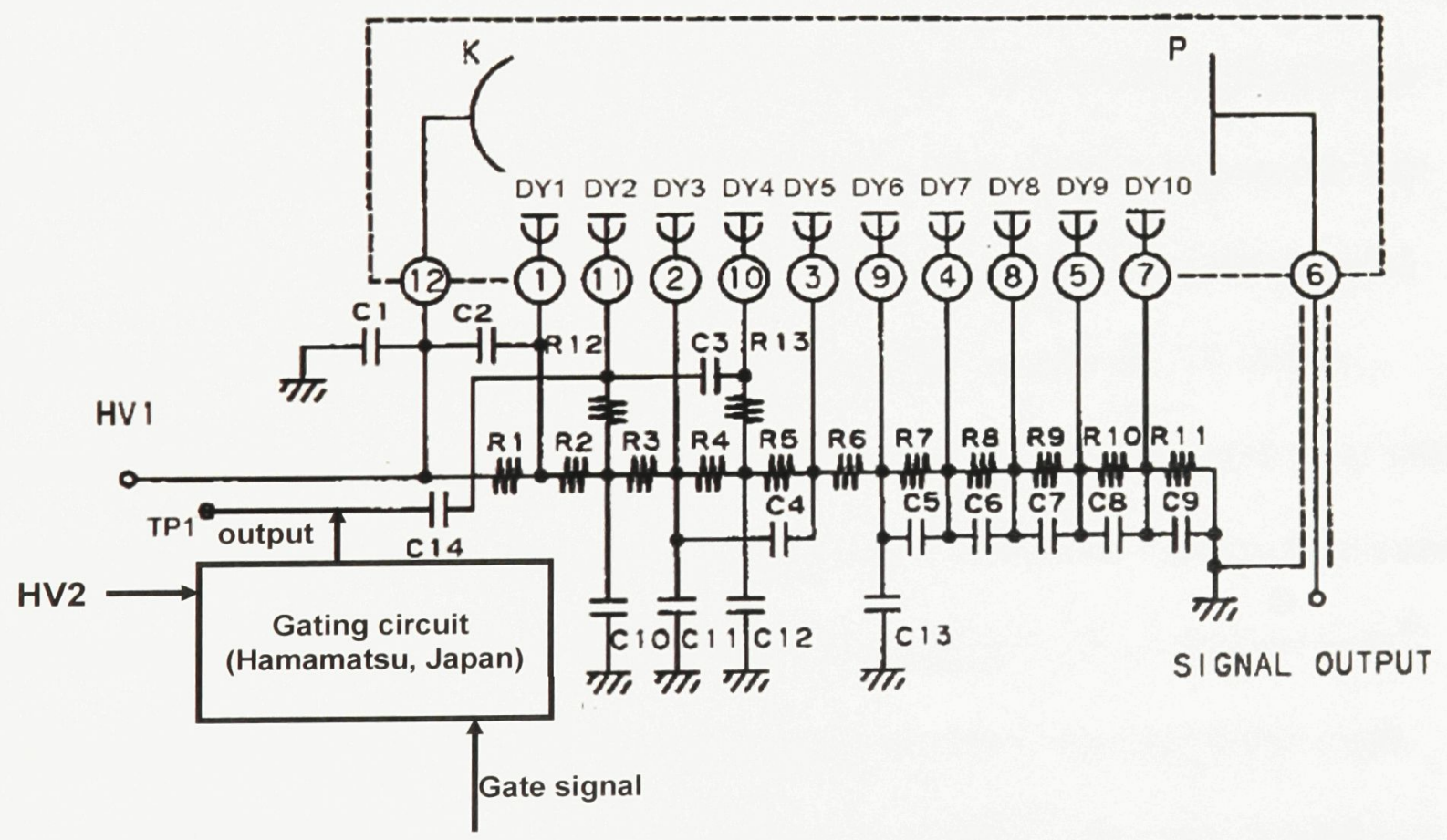

Figure 4.1.1 Schematics of gating circuit. $\mathrm{K}$ is the photocathode. DY1...DY10 are the PMT the dynodes $1 \ldots 10 . \mathrm{P}$ is the anode. HV1 is the high voltage power supply for the PMT. HV2 is the gating voltage. GATE is the input for gating pulse. Reproduced with permission of Hamamtsu Co, Japan 
successful technique [36]. This technique has been used in our design and we are pulsing dynodes number 2 (DY2) and 4 (DY4). As a rough approximation, reversing the potential between adjacent dynodes such that secondaries are repelled by succeeding dynodes rather than accelerated will reduce the output signal by 30 to 100 times.

Using another such a reverse biased stage in the multiplier (not including DY1), can possibly attain a cutoff ratio of $10^{4}$ [36].

Figure 4.1.1 shows the design of the gating circuit. The design was adopted from a PMT gating circuit designed by Hamamatsu. The original circuit was designed for a gate width of 10-100 ns. The circuit was adjusted to work with gate width of 10-500 $\mu \mathrm{s}$.

The PMT is connected to a high negative voltage (HV1) of -1000 volt. The gating voltage (HV2) is $-250 \mathrm{~V}$. Before applying the gating signal (GATE), point TP1 (Test Point one)which is connected to one side of the large capacitor $\mathrm{C} 14,1000 \mathrm{pF}$, is sitting at a negative voltage of -250 volt, which is equal to HV2. The other side of C14 is connected to dynode 2 (DY2) which is sitting at -750 volt. Therefore C14 has 500 volts across it (time constant $2.5 \mathrm{~ms}$ ). The other large capacitor, $\mathrm{C} 3,10 \mathrm{nF}$, has -750 volt on one side and -583 volt on the other side, resulting in 167 volts across. When the gating signal (GATE), of -4 volt is applied, TP1 which is connected to one side of C14, will be connected to ground. Since C14 is a large capacitor and just before this switch was sitting at 500 volts across, it will try to hold the same voltage across (time constant $30 \mathrm{~ms}$ ) and this will bring the voltage of dynode 2 (DY2), which originally was $-750 \mathrm{~V}$, to $-500 \mathrm{~V}$. This means that now dynode 3 (DY3), that is sitting on $-666 \mathrm{~V}$ is more negative than dynode 2 (DY2) and electrons will not be accelerated toward it. The same procedure is happening for dynodes 4 and 5. When the gating voltage is applied and transistors Q1 and 
Q2 switch to off and on respectively, one side of C3 will be connected to - $500 \mathrm{~V}$ and since C3 had $167 \mathrm{~V}$ across, it will try to keep this voltage across and as a result it will bring the voltage of the dynode 4 to -416 which is more positive than the voltage of dynode 5 which is -500 and therefore electrons will not accelerate from dynode 4 toward dynode 5. These reverse biased stages will force the PMT to be gated off for the duration of gating signal $(10 \mu \mathrm{s})$. Figure 4.1.2 shows the gating signal and also the voltage

a

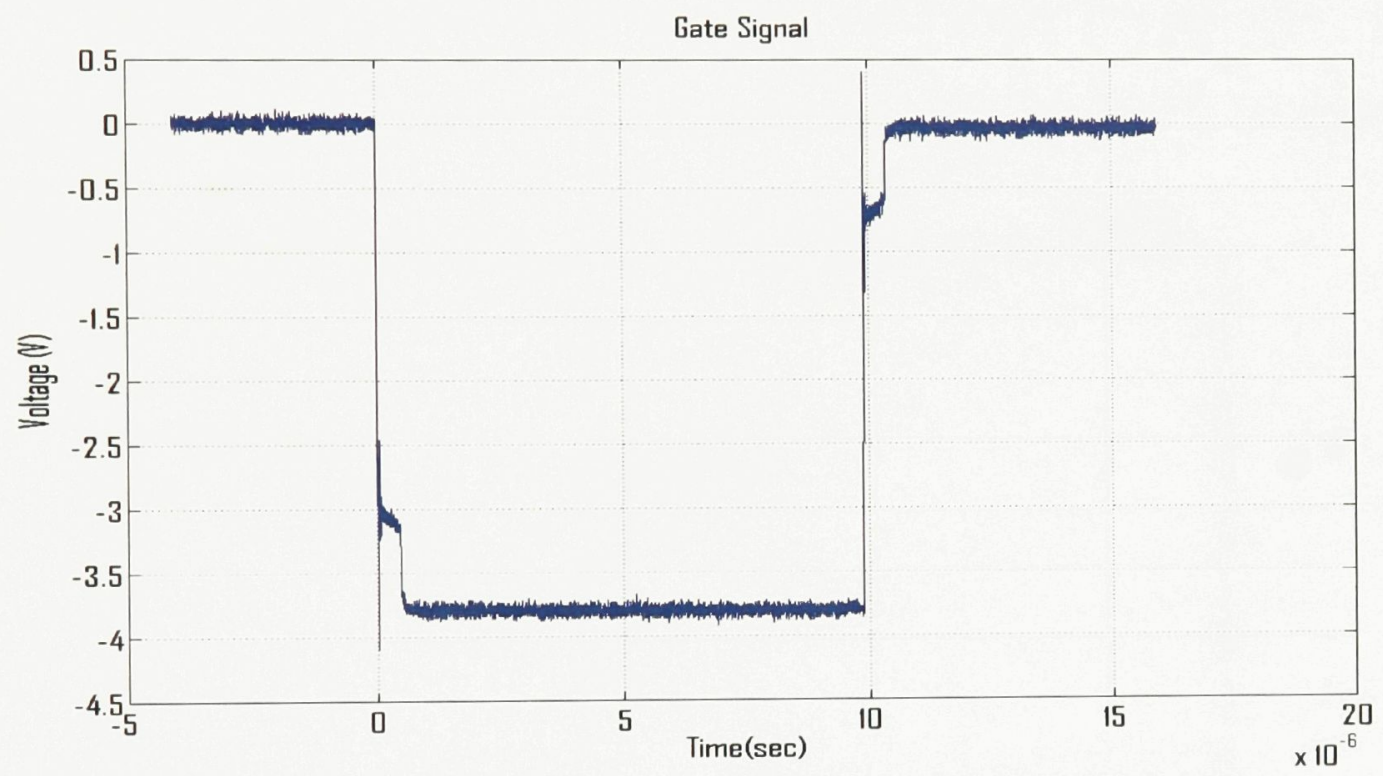

$\mathrm{b}$

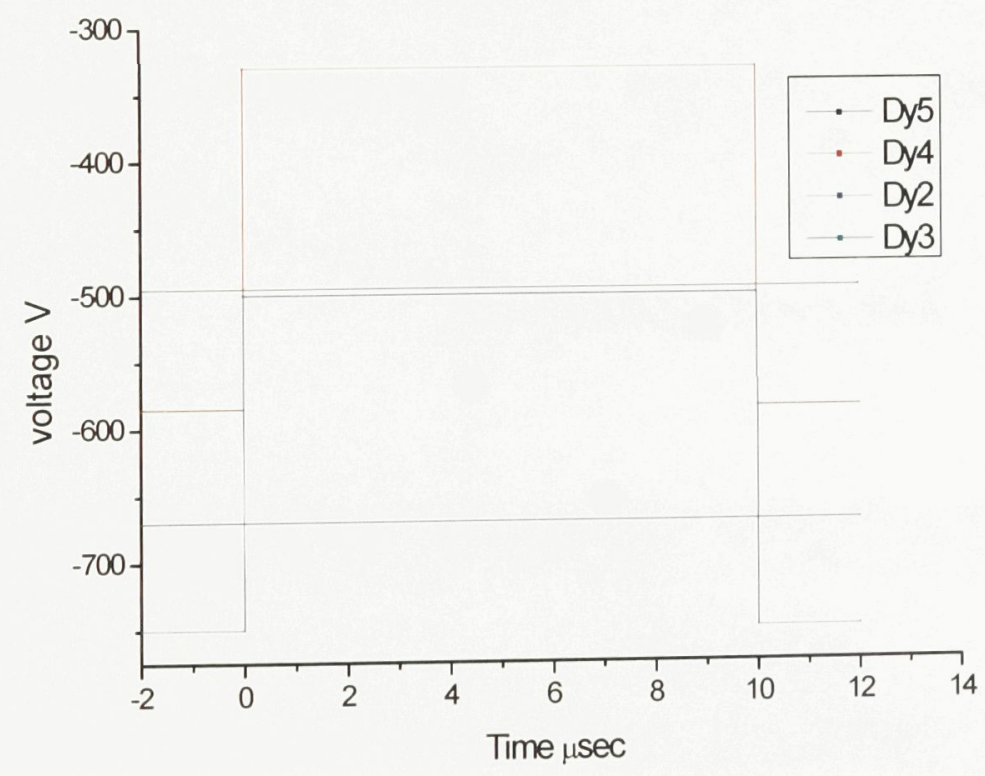

Figure 4.1.2 a: Gate signal, $10 \mu$ s, b: Dynode 2, 3, 4, and 5 voltages before, during and after gate signal. 
changes for DY2, DY3, DY4, and DY5 before, during and after the gating signal.

To achieve a highest ratio of PMT shutoff, the gating voltage was designed to be about $1 / 4$ of the PMT voltage, i.e. HV2 $=1 / 4 \mathrm{HV} 1$.

The gating voltage HV2 should be negative. However, the gating voltage value will be quoted by its absolute value in the rest of the report for the sake of simplicity.

\section{2- Evaluation of the gating circuit with UV excitation}

Evaluation of the gating circuit was done in two stages. The first stage was done in our own lab using an intense ultraviolet LED $(1.8 \mathrm{~mW})$. The setup is the same as

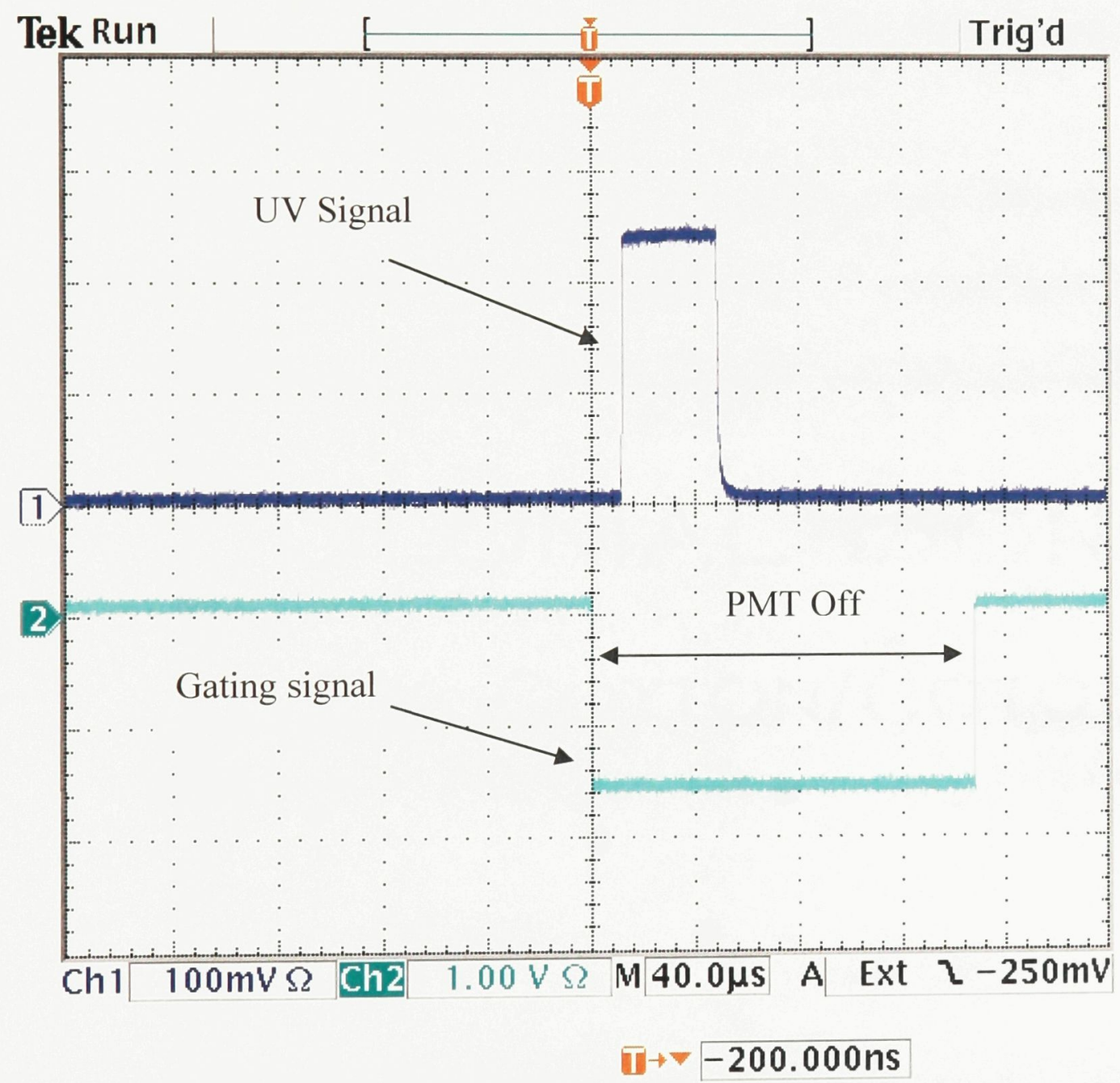

Figure 4.2.1 UV LED signal (top) and gating signal (bottom)

shown in figure 3.4.2. The UV source was set to work in a pulse mode with pulse width 
of $40 \mu$ s (Figure 4.2.1). The width of the gating signal was chosen to be $250 \mu \mathrm{s}$ (much longer than the UV pulse width to remove afterglow of crystal if there is any). A $2 \times 2 \times 2$ $\mathrm{cm}^{3}$ BGO crystal was used as our scintillating crystal. The crystal was coupled to an Hamamatsu PMT R980 with silicon grease (a UV filter was also placed in front of the PMT to prevent the direct excitation of the PMT with intense UV light). The PMT was then attached to our gating circuit and the output signal of the PMT was read on a Tektronix TDS 3012B oscilloscope. The PMT was connected to the high voltage supply and the voltage was set to 500 volts (the voltage of the PMT was set lower than the working voltage of the PMT, $800 \mathrm{~V}$ to $1000 \mathrm{~V}$, to protect the PMT from possible damage from the intense UV light).

Before connecting the gating signal and applying the gating voltage, the output signal on the PMT was recorded and is shown in figure 4.2.2. The output signal was around $50 \mathrm{mV}$.

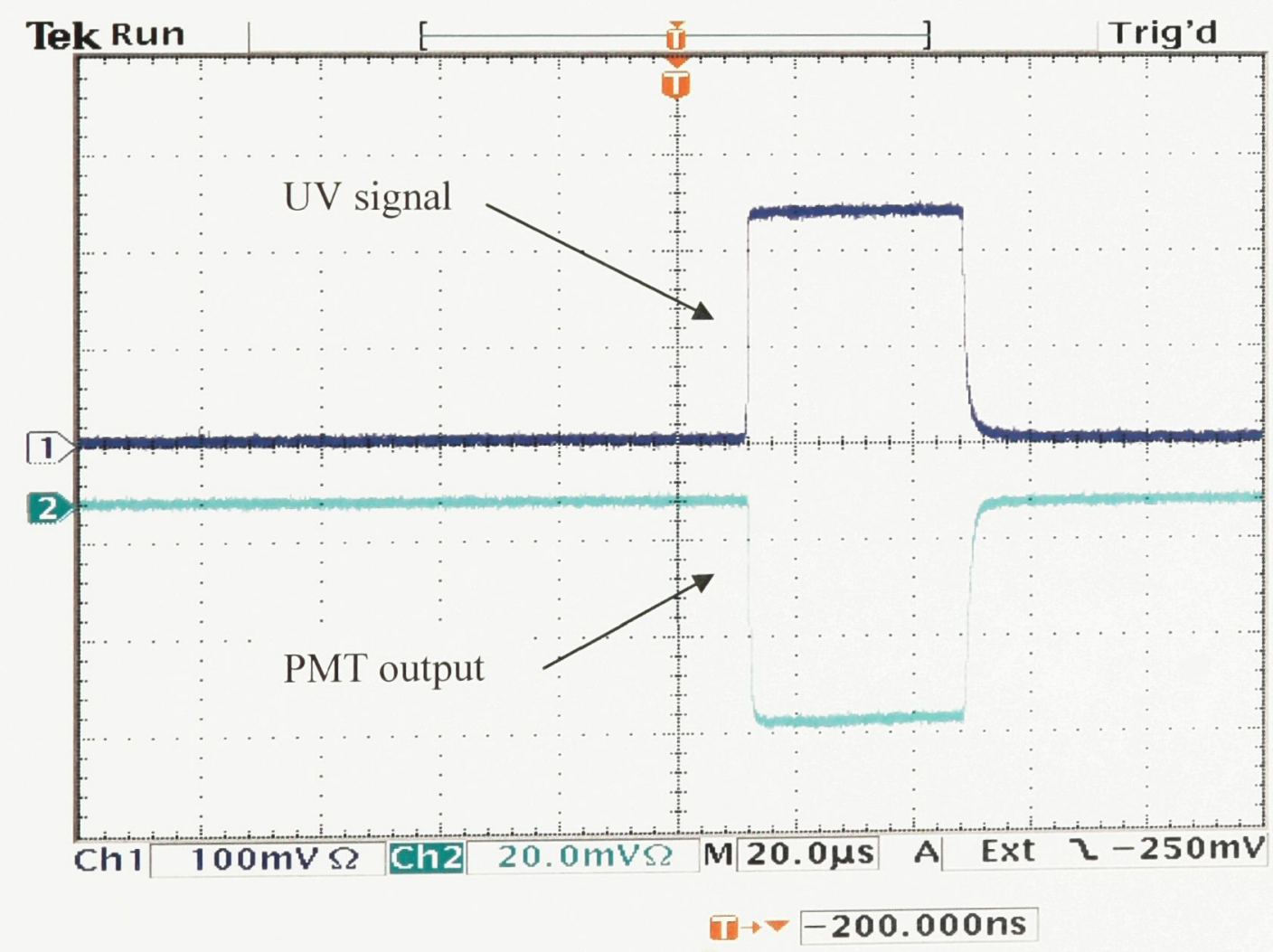

Figure 4.2.2 UV signal and PMT output without gating 


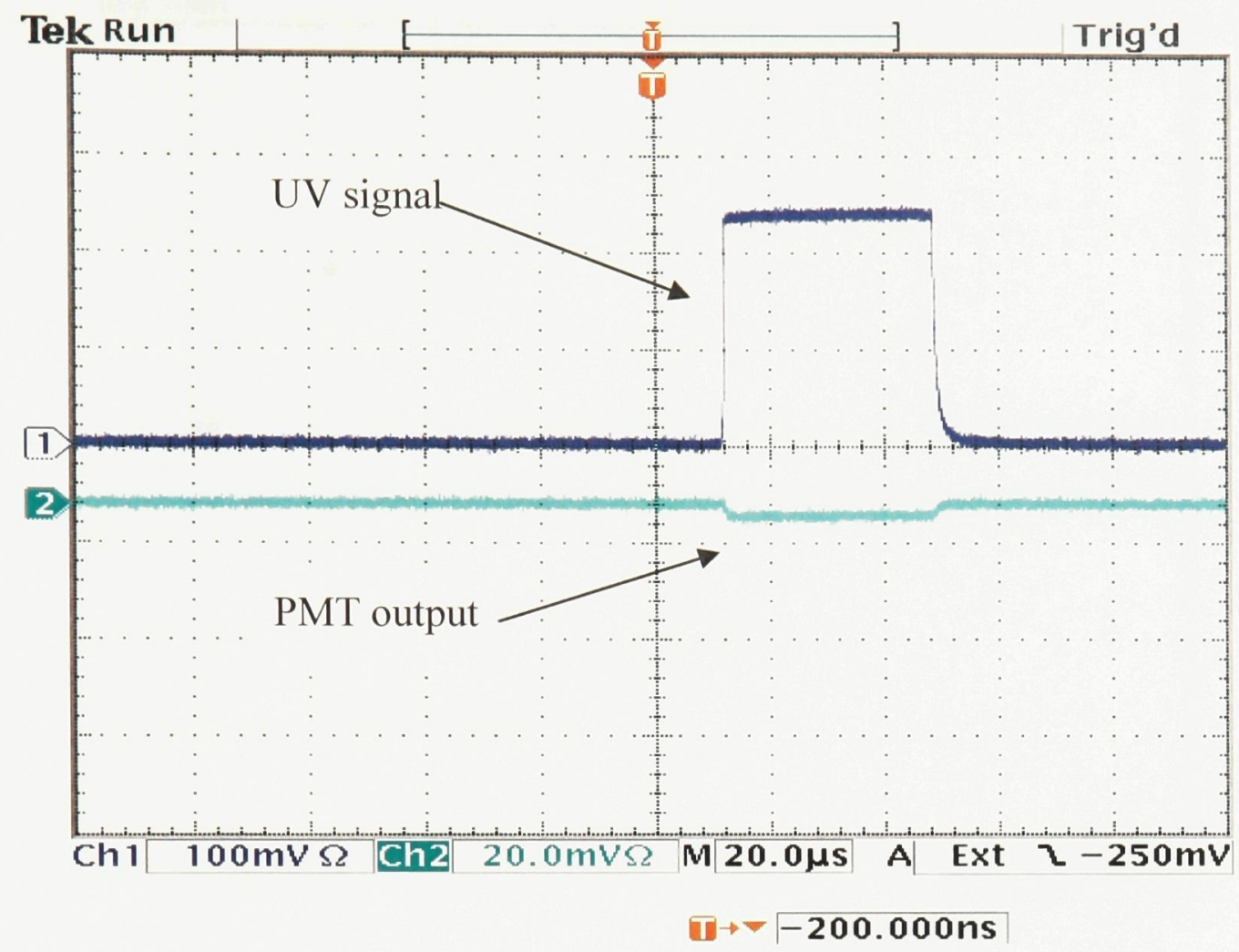

Figure 4.2.3 PMT output with 20V gating

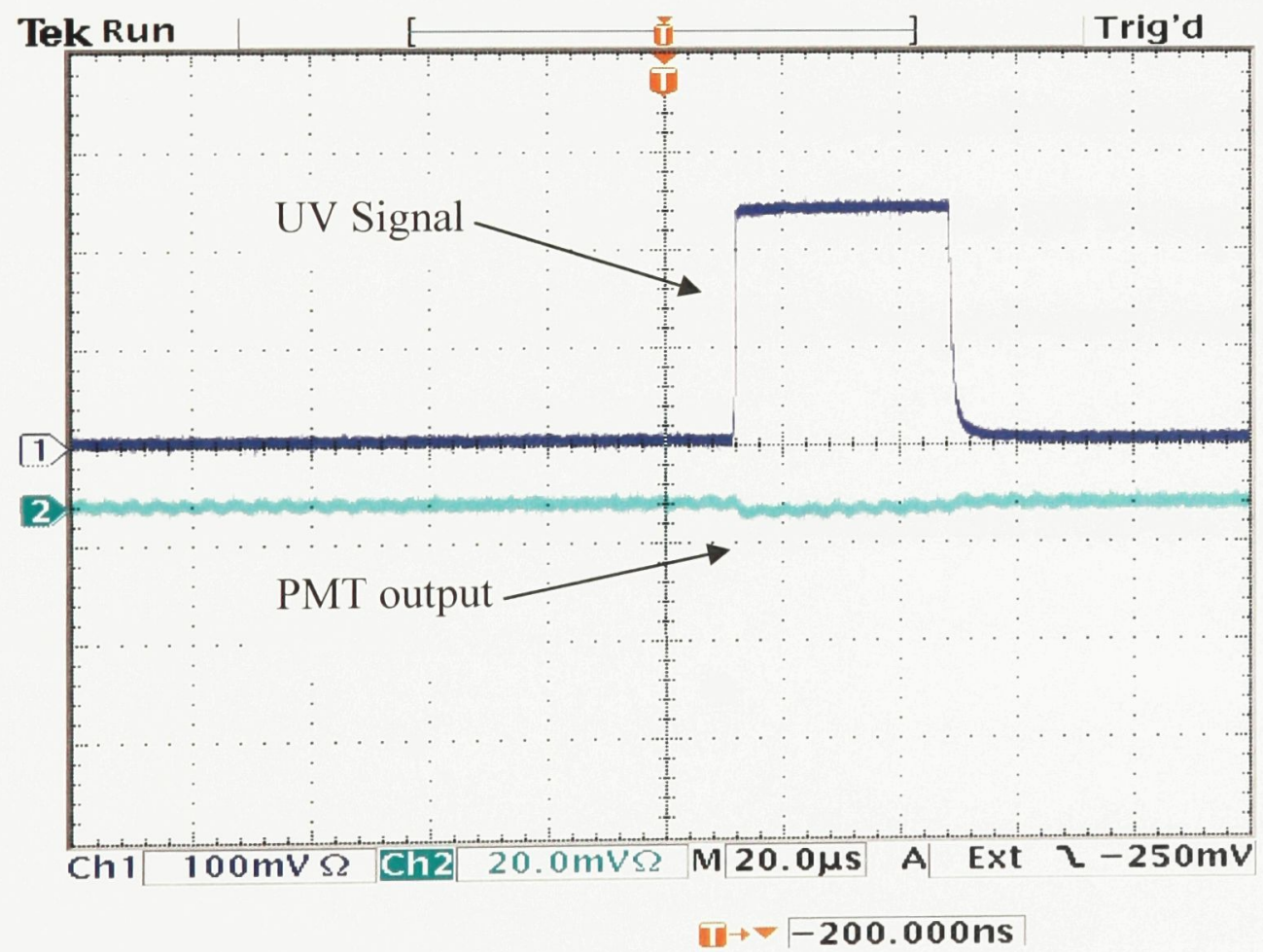

Figure 4.2.4 PMT output with $80 \mathrm{~V}$ gating 


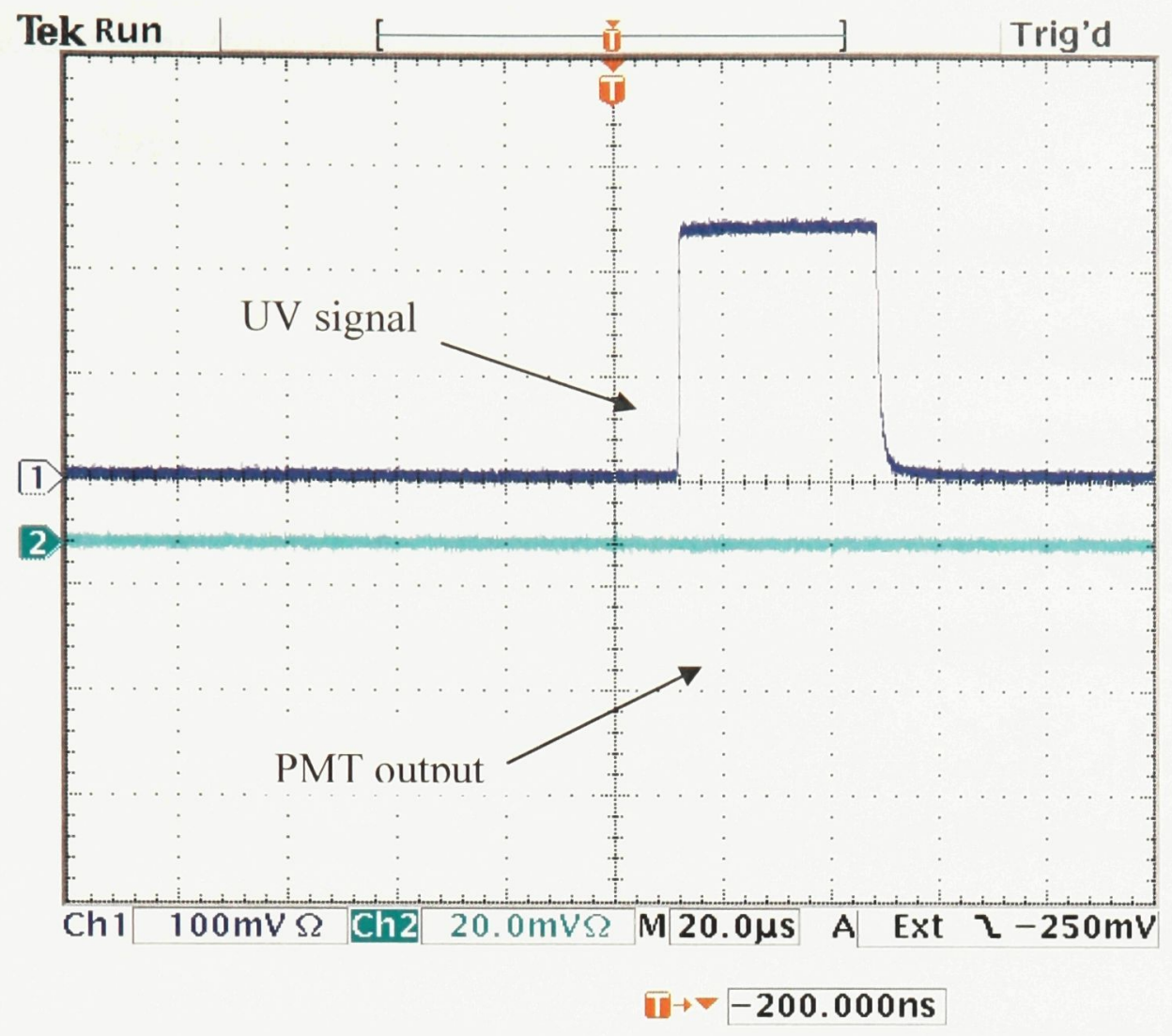

Figure 4.2.5 PMT out put with $100 \mathrm{~V}$ gating voltage

Then the gating signal was connected and a gating voltage of $20 \mathrm{~V}$ was applied. As shown in figure 4.2.3 the output signal of the PMT dropped to about $5 \mathrm{mV}$. With increase of the gating voltage to $80 \mathrm{~V}$ the signal shrank more and at $100 \mathrm{~V}$ the amplitude of the signal was almost zero (figures 4.2.4 and 4.2.5). These preliminary results proved that the gating circuit was able to shut off the PMT.

The next step was to evaluate the gating circuit with scattered $\mathrm{x}$ rays from a clinical linear accelerator. 


\subsection{Evaluating the gating circuit with a linear accelerator and obtaining the optimal gating voltage and gating width}

At this stage the same setup we had in the lab was transferred to National Research Council Canada and the gating circuit was tested with scattered $\mathrm{x}$ rays from linear accelerator, Elekta Precise (the UV filter was removed and BGO crystal was directly coupled to the PMT with silicon grease). The goal was to prove that gating circuit is operational and also to obtain an optimal gating width and gating voltage to have an acceptable energy resolution.

Two energy levels of x-ray were tested, $6 \mathrm{MV}$ and $10 \mathrm{MV}$. The output dose rate from linac is called monitor unit (MU) rate. The monitor unit rate is in the unit of MU/min. One monitor unit corresponds to about $1 \mathrm{cGy}$ dose at the isocenter. For the Linac which was used, the intensity of each x-ray pulse is fixed and the monitor unit rate is adjusted through the x-ray pulse frequency. The x-ray pulse frequency can be $25,50,100,200$, and $400 \mathrm{~Hz}$ (6 MV only). For each beam energy the evaluation was done for different monitor unit rates and different frequencies from $25 \mathrm{~Hz}$ to $400 \mathrm{~Hz}$. We also tested different field sizes, from $4 \times 4 \mathrm{~cm}^{2}$ to $15 \times 15 \mathrm{~cm}^{2}$. As shown in figure 4.3 .1 (a), a $30 \times 30$ $\mathrm{cm}^{2}$ and $20 \mathrm{~cm}$ thick solid water phantom was placed at $100 \mathrm{~cm}$ SSD (Source to Surface Distance) and isocenter was at 110 from the X-ray source. The detector unit was first aligned to the isocentre at $50 \mathrm{~cm}$ and 90 degrees. Figure 4.3.1 (b) shows the Electronics schematic. The PMT was connected to the high voltage supply and the voltage was set to 830 volts. A signal from the Linac, which indicates the beginning of each x-ray pulse,

was used to trigger the gating circuit. The ${ }^{22} \mathrm{Na}$ source, a positron emission isotope, was sitting on top of the housing of the crystal and PMT. 


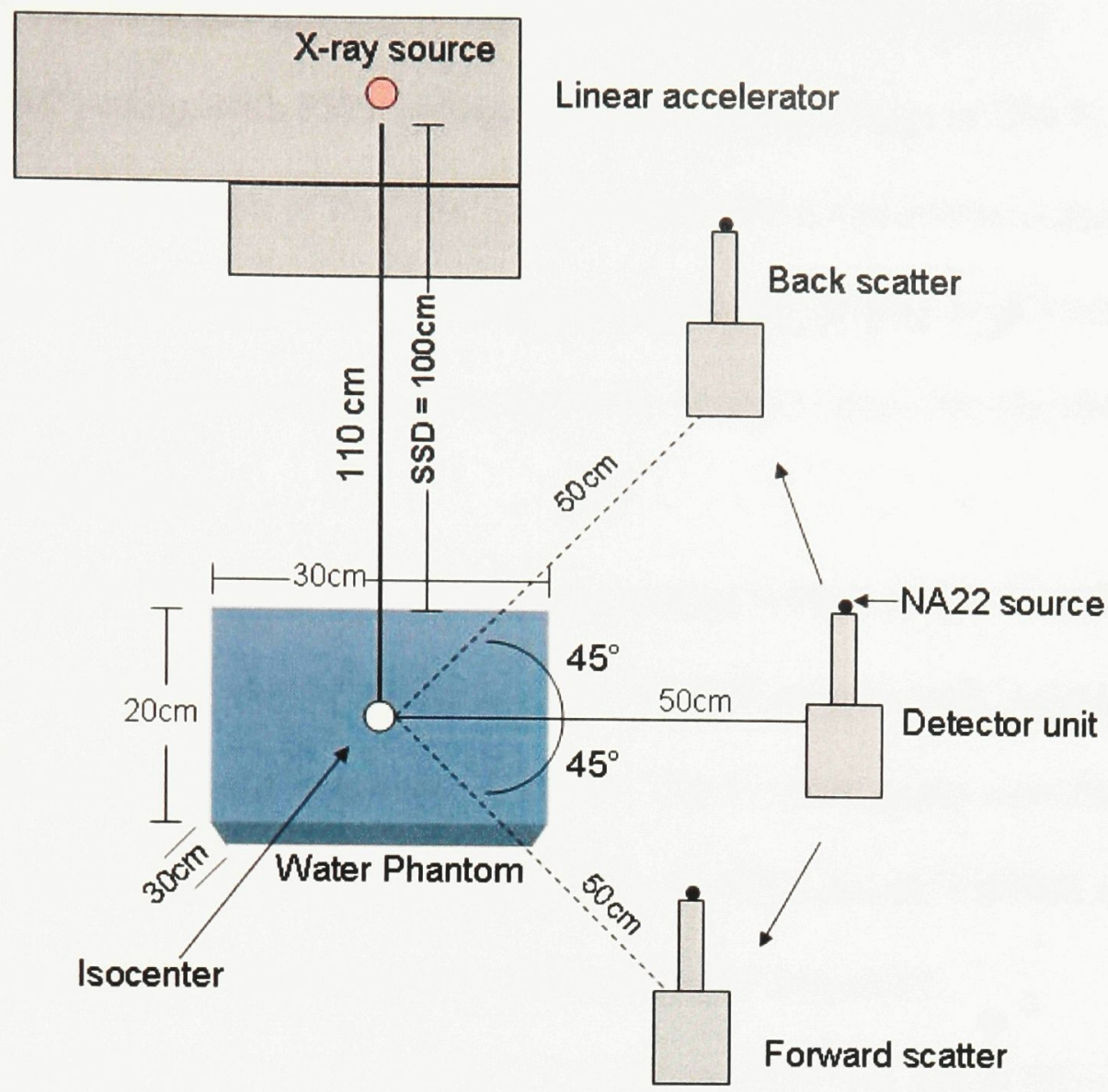

(a)

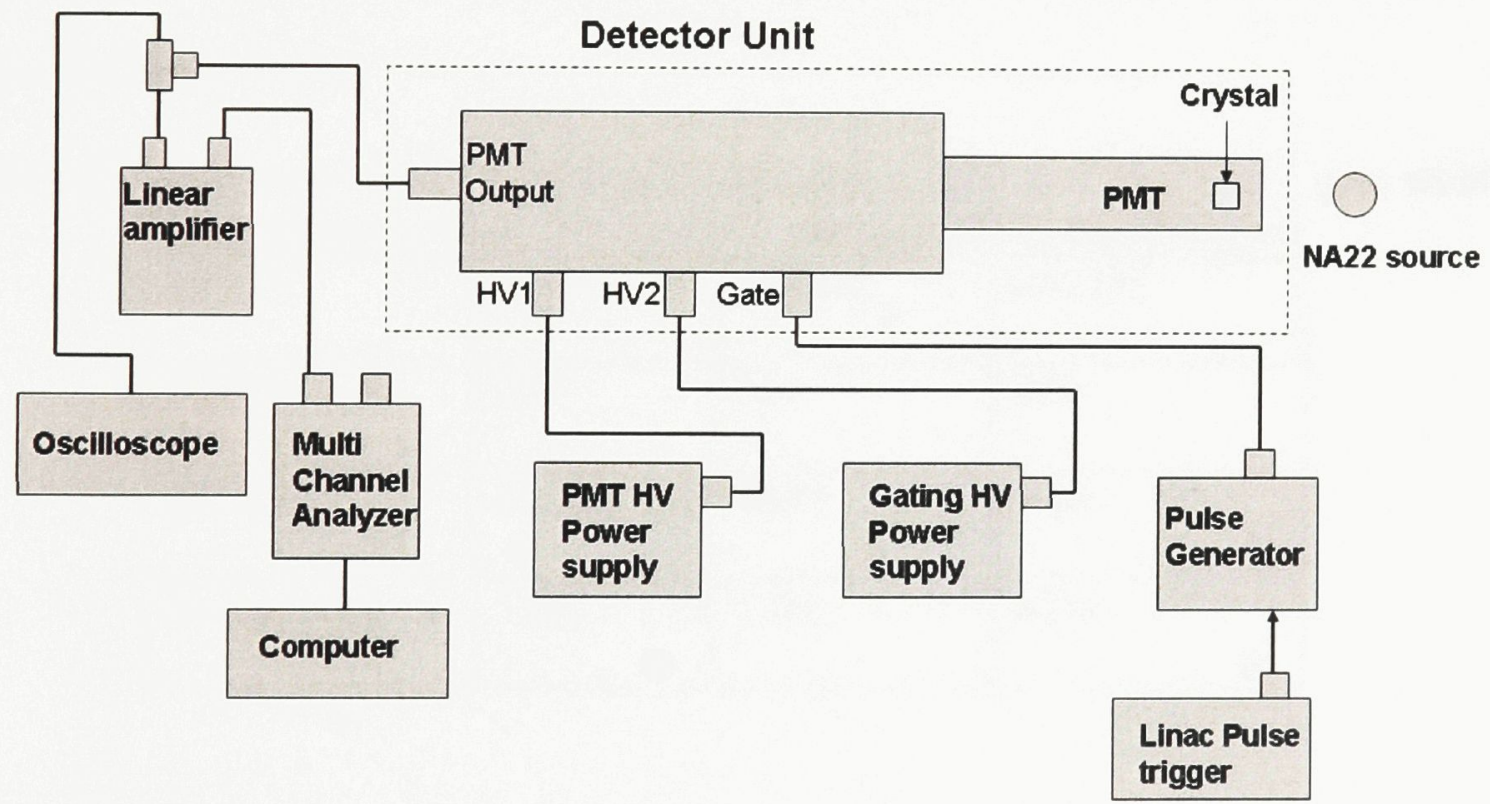

(b)

Figure 4.3.1 Geometry (a) and electronics schematic (b) of the detector unit setup with the Linac at NRC. 
$10 \mathrm{MV}, 15 \times 15 \mathrm{~cm}^{2}$ field size (gating voltage: $200 \mathrm{~V}$, gating width $150 \mu \mathrm{s}$ )

At 10 MV setting, with PMT voltage of $830 \mathrm{~V}$, gating voltage of $200 \mathrm{~V}$, and gate width of $150 \mu \mathrm{s}$, the spectrum of the ${ }^{22} \mathrm{Na}$ was recorded with a multichannel analyzer, MCA, for different frequencies, therefore different monitor unit rates, with $15 \times 15 \mathrm{~cm}^{2}$ field size. Figure 4.3.2 shows the spectrum for $50 \mathrm{~Hz}$ : $95 \mathrm{MU} / \mathrm{min}, 100 \mathrm{~Hz}: 194 \mathrm{MU} / \mathrm{min}$ and $200 \mathrm{~Hz}: 391 \mathrm{MU} / \mathrm{min}$.

It can be seen that for the gate width of $150 \mu$ s and gating voltage of $200 \mathrm{~V}$, at $200 \mathrm{~Hz}$ the spectrum is not as good as lower frequencies. The $511 \mathrm{keV}$ gamma peak is almost lost in the background and is not distinguishable anymore. The $511 \mathrm{keV}$ peaks were fitted with a Gaussian function and the results, the center of the peak (XC) and the FWHM, are shown in table 4.1. The energy resolution is much worse at higher frequency.

It should be noted that $15 \times 15 \mathrm{~cm}^{2}$ field size is a fairly big field size, and at this size of a tumor, the tumor motion is unlikely a concern. This large size field was chosen to simulate the worst scatter.

Table 4.1 Energy resolution for different frequencies: field size $15 \times 15 \mathrm{~cm}^{2}$, gate width $150 \mu$ s, gating voltage $200 \mathrm{~V}$, PMT voltage $830 \mathrm{~V}, 10 \mathrm{MV}$ beam.

\begin{tabular}{|l|l|l|l|}
\hline $10 \mathrm{MV}$ & $50 \mathrm{~Hz}$ & $100 \mathrm{~Hz}$ & $200 \mathrm{~Hz}$ \\
\hline FWHM (ch) & 255 & 349 & 466 \\
\hline XC (ch) & 1668 & 1494 & 1066 \\
\hline Energy Res & $15 \%$ & $23 \%$ & $44 \%$ \\
\hline
\end{tabular}




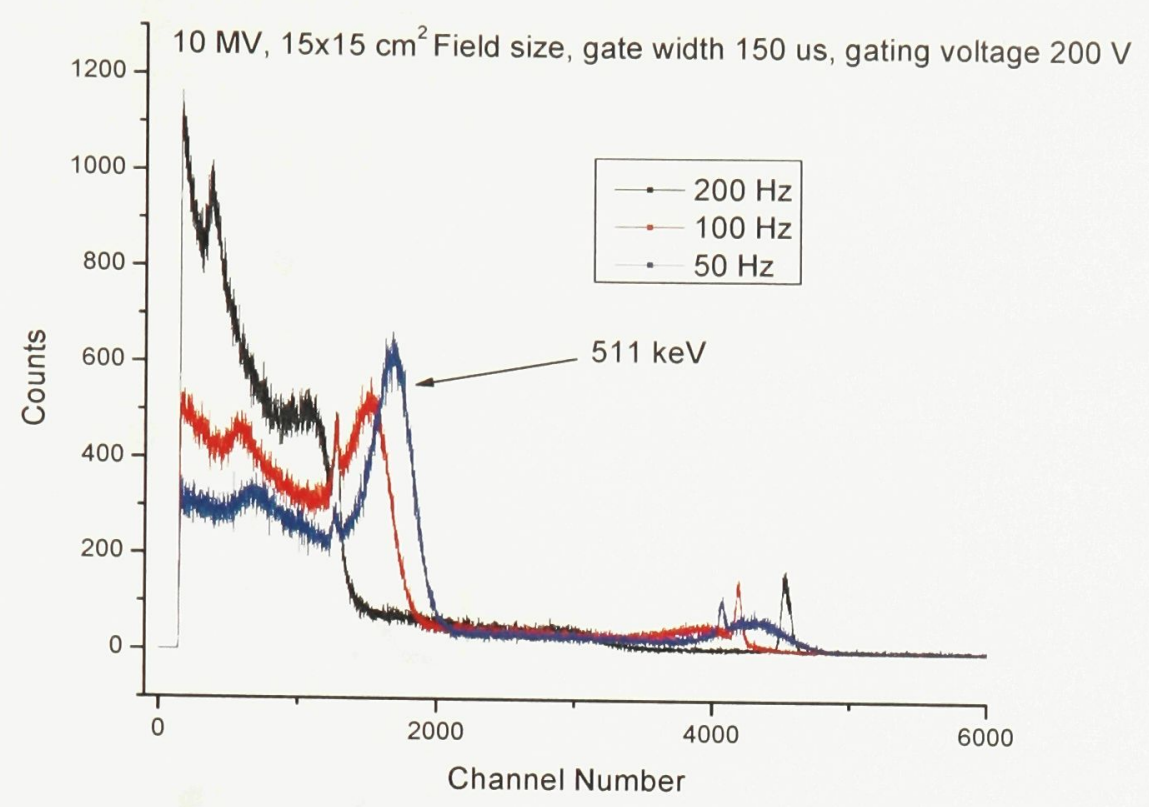

Figure 4.3.2 Energy spectrum of ${ }^{22} \mathrm{Na}$, in the radiation environment of a Linac, with gating.

\section{$6 \mathrm{MV}, 15 \times 15 \mathrm{~cm}^{2}$ field size, (gating voltage: $200 \mathrm{~V}$, gating width $150 \mu \mathrm{s}$ )}

The same experiment was performed with the $6 \mathrm{MV}$ beam as well, and the result is presented in figure 4.3.3. The $511 \mathrm{keV}$ peaks were fitted with a Gaussian function and results are shown in Table 4.2.

Table 4.2 Energy resolution for different frequencies: field size $15 \times 15 \mathrm{~cm}^{2}$, gate width $150 \mu$ s, gating voltage $200 \mathrm{~V}$, PMT voltage $830 \mathrm{~V}, 6 \mathrm{MV}$ beam.

\begin{tabular}{|l|l|l|l|}
\hline 6MV & $50 \mathrm{~Hz}$ & $100 \mathrm{~Hz}$ & $200 \mathrm{~Hz}$ \\
\hline FWHM (ch) & 318 & 393 & 355 \\
\hline XC (ch) & 1726 & 1576 & 1124 \\
\hline Eng Res & $18 \%$ & $25 \%$ & $32 \%$ \\
\hline
\end{tabular}




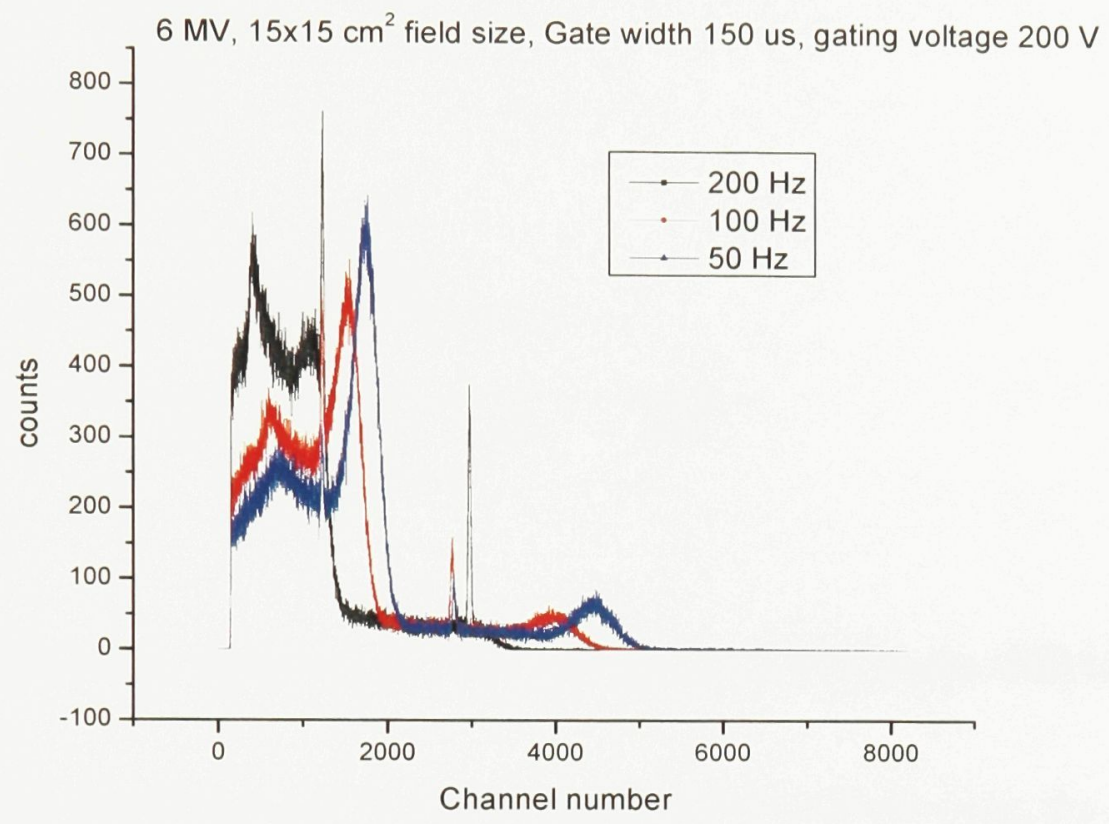

Figure 4.3.3 Energy spectrum of ${ }^{22} \mathrm{Na}$, in the radiation environment of a Linac, with gating.

The same problem of having bad energy resolution at higher frequencies was observed.

There are some additional sharp peaks shown up on the spectrum (around channel number 1000 and 3000 for black spectrum), which are related to the switching noise from the gating circuit. These noises can be rejected by the data acquisition system.

\section{The effect of field size:}

The next step was to change the field size and see the effects on the spectrum and energy resolution. The PMT voltage was set to $830 \mathrm{~V}$, the gating voltage was set to 200 $\mathrm{V}$, gate width $150 \mu \mathrm{s}$, and frequency was set to $50 \mathrm{~Hz}$ for $6 \mathrm{MV}$ beam, $47 \mathrm{MU} / \mathrm{min}$. Figure 4.3.4 shows the spectra for two different field sizes. The results of fitting with a Gaussian function for these two and three other field sizes are listed in Table 4.3. 


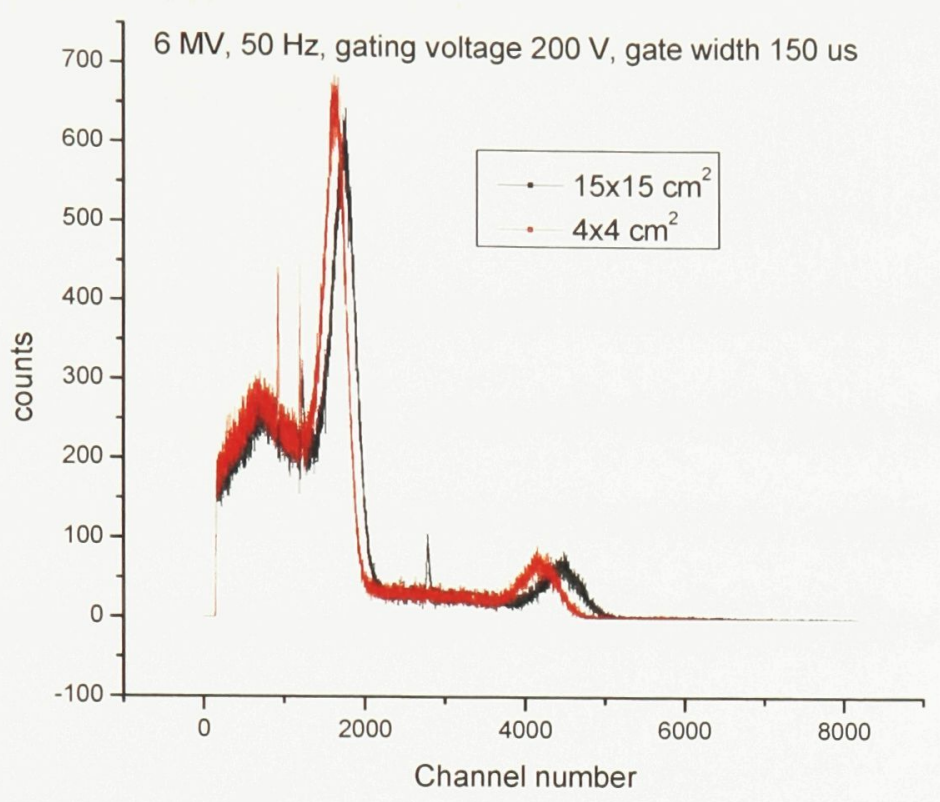

Figure 4.3.4 Energy spectrum of ${ }^{22} \mathrm{Na}$, with gating, $6 \mathrm{MV}$ beam, different field sizes.

It is observed that at this low frequency for all different field sizes, the gating voltage of $200 \mathrm{~V}$ and $150 \mu \mathrm{s}$ width, is sufficient and the spectra have good peaks with energy resolution of $21 \%$.

Table 4.3 Energy resolution for different field sizes.

\begin{tabular}{|l|l|l|l|l|l|}
\hline $50 \mathrm{~Hz}, 6 \mathrm{Mv}$ & $15 \times 15 \mathrm{~cm}^{2}$ & $10 \times 10 \mathrm{~cm}^{2}$ & $8 \times 8 \mathrm{~cm}^{2}$ & $6 \times 6 \mathrm{~cm}^{2}$ & $4 \times 4 \mathrm{~cm}^{2}$ \\
\hline FWHM (ch) & 367 & 354 & 353 & 348 & 345 \\
\hline XC (ch) & 1734 & 1653 & 1635 & 1626 & 1627 \\
\hline Eng Res & $21 \%$ & $21 \%$ & $21 \%$ & $21 \%$ & $21 \%$ \\
\hline
\end{tabular}

As the scatter level is approximately proportional to the field size (in area), these results indicate that the scatter level has little effect on the performance of the detector.

The above results show that the gating frequency, rather than the amount of scattered, has significant effect on the performance of detector. Therefore, the next step 
was to obtain the optimal gating parameters, i.e. gate width and gating voltage.

\section{Optimal gate width}

$-50 \mu \mathrm{s}$

To find the optimal gate width, the width of the gate was reduced from $150 \mu$ s to $50 \mu$ s and spectra were obtained with $6 \mathrm{MV}$ beam, at $200 \mathrm{~Hz}$, gating voltage $200 \mathrm{~V}$ and $10 \times 10 \mathrm{~cm}^{2}$ field size. Figure 4.3.5 shows the spectra. The result of fitting the $511 \mathrm{keV}$ peaks to a Gaussian function is also listed in Table 4.4.

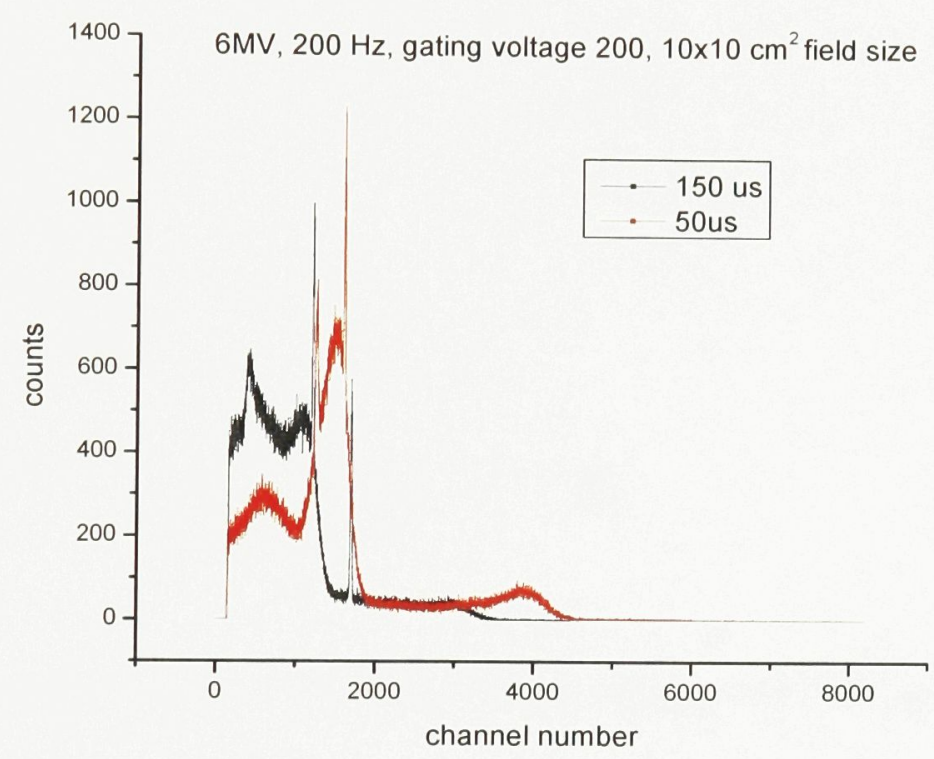

Figure 4.3.5 Energy spectrum of ${ }^{22} \mathrm{Na}$, with gating, $6 \mathrm{MV}$ beam, different gate width.

Table 4.4 Energy resolution for different gate width.

\begin{tabular}{|c|c|c|}
\hline $200 \mathrm{~Hz}, 6 \mathrm{MV}$ & $150 \mu \mathrm{s}$ & $50 \mu \mathrm{s}$ \\
\hline FWHM (ch) & 381 & 391 \\
\hline $\mathrm{XC}$ (ch) & 1097 & 1481 \\
\hline Energy Resolution & $35 \%$ & $26 \%$ \\
\hline
\end{tabular}


It was observed that spectrum and therefore energy resolution is much better with shorter gate width.

Encouraged by these results, we increased the frequency to $400 \mathrm{~Hz}$ and repeated the experiment. At $400 \mathrm{~Hz}$, with the long gate $(150 \mu \mathrm{s})$ the spectrum was completely distorted for different field sizes. Even for zero field size the $511 \mathrm{keV}$ peak was not distinguishable from the background. Figure 4.3.6 shows the spectrum for zero field size at $400 \mathrm{~Hz}$ with long gate width of $150 \mu \mathrm{s}$.

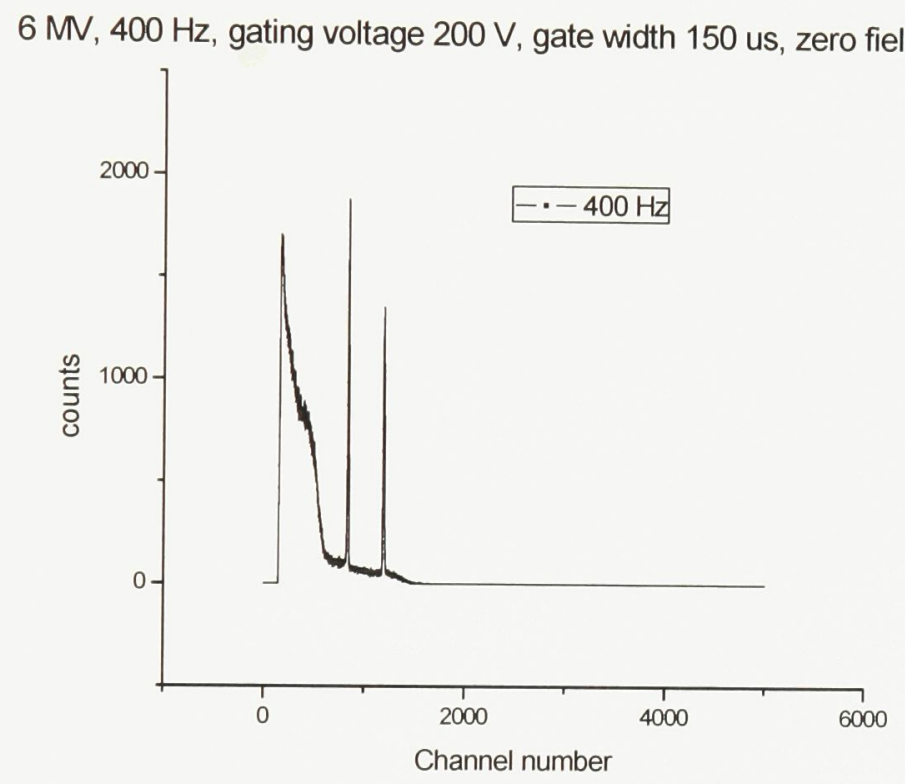

Figure 4.3.6 Energy spectrum of ${ }^{22} \mathrm{Na}, 150 \mu$ s gating, $6 \mathrm{MV}$ beam, $400 \mathrm{~Hz} \mathrm{x}$-ray signal.

After changing the gate width to $50 \mu$ s, we were able to obtain spectra for different field sizes and they all had acceptable energy resolution and good $511 \mathrm{keV}$ peaks. Figure 4.3 .7 shows one of these spectra at $400 \mathrm{~Hz}$ for a $10 \times 10 \mathrm{~cm}^{2}$ field size. Since the shorter gate had encouraging results we reduced the gate width even lower to 30 and $3 \mu \mathrm{s}$ and recorded spectra at $400 \mathrm{~Hz}$ for $6 \mathrm{MV}$ beam for $8 \times 8 \mathrm{~cm}^{2}$ field size. Figure 4.3.8 shows the spectra obtained for 50,30 and $3 \mu$ s. The energy resolution was also calculated after fitting the peaks with a Gaussian function and is listed in Table 4.5. 


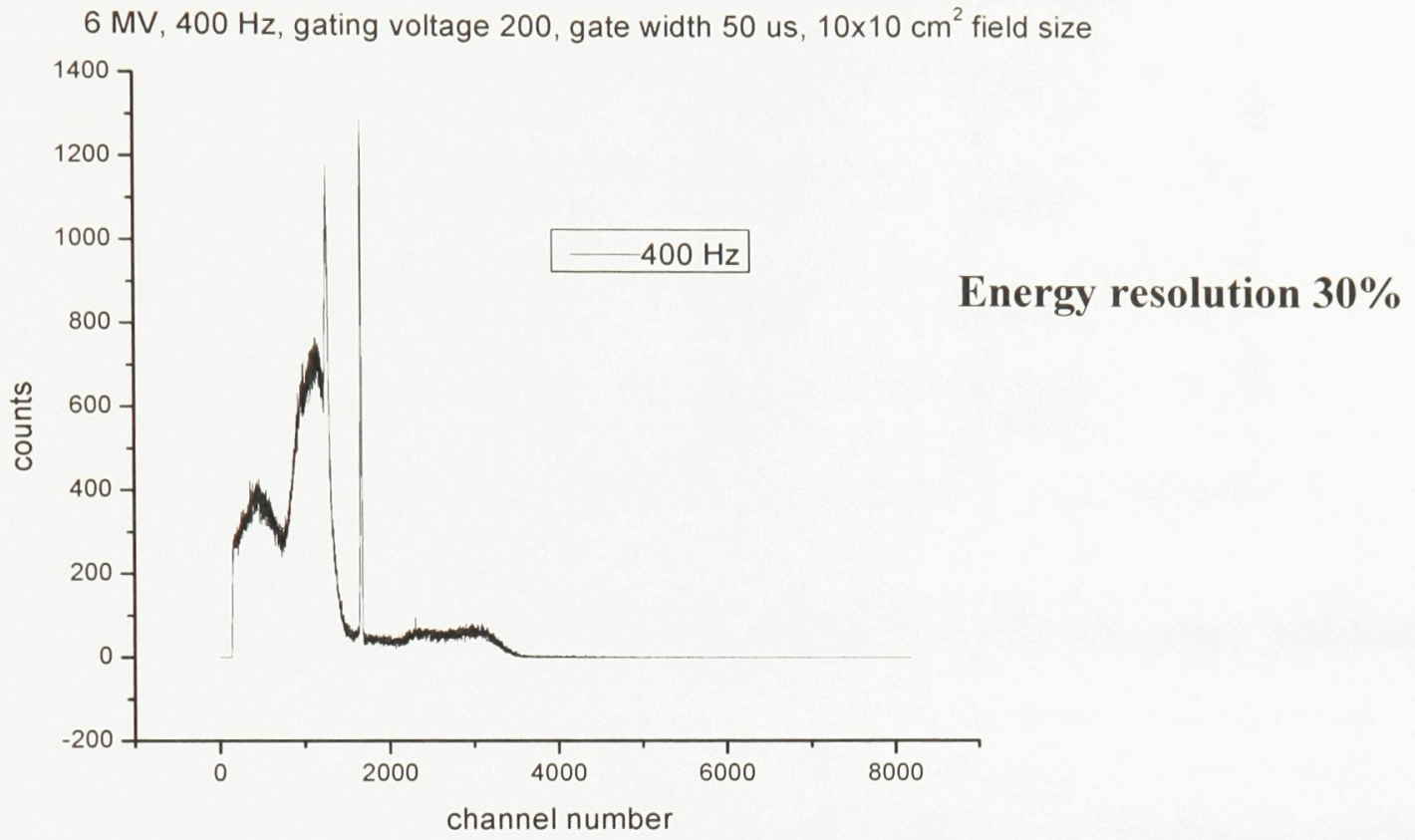

Figure 4.3.7 Energy spectrum of ${ }^{22} \mathrm{Na}, 50 \mu$ s gating, $6 \mathrm{MV}$ beam, $400 \mathrm{~Hz}$ x-ray signal.

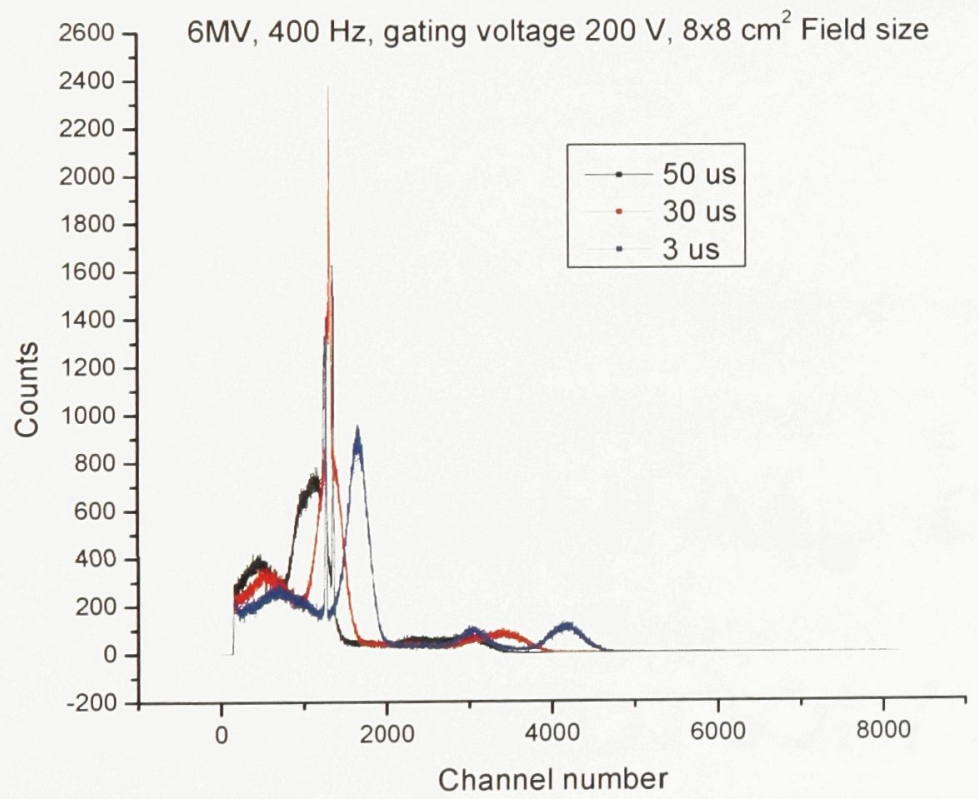

Figure 4.3.8 Energy spectra of ${ }^{22} \mathrm{Na}, 6 \mathrm{MV}$ beam, $400 \mathrm{~Hz}$ x-ray signal, different gate widths. 
Table 4.5 Energy resolutions for different gate width.

\begin{tabular}{|l|l|l|l|}
\hline $6 \mathrm{MV}, 400 \mathrm{~Hz}$ & $3 \mu \mathrm{s}$ & $30 \mu \mathrm{s}$ & $50 \mu \mathrm{s}$ \\
\hline FWHM (ch) & 261 & 350 & 349 \\
\hline XC (ch) & 1637 & 1303 & 1139 \\
\hline Energy Res & $15 \%$ & $26 \%$ & $30 \%$ \\
\hline
\end{tabular}

The definite conclusion was that with reducing the gating width, the energy resolution gets better.

In order to assign the optimal gating width, the width of the Linac signal was considered. The x-ray pulse width of the Linac at NRC was around $2.5 \mu$ s. However, to have the gating system to be able to work with other Linacs, the gate width was chosen to be set at $10 \mu$ s long.

\section{-Optimal gating voltage}

The next step was to find the optimal gating voltage. To do so, in the first set of experiments the original setting, with gate width of $150 \mu \mathrm{s}$, was arranged and the effects of gating voltage on the energy resolution and spectrum were recorded. Later, in the second set of experiments, the output signal of PMT was measured for different gating voltage and results were recorded. 


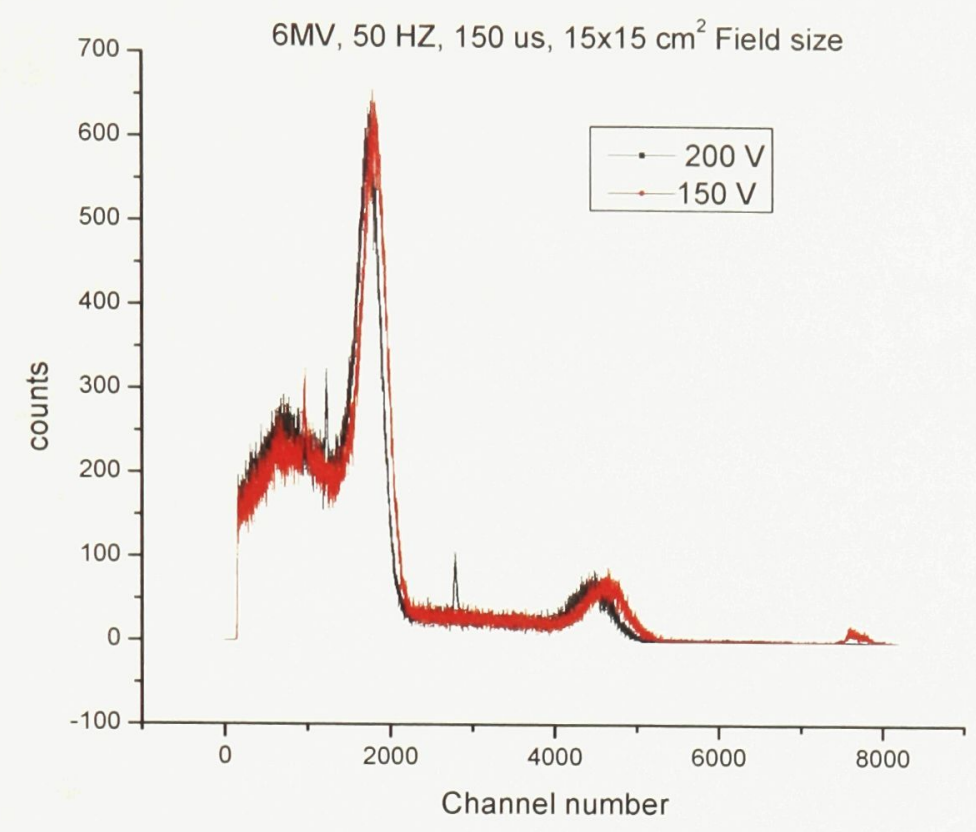

Figure 4.3.9 Energy spectra of ${ }^{22} \mathrm{Na}$ for different gating voltages, $50 \mathrm{~Hz}$.

\section{$-6 \mathrm{MV}, 50 \mathrm{~Hz}, 15 \times 15 \mathrm{~cm}^{2}$, gate width $150 \mu \mathrm{s}$}

At this stage gating voltage was reduced and the effects on the spectrum and energy resolution were measured. The gating voltage was decreased from $200 \mathrm{~V}$ to $150 \mathrm{~V}$ with the same width of 150 so. At $50 \mathrm{~Hz}, 6 \mathrm{MV}$ beam and field size of $15 \times 15 \mathrm{~cm}^{2}$ spectra are shown in figure 4.3.9.

Table 4.6 Energy resolution different gating voltage.

\begin{tabular}{|c|c|c|}
\hline $50 \mathrm{~Hz}, 6 \mathrm{MV}$ & $150 \mathrm{~V}$ & $200 \mathrm{~V}$ \\
\hline FWHM (ch) & 338 & 318 \\
\hline $\mathrm{XC}(\mathrm{ch})$ & 1798 & 1726 \\
\hline Eng Res & $18 \%$ & $18 \%$ \\
\hline
\end{tabular}

The $511 \mathrm{keV}$ peaks were fitted with a Gaussian function. Table 4.6 shows that energy 
resolution of the $511 \mathrm{keV}$ peaks were the same for different gating voltages. In other word, when the Linac is working at $50 \mathrm{~Hz}$, gating voltage does not have any effect on the energy resolution of the $511 \mathrm{keV}$ gamma peaks.

\section{$-6 \mathrm{MV}, 100 \mathrm{~Hz}, 15 \times 15 \mathrm{~cm}^{2}$, gate width $150 \mu \mathrm{s}$}

We also repeated the same experiment for $100 \mathrm{~Hz}$ and $6 \mathrm{MV}$. Figure 4.3 .10 and table 4.7 shows the result of this experiment.

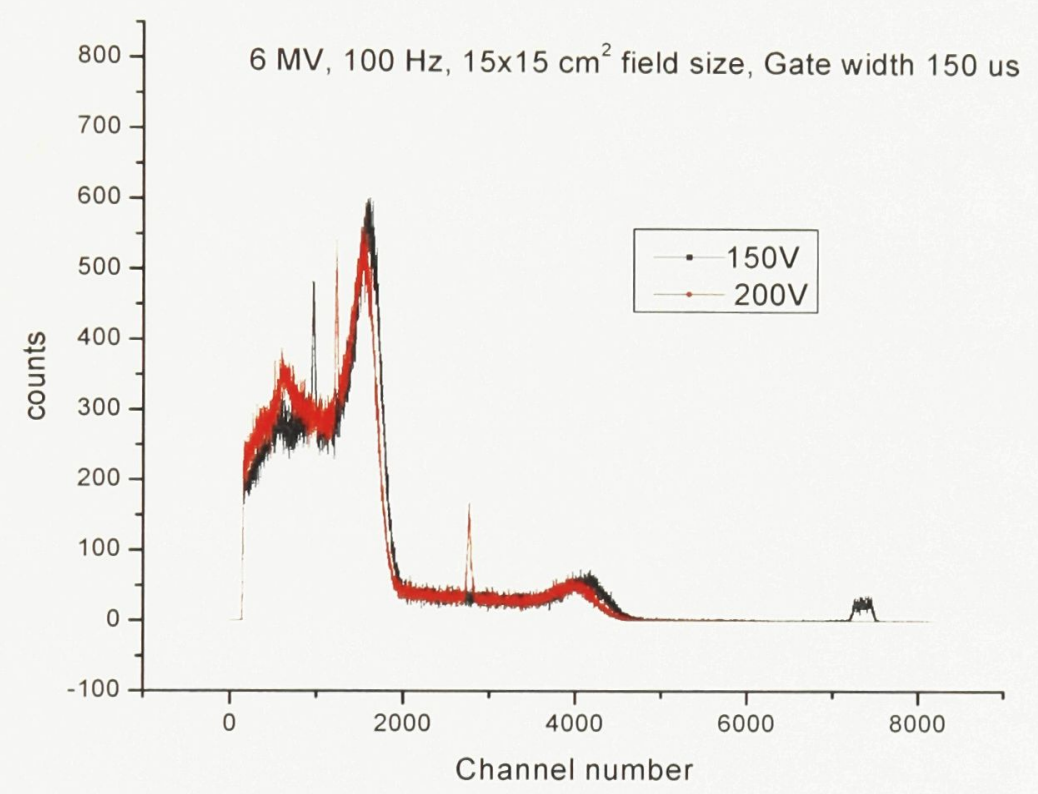

Figure 4.3.10 Energy spectra of ${ }^{22} \mathrm{Na}$ for different gating voltages, at $100 \mathrm{~Hz}$.

Table 4.7 Energy resolution different gating voltage.

\begin{tabular}{|c|c|c|}
\hline $100 \mathrm{~Hz}, 6 \mathrm{MV}$ & $150 \mathrm{~V}$ & $200 \mathrm{~V}$ \\
\hline FWHM (ch) & 393 & 415 \\
\hline XC (ch) & 1576 & 1517 \\
\hline Eng Res & $25 \%$ & $27 \%$ \\
\hline
\end{tabular}

At this frequency, $100 \mathrm{~Hz}$, the lower gating voltage $(150 \mathrm{~V})$ had actually a better energy resolution (25\%) As we increased the frequency, we had better energy resolution for the 
lower gating voltage, i.e. $150 \mathrm{~V}$ compared to $200 \mathrm{~V}$.

It was concluded that at higher frequencies, lower gating voltage will result in a better energy resolution. This result was expected, since lower gating voltage at higher frequencies, will cause less switching noise and is less disturbing to the system electronics.

So far we knew that lowering gating voltage is beneficial and from the experiments on gating width, we had found the optimal gating width of $10 \mu \mathrm{s}$. At this point to find the optimal gating voltage, a second set of experiments was performed with $10 \mu$ s gating width.

Although we knew lower gating voltage is beneficial but it is also known that a lower gating voltage will result in a lower cutoff ratio for PMT output signal. In order to obtain the optimal gating voltage, with the gating width of $10 \mu \mathrm{s}$, the PMT output for different gating voltages were recorded with a Tektronix TDS3012B oscilloscope. While the PMT was connected to a high voltage of $830 \mathrm{~V}$, and Linac working at $50 \mathrm{~Hz}, 6 \mathrm{MV}$ beam, field size of $15 \times 15 \mathrm{~cm}^{2}$, the gating voltage was increased from 0 to $200 \mathrm{~V}$. Figure 4.3.11 shows the gating signal with the gate width $10 \mu$ s and figure 4.3.12 shows the PMT outputs for different gating voltages. As shown before in figure 3.5.3, without gating, PMT is saturated and the PMT output signal of $250 \mathrm{mV}$ shown for zero volts in figure 4.3 .12 can be even higher.

Figure 4.3.12 shows that as we increase the gating voltage, the PMT output signal rapidly decreases. With a gating voltage of 100 volt, the output signal is already reduced from over $250 \mathrm{mV}$ (saturated PMT) to about $50 \mathrm{mV}\left(15 \times 15 \mathrm{~cm}^{2}\right.$ field size), which is low enough for our purpose, i.e. protection of the PMT and the front end electronics. 


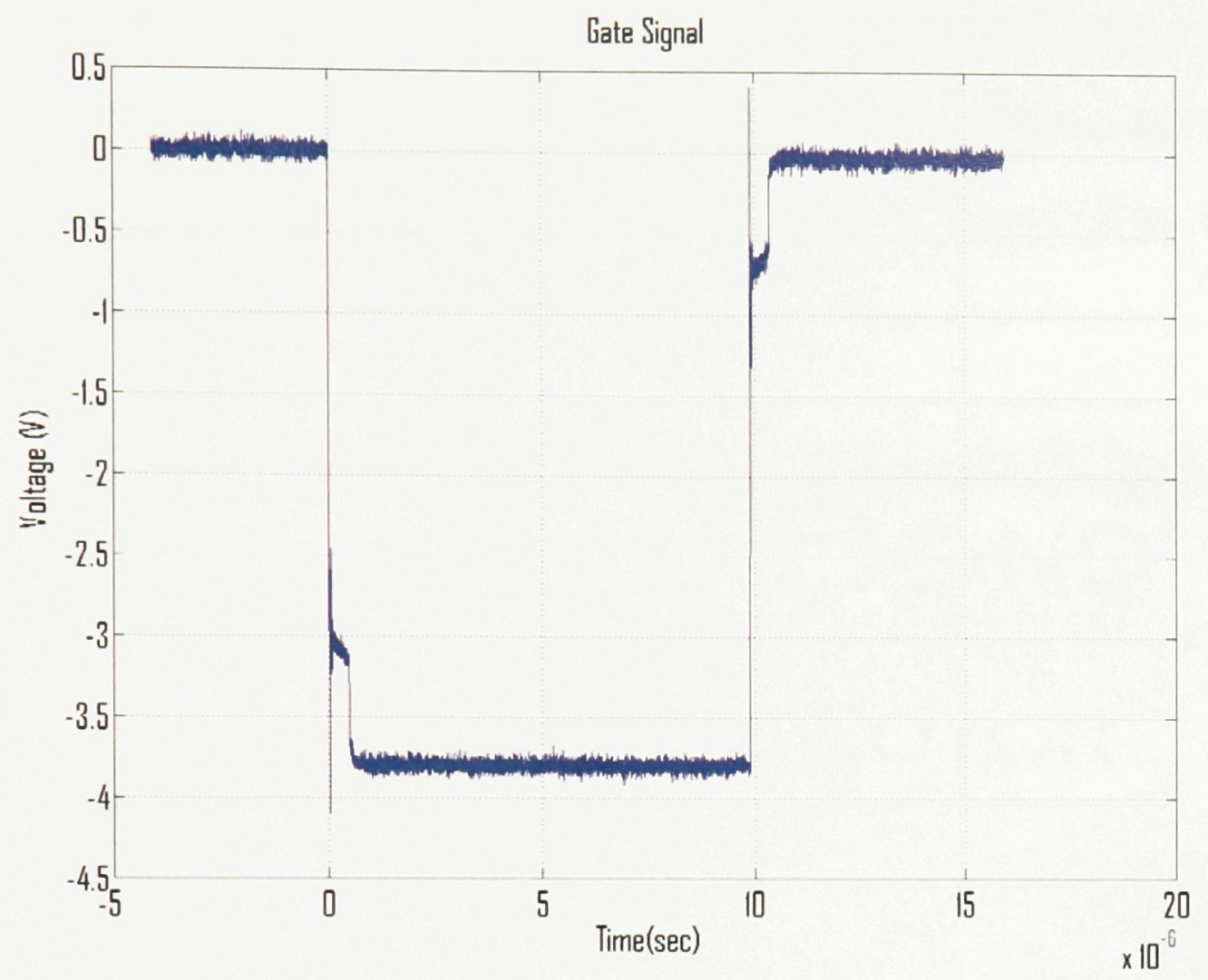

Figure 4.3.11 Gate signal, $10 \mu$ s long.

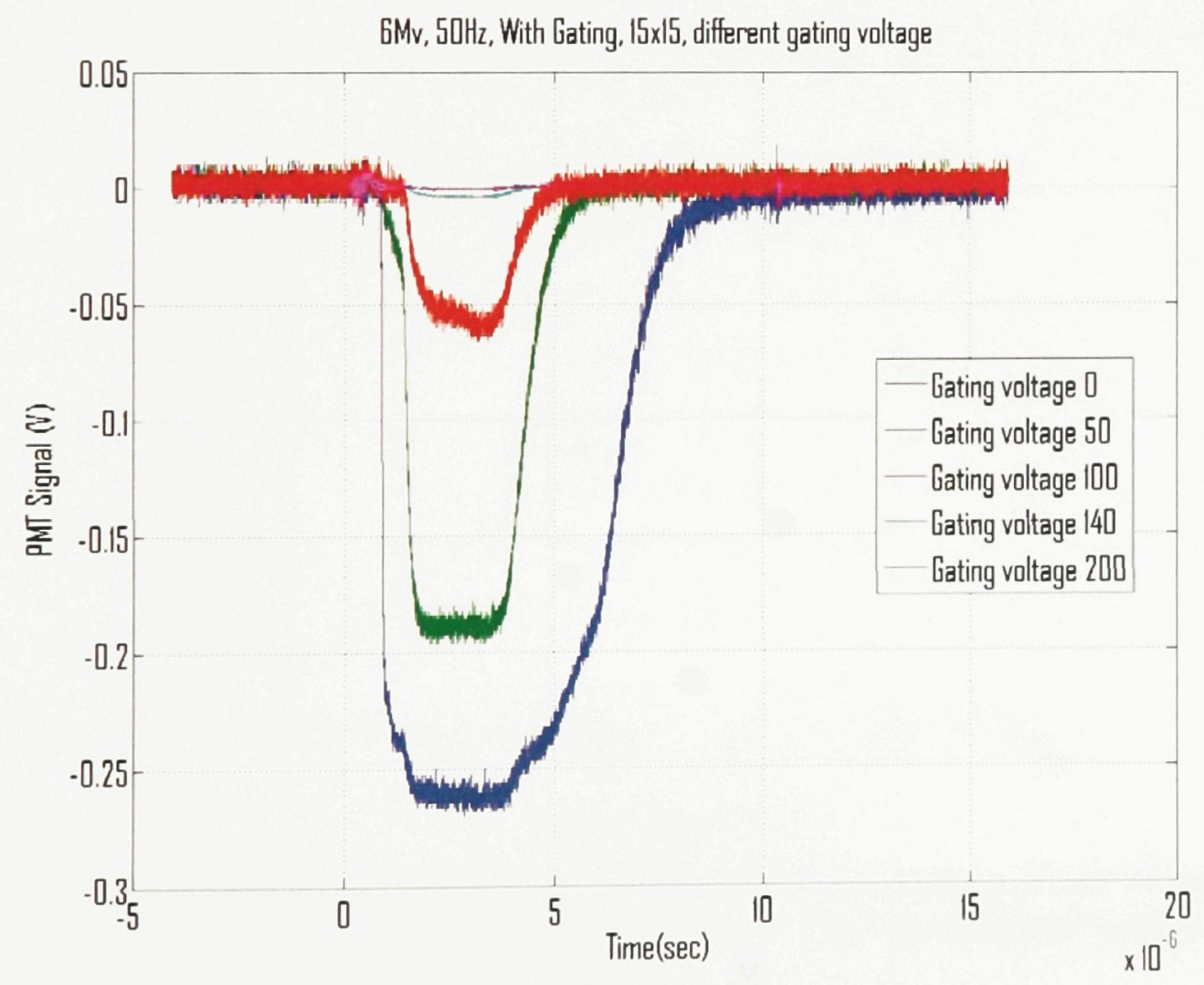

Figure 4.3.12 PMT output signal for different gating voltages. 
With this gating voltage of $100 \mathrm{~V}$ and gating width $10 \mu \mathrm{s}$, the PMT output was recorded for different field sizes at different beam energies. Figure 4.3.13 shows the PMT output with gating applied for 6MV beam at $50 \mathrm{~Hz}$ for different field sizes and figure 4.3.14 shows the same experiment for $10 \mathrm{MV}$ beam.

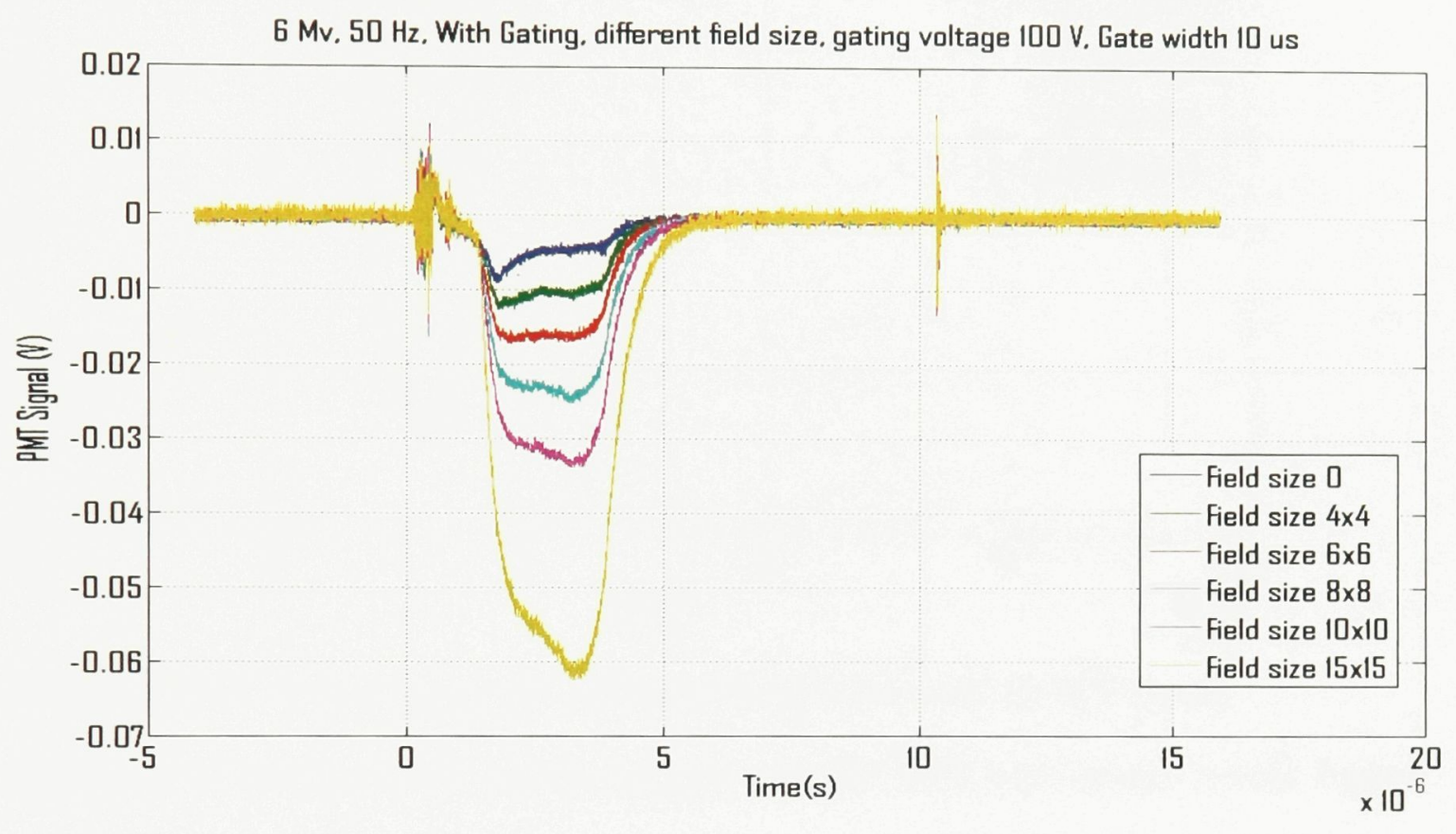

(a)

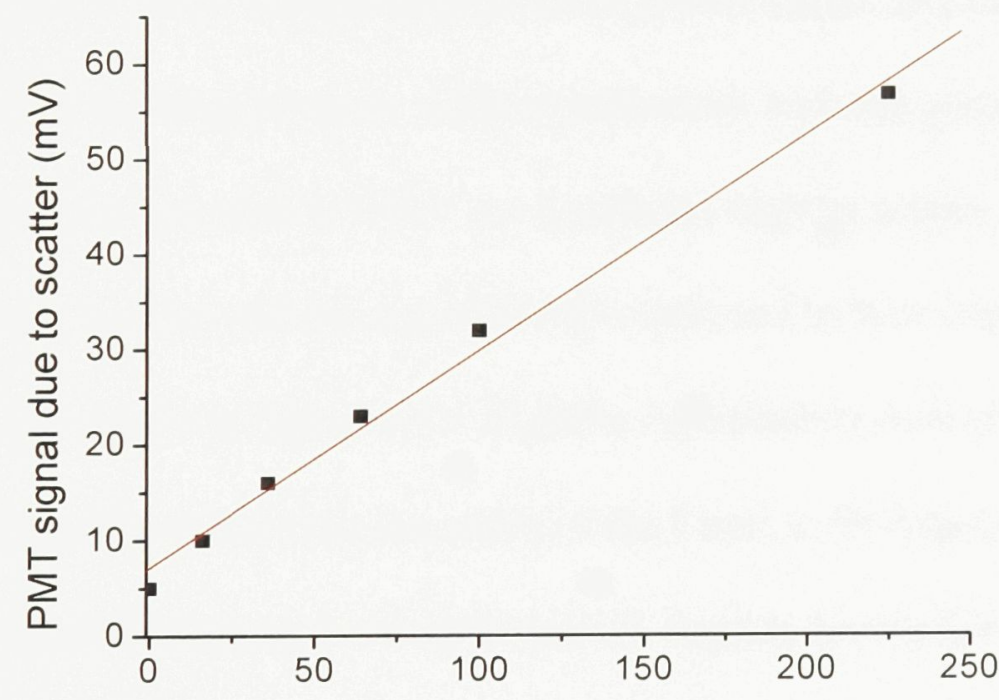

(b)

Field Size $\left(\mathrm{cm}^{2}\right)$

Figure 4.3.13 (a) PMT output signal for different field sizes at $6 \mathrm{MV}$ beam. (b)The amplitude of the PMT signal was plotted against the field size. The line indicates a linear regressing fit. The gating parameters used are $100 \mathrm{~V}$ and $10 \mu \mathrm{s}$. 


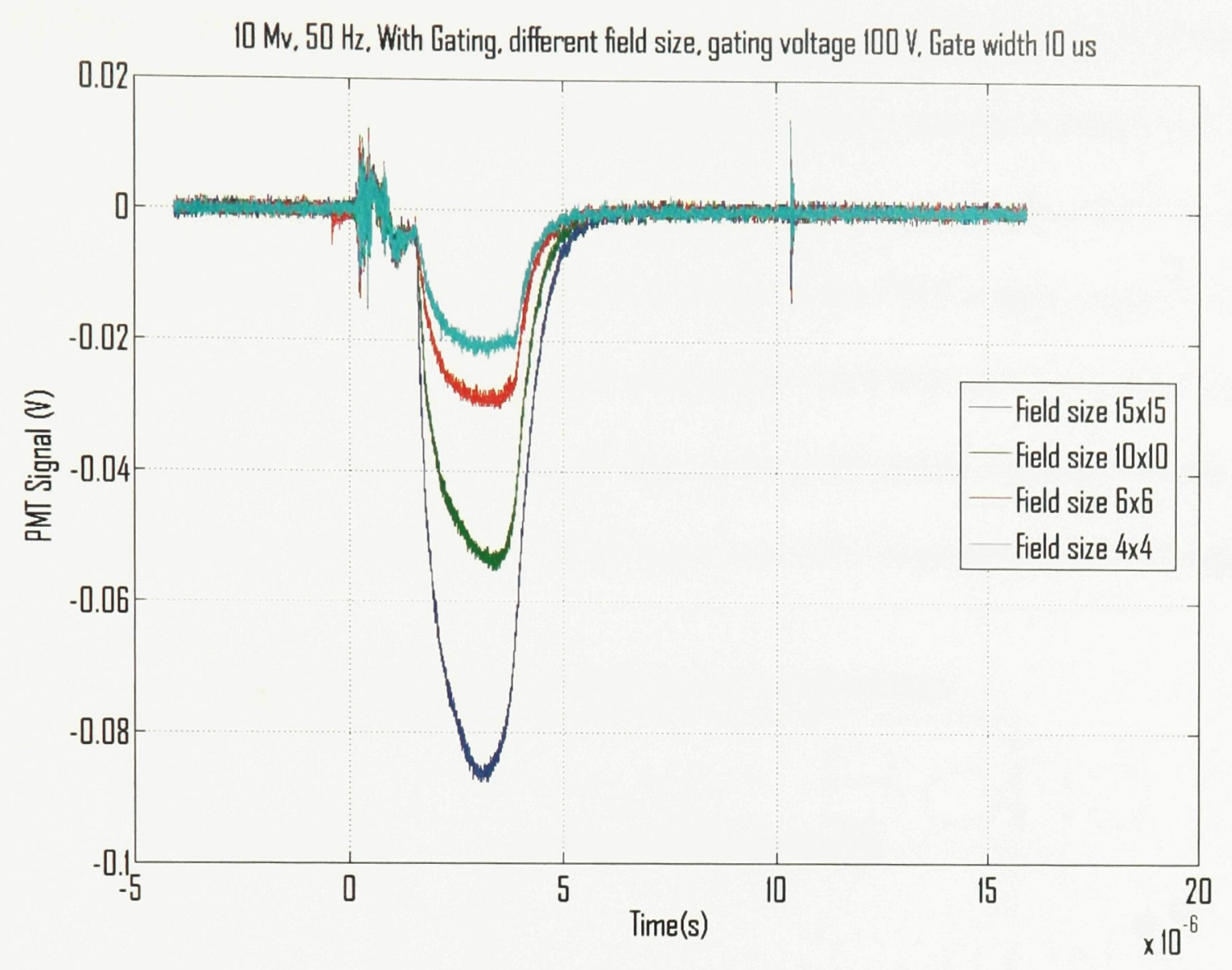

Figure 4.3.14 PMT output signal for different field sizes with $10 \mathrm{MV}$ beam.

These figures show that the PMT signal changes with the field size (scatter level). Figure 4.3.13(b) shows a linear relationship between scatter signal and field size. These indicate that the PMT is working properly without saturation with the gating protection.

The signal level is less than $100 \mathrm{mV}$ even with the highest scatter level $\left(15 \times 15 \mathrm{~cm}^{2}\right.$ field size for $10 \mathrm{MV}$ beam), and this signal level is believed to be tolerable by the front-end electronics. As shown in figure 4.3.1 all these experiments were done while our detector unit was sitting $50 \mathrm{~cm}$ from the isocentre of the Linac at 90 degrees, but scatter level was also measured at 45 degrees backwards and 45 degrees forwards of the phantom for different beam energies.

\section{Backward and Forward Scatter}

The PMT location was also changed to $50 \mathrm{~cm}$ from the isocentre at 45 degrees forward 
(in front of the phantom) and the forward scatter was measured. Figure 4.3.15 shows the PMT output signal for both 6 and $10 \mathrm{MV}$ beams. Location was then change to 45 degrees to the back (behind the phantom, closer to the Linac head) and backscatter was also recorded for both 6 and 10 MV. Figure 4.3.16 shows the PMT output signal for both 6 and $10 \mathrm{MV}$ beam energies As expected, compared with $6 \mathrm{MV}$, at $10 \mathrm{MV}$ there is more scattered radiation at both locations, 45 degrees forward and 45 degrees backward. For both settings of $6 \mathrm{MV}$ and $10 \mathrm{MV}$, there was about 50\% increase in the PMT signal

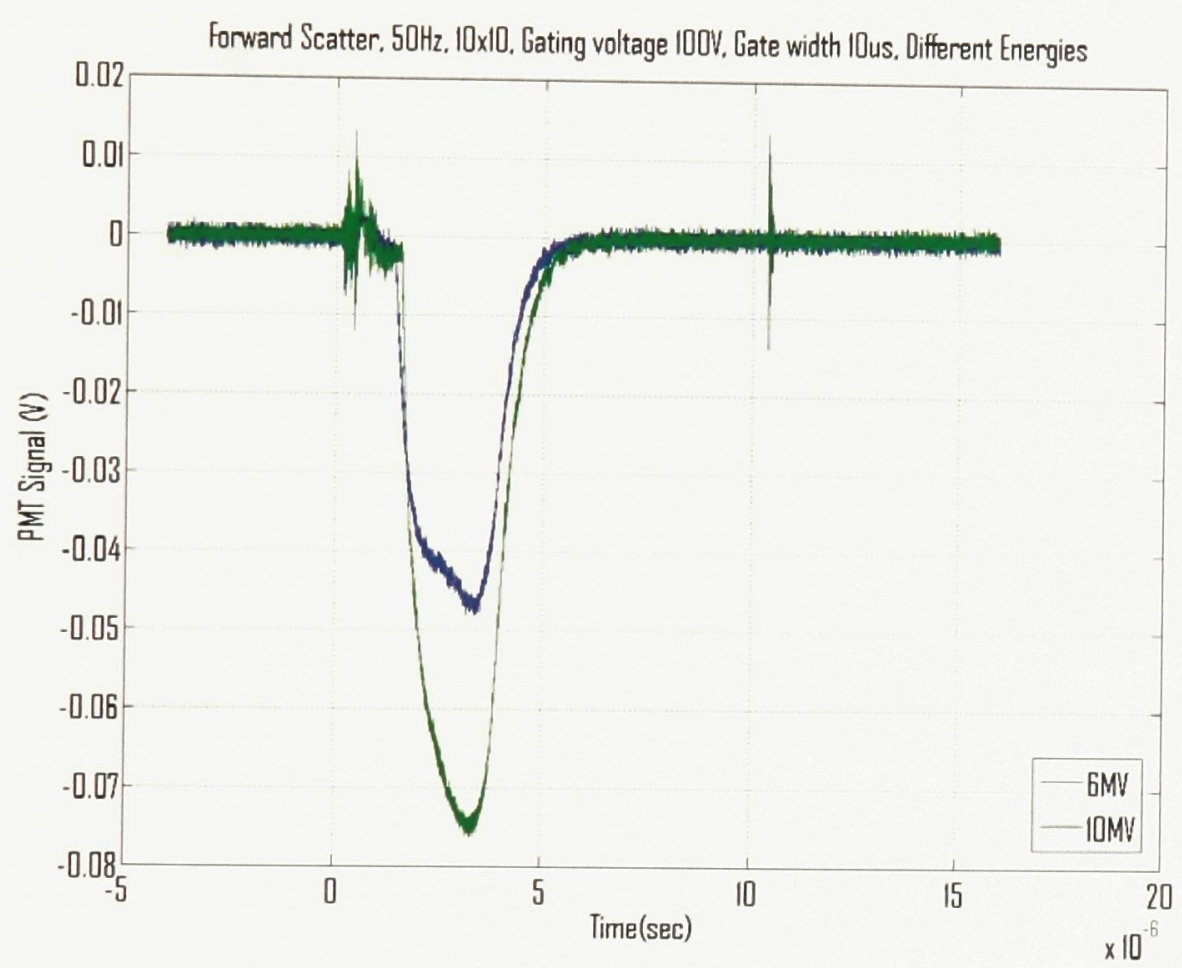

Figure 4.3.15 PMT output for 6 and $10 \mathrm{MV}$ beams at $45^{\circ}$, forward scatter.

between forward scatter position as compared with the backward scatter position.

After all these measurements, optimal gating parameters were fixed at gating width of 10 $\mu$ s and gating voltage of 100 volts. The next step was to measured energy resolutions for different beam energies and Linac frequencies, with a reasonable field size of $8 \times 8 \mathrm{~cm}^{2}$. 


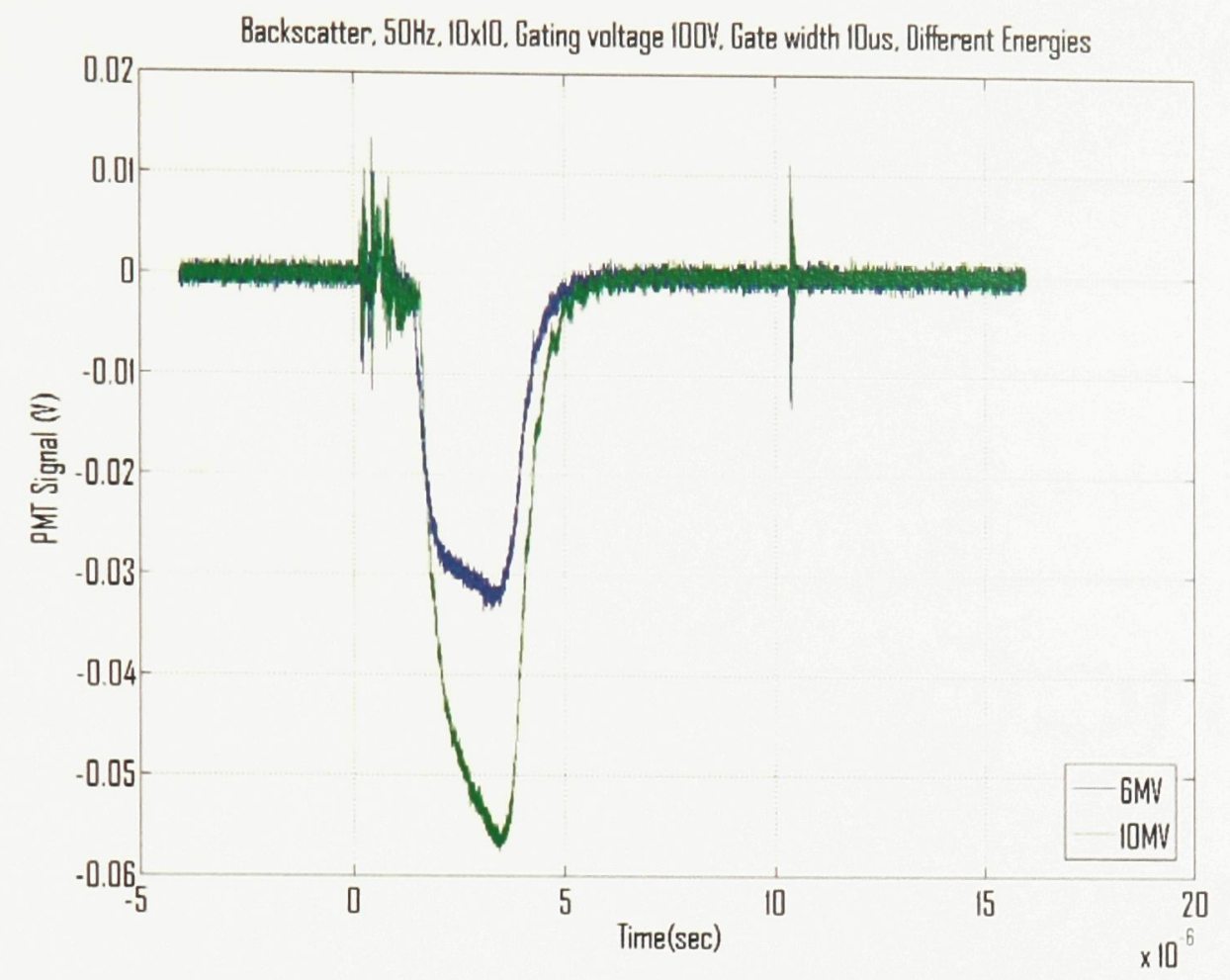

Figure 4.3.16 PMT output for 6 and $10 \mathrm{MV}$ beams at $45^{\circ}$, backscatter.

\subsection{Evaluation of gating with optimized parameters}

The evaluation with the linac was done for different beam energies, $10 \mathrm{MV}$ and $6 \mathrm{MV}$, different frequencies, $25 \mathrm{~Hz}$ to $400 \mathrm{~Hz}$. The optimal gating parameters were selected to be $10 \mu$ s gate width and $100 \mathrm{~V}$ gating voltage. Before applying the gating voltage, a few spectra of our positron emission source, ${ }^{22} \mathrm{Na}$ with $6 \mathrm{MV}$ beam, field size of $8 \times 8 \mathrm{~cm}^{2}$ for different frequencies from 25 to $400 \mathrm{~Hz}$ were obtained. Figure 4.4.1 shows one of these spectra at $400 \mathrm{~Hz}$. Without gating, changing the frequency of the $\mathrm{x}$ ray pulse did not change the spectra that much and we had an energy resolution of $16 \%$ for almost all frequencies. 


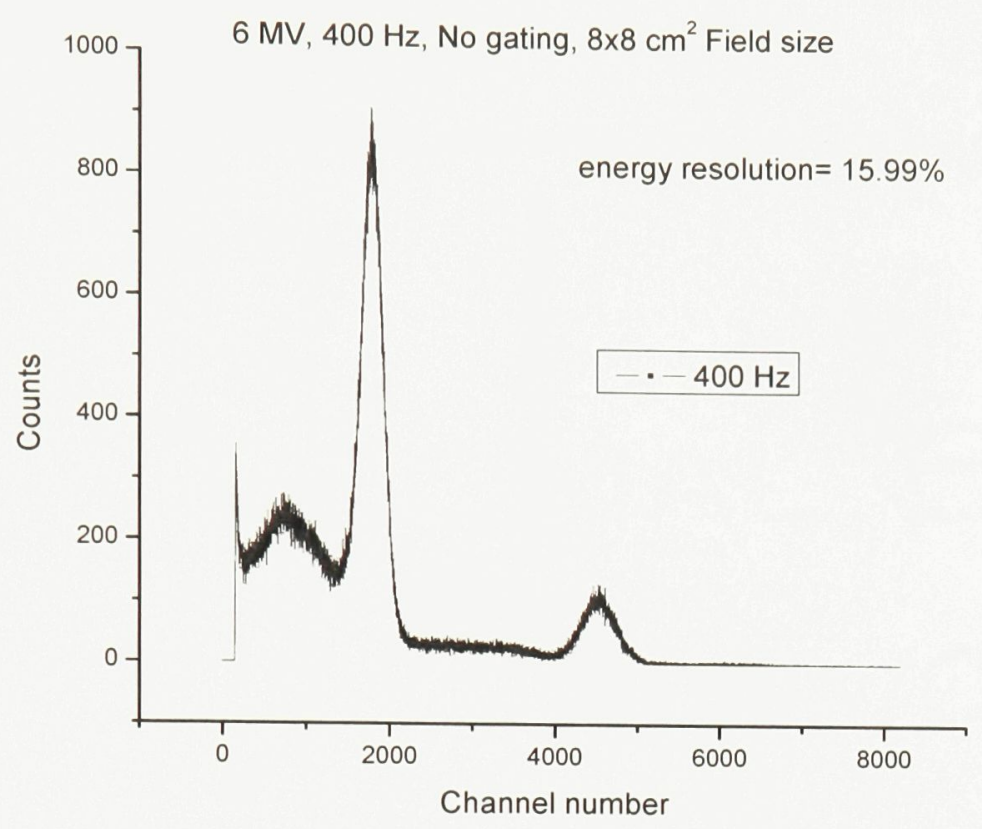

Figure 4.4.1 Energy spectrum of the ${ }^{22} \mathrm{Na}$ without gating at $400 \mathrm{~Hz}$.

After applying the gate, gate width of $10 \mu$ s and voltage of $100 \mathrm{~V}$, spectra were obtained at different frequencies for $6 \mathrm{MV}$ and $10 \mathrm{MV}$ beams.

Figure 4.4.2 shows the spectra for $6 \mathrm{MV}$ beam at different frequencies. Energy resolutions after fitting the $511 \mathrm{keV}$ peak with a Gaussian function are listed in table 4.8.

Table 4.8 Energy resolution, $6 \mathrm{MV}$, different frequencies.

\begin{tabular}{|c|c|c|c|c|}
\hline $6 \mathrm{MV}$ & $50 \mathrm{~Hz}$ & $100 \mathrm{~Hz}$ & $200 \mathrm{~Hz}$ & $400 \mathrm{~Hz}$ \\
\hline FWHM (ch) & 266 & 263 & 274 & 265 \\
\hline XC (ch) & 1681 & 1667 & 1646 & 1596 \\
\hline Energy Res & $16 \%$ & $16 \%$ & $17 \%$ & $17 \%$ \\
\hline
\end{tabular}




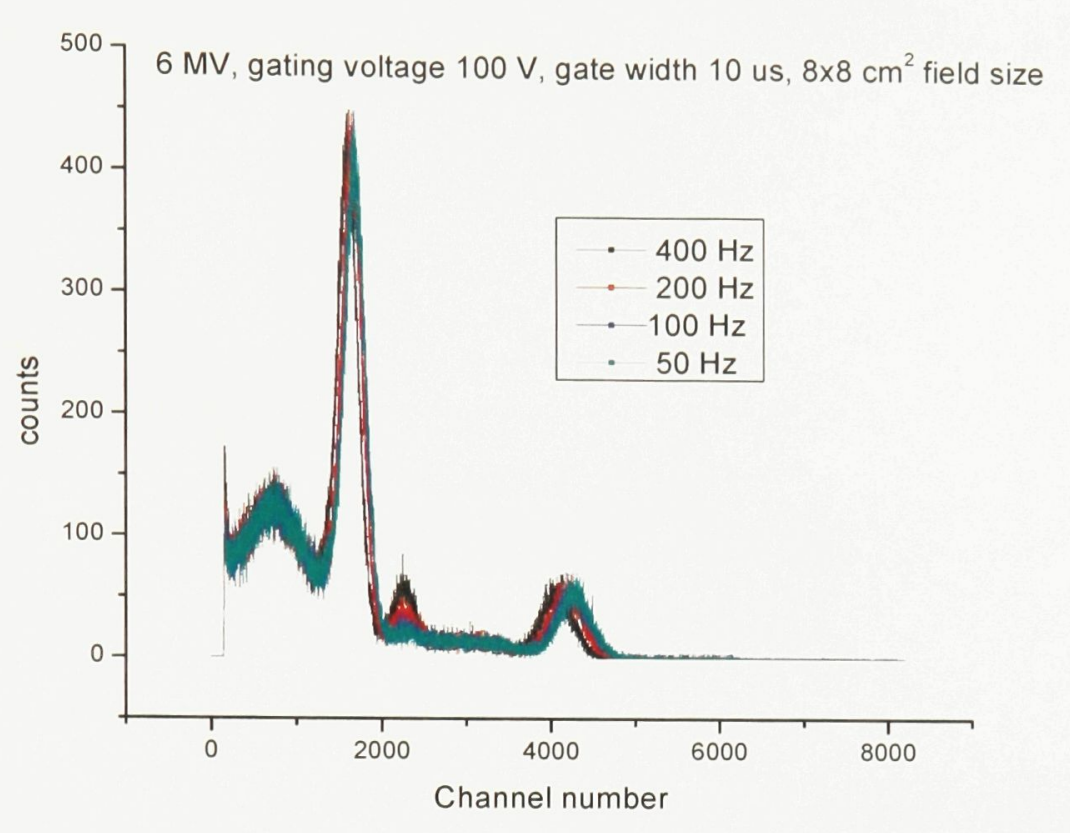

Figure 4.4.2 Energy spectrum of ${ }^{22} \mathrm{Na}$, at $6 \mathrm{MV}$ for different frequencies.

It can be seen that, with gating, energy resolutions of $16 \%$ and $17 \%$ are achieved even for higher frequencies that are comparable to energy resolution of $16 \%$ without gating. The same experiment was repeated for the $10 \mathrm{MV}$ beam and results are shown in figure 4.4.3 and energy resolutions are listed in table 4.9. Energy resolutions of $16 \%$ and $17 \%$ were achieved for the $10 \mathrm{MV}$ beam as well.

Table 4.9 Energy resolution, $10 \mathrm{MV}$, different frequencies.

\begin{tabular}{|c|c|c|c|}
\hline $10 \mathrm{MV}$ & $50 \mathrm{~Hz}$ & $100 \mathrm{~Hz}$ & $200 \mathrm{~Hz}$ \\
\hline FWHM (ch) & 269 & 269 & 269 \\
\hline XC (ch) & 1686 & 1657 & 1623 \\
\hline Eng Res & $16 \%$ & $16 \%$ & $17 \%$ \\
\hline
\end{tabular}




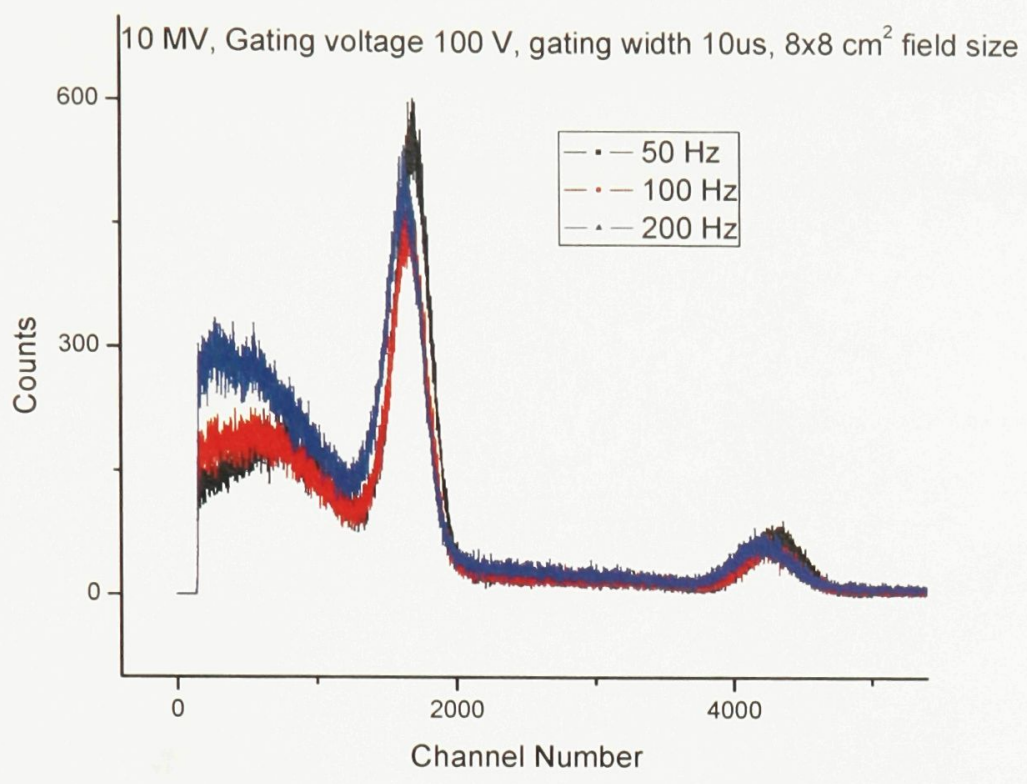

Figure 4.4.3 Energy spectrum of ${ }^{22} \mathrm{Na}$, at $10 \mathrm{MV}$ for different frequencies.

These results indicate that with our optimized gating parameters, the gating circuit is operating in a way that the performance of our detector unit is still as good and system electronics are not as disturbed.

Although it seems that without gating, the scatter radiation from Linac is not disturbing the spectrum, it was observed that intense x-ray scatter saturates the PMT, figure 3.5.3, which will damage the PMT and frontend electronics in the long run [36]. Therefore PMT should be gated off during Linac pulse.

\subsection{Conclusion}

Results of these experiments provided us with strong proof that gating circuit designed was operational with good performance (energy resolution of better than 20\%). The optimal parameters for the gating are found to be, $10 \mu$ s gating width and $100 \mathrm{~V}$ gating voltage. At this point that gating circuit was proved to be working. The very first PeTrack prototype was set up and evaluated. In the following chapter the design and evaluation of this prototype are presented. 


\section{Chapter 5- The first PeTrack Prototype}

After all the primary evaluation of the sample detector unit and the gating circuit it was time to design and build the very first PeTrack prototype.

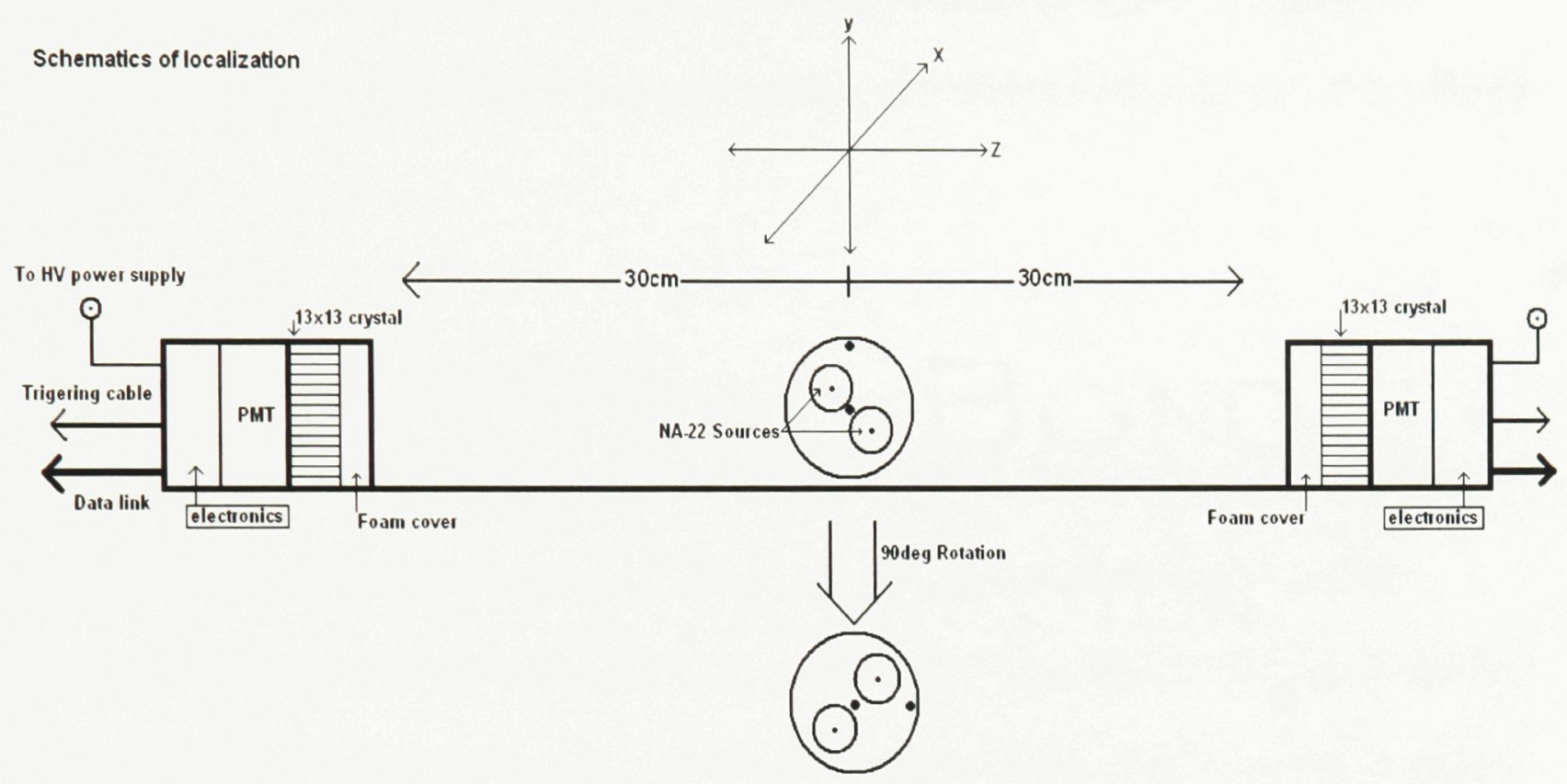

Figure 5.1.1 Schematics of the first PeTrack prototype.

\subsection{Setup of the first PeTrack prototype.}

Two position sensitive PMTs and two crystal blocks were used to set up and calibrate the first PeTrack prototype. Two crystal blocks, $5.2 \times 5.2 \mathrm{~cm}^{2}$, wrapped with Teflon were coupled with two position-sensitive PMTs (Hamamatsu H8500) with silicon grease. The crystal blocks, and PMTs were placed in light tight metal housings. As shown in figure 5.1.1 the two detector units were positioned face to face at a 180 degree angle at a distance of $60 \mathrm{~cm}$.

Mesytec MPET (mesytec GmbH \& Co. KG, Putzbrunn, Germany) is used as the readout system for the PeTrack technique. It includes a fast coincidence and position calculation module (MPET-8) and an intelligence central processing device (MCPD2) which controls 
and buffers the system and transmits the data via Ethernet to a PC. The software used is called Mesydaq and it is the main interface to the PET readout system. Mesydaq controls Mesytec MPET-8 systems and requires a PC with an Ethernet interface. Each Mesytec MPET-8 allows connecting up to 8 position-sensitive photomultipliers, coupled to scintillators (BGO). The PMTs were connected to the MPET-8 through the MPET-H8500 interface.

\section{2- Principle of the readout system}

According to the design of future PeTrack system, there are in total 4 detector module in PeTrack system (Figure 2.1.1: A1, A2, B1, B2). Each detector module includes $2 \times 2$ arrays of BGO crystal blocks $\left(5.2 \times 5.2 \mathrm{~cm}^{2}\right)$ pixelated to $13 \times 13$ arrays of $4 \times 4$ $\mathrm{mm}^{2}$ elements. Each crystal block is coupled with a Hamamatsu H8500 position sensitive photomultiplier. The Mesytec MPET-H8500 interface allows the connection between PMTs and the MPET-8 fast coincidence and position calculation module. As shown in figure 5.2.1 (a), each MPET-8 module allows connecting up to 8 PMTs. The PMTs are grouped in two coincidence groups (A and B) with 4 PMTs each. Only the coincident events between the two groups are accepted. For a full PeTrack system two MPET-8 modules are required. Each pair of opposite detector modules (i.e. A1 and A2 in figure (2.1.1)) is connected to one MPET-8 module. MPET-8 module will convert the four signal outputs from PMT (A, B, C, D) to X and Y location of the energy absorption event Two MPET-8 modules are connected to MCPD2, an intelligence central processing device which controls and buffers the system and transmits the data via Ethernet to a PC (Figure5.2.1 (a), (b)). 

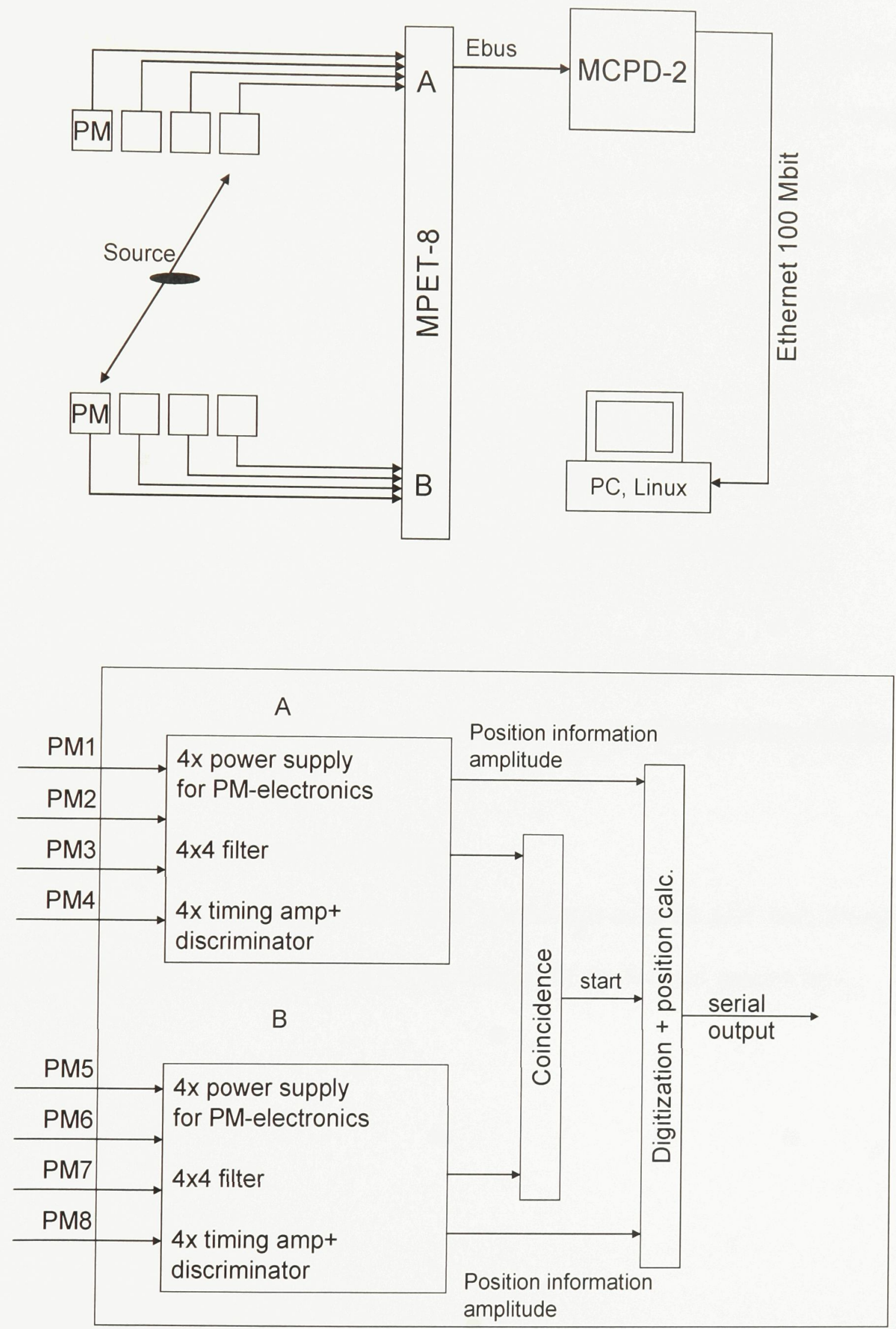

Figure 5.2.1 (a) schematics of the readout system for PeTrack

(b) Schematics of MPET-8 module [31] 
The position of two coincident PMTs as well as their amplitude and also the time shift between the two PMTs are digitized and transmitted via serial bus (coincidence window is $35 \mathrm{~ns}$ ). Conditions on coincidence time and amplitude are adjustable and most of the signal filtering was made via software. The hardware threshold was set to low to allow a software threshold or window setting after software calibration. The following 9 values are transmitted per event:

1 Address of the two responding PMTs (for example 1A, 3B)

2 Amplitude of the two PMT signals (10 bits each)

3 Positions X0, Y0, X1, Y1 of the two responding PMTs (10 bits each)

4 Relative timing of the two PMTs (10 bits)

For the first prototype, there were only two detector units (crystal block + PMT) available. Thus, only one MPET- 8 was used and one detector unit was connected to each coincident group.

\subsection{Calibration of the PeTrack prototype}

As the positron output from the MPET system was in 10 bit ADC unit, the system has to be calibrated to produce the actual hit location of the detected gamma rays. 


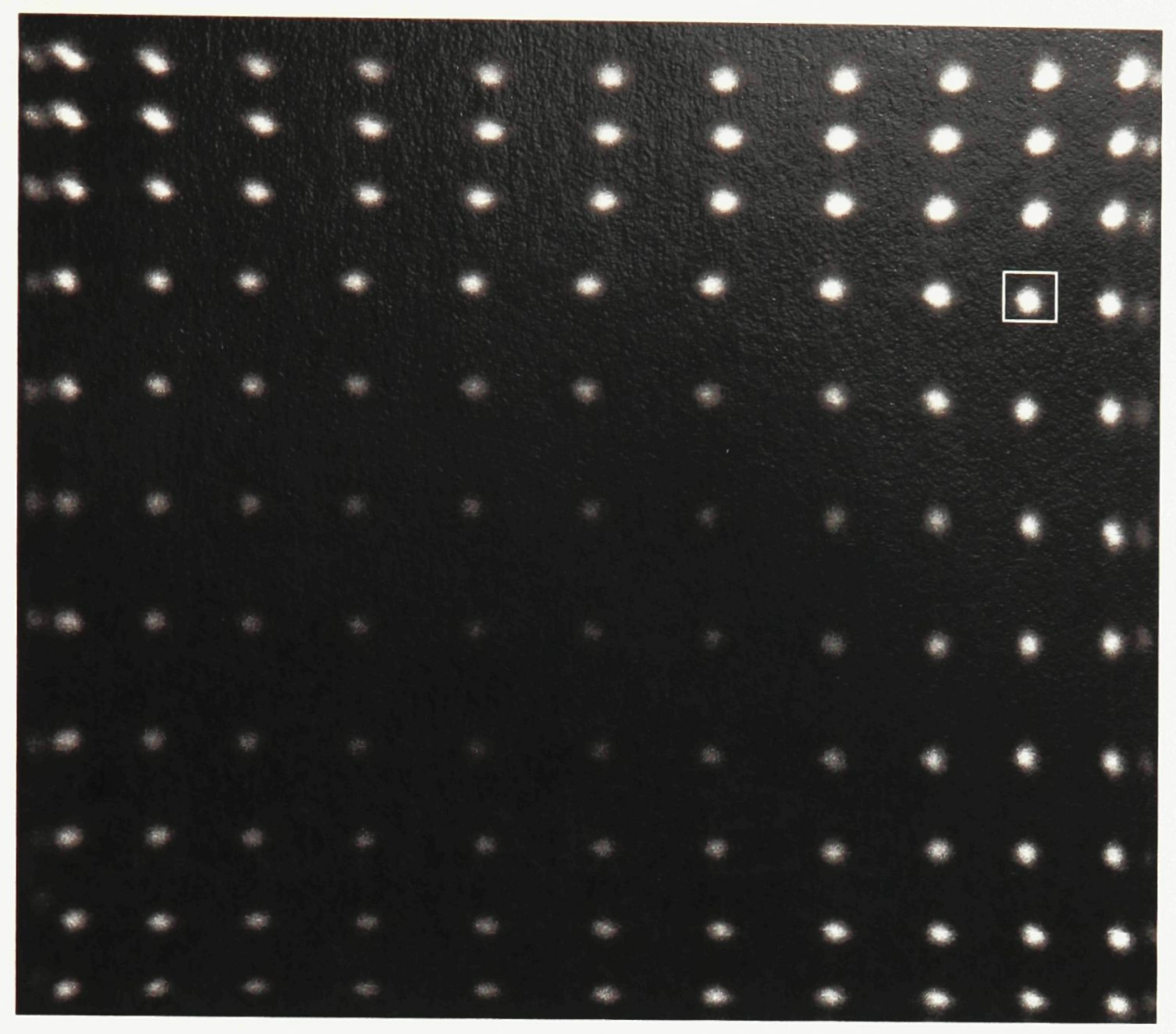

Figure 5.3.1 2D position histogram of PMT \# 0 .

A position emission source, ${ }^{22} \mathrm{Na}$ was placed in the middle of the two detector units in the centre. A $2 \mathrm{D}$ position histogram of detected gamma rays from the ${ }^{22} \mathrm{Na}$ source was generated for each PMT. One of the two histograms is shown in figure 5.3.1. Good crystal pixel identification is clearly visible in these histograms with a good peak to valley ratio. Each bright spot on the $2 \mathrm{D}$ histogram correspond to a crystal element, but it was observed that, due to insensitivity of the PMTs from $3 \mathrm{~mm}$ towards the edges, the last two rows/columns of the crystal elements at the edges of the crystal block are not distinguishable from one another and had to be eliminated in our calibration. Therefore we were left with 9x9 arrays (instead of 13x13) of 4x4 mm² crystal elements (This suggests that in future design of the crystal array, the insensitive areas of the PMT's should be taken into account and a smaller size crystal block should be ordered). 


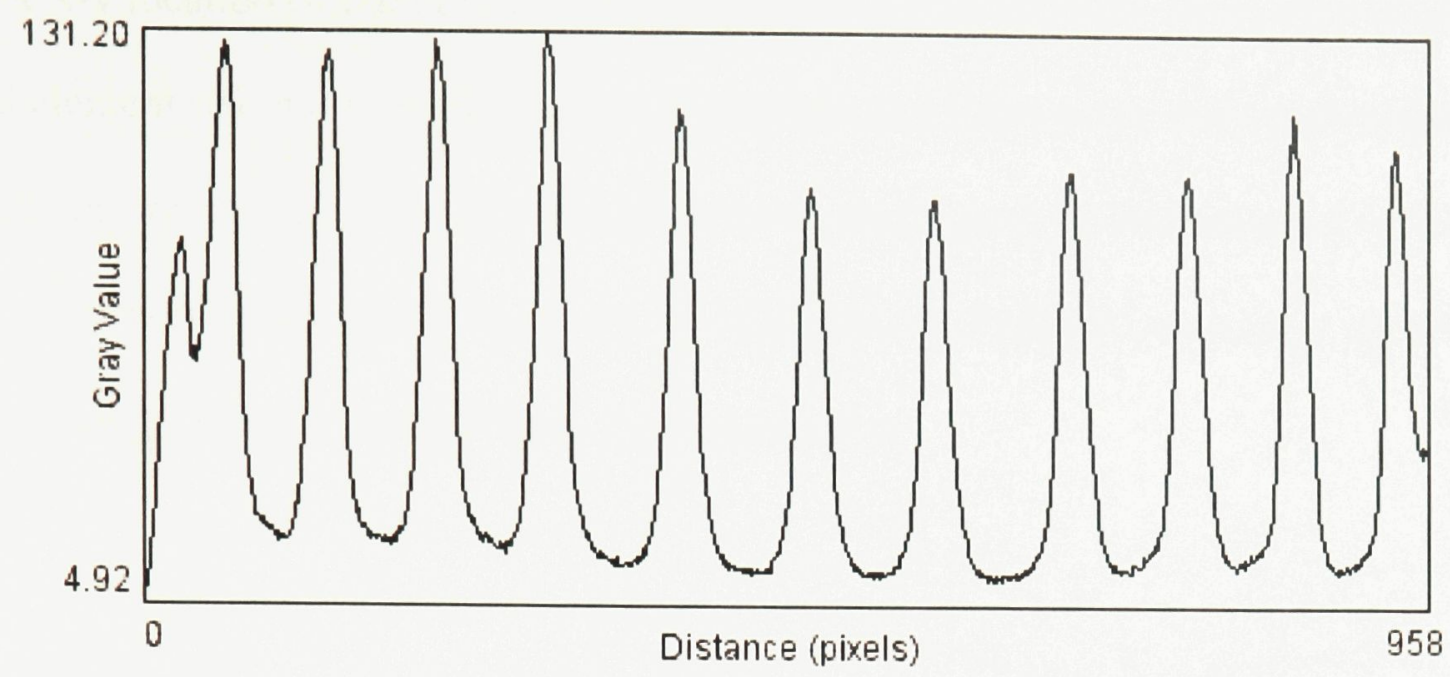

Figure 5.3.2 One dimensional profile of one row of crystal elements

Figure 5.3.2 shows a one dimensional profile of one row of crystal elements. In the profile only 11 crystals out of 13 can be identified. The very last peak on either side of the profile is actually from the last two crystal elements at either end of the row, which were eliminated in the calibration.

Some distortion was observed in the 2D histograms, which is due to the non-uniform gain of the PMT and slight non-linearity introduced by the resistive network readout system [37].

By manually drawing a square region of interest (50x50 pixels) around the peak or each bright spot (ImageJ, Image processing and analysis in Java, National Institute of Health, USA) in the 2D position histogram, figure 5.3.1, the centre mass of the peak was obtained and the coordinates were recorded as the centers of each crystal element. Each crystal element was then given a pair of ID numbers $(i, j)$, i.e. i-th row and $j$-th column. The data file obtained from the Mesydaq software consists of the $x-y$ location of each event. These $\mathrm{x}-\mathrm{y}$ locations are in ADC units. To convert these $\mathrm{x}-\mathrm{y}$ locations to crystal 
IDs, the $x-y$ location of each event was compared to $x-y$ locations of the centers of all crystal elements (found from $2 \mathrm{~d}$ position histogram) and if the event was inside a square of 50 pixels around the centre of a crystal element, that crystal ID was assigned to the event. After converting all x-y locations, in ADC unit, to crystal ID, an energy spectrum for each crystal was generated, figure 5.3.3, a, b and c.
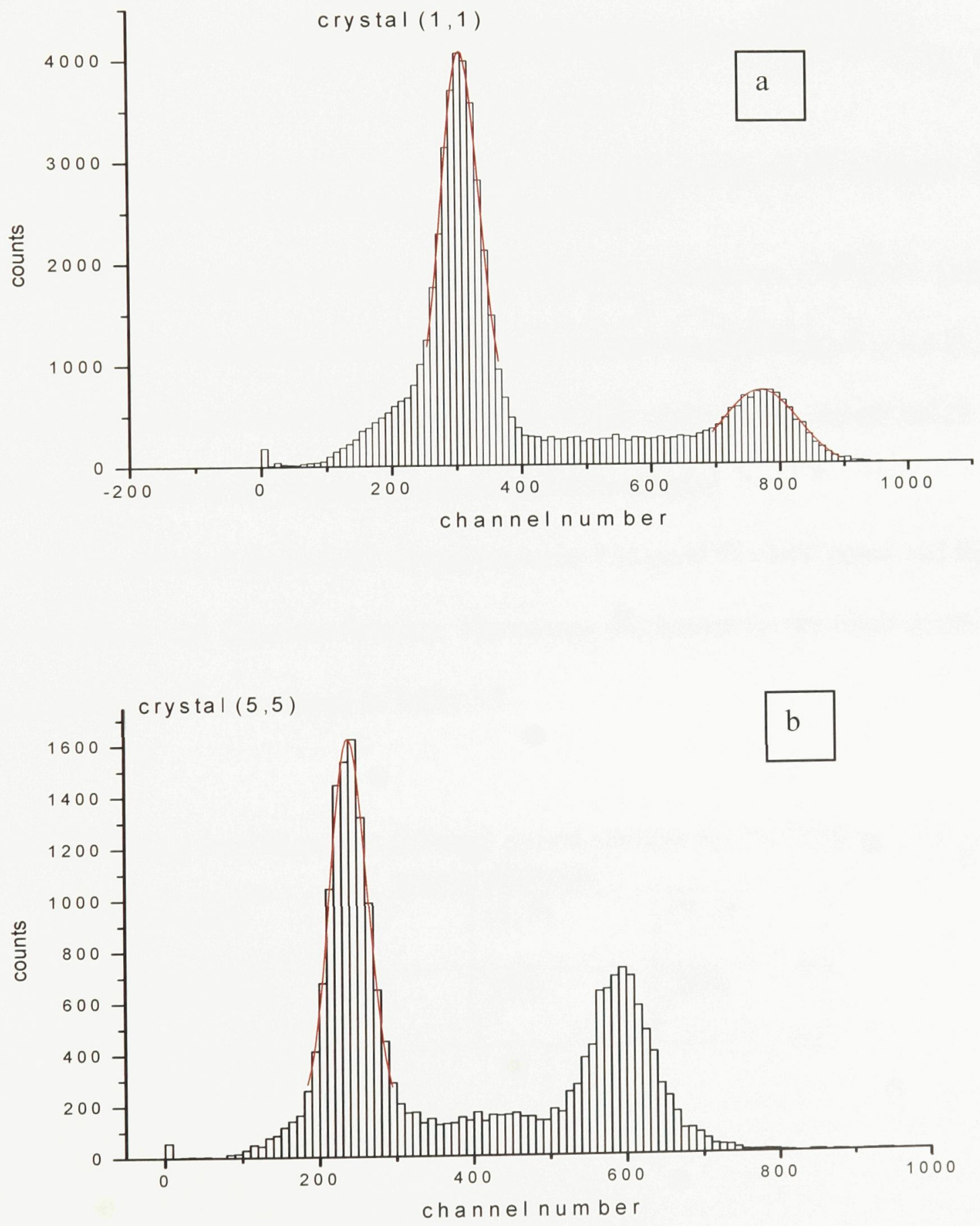


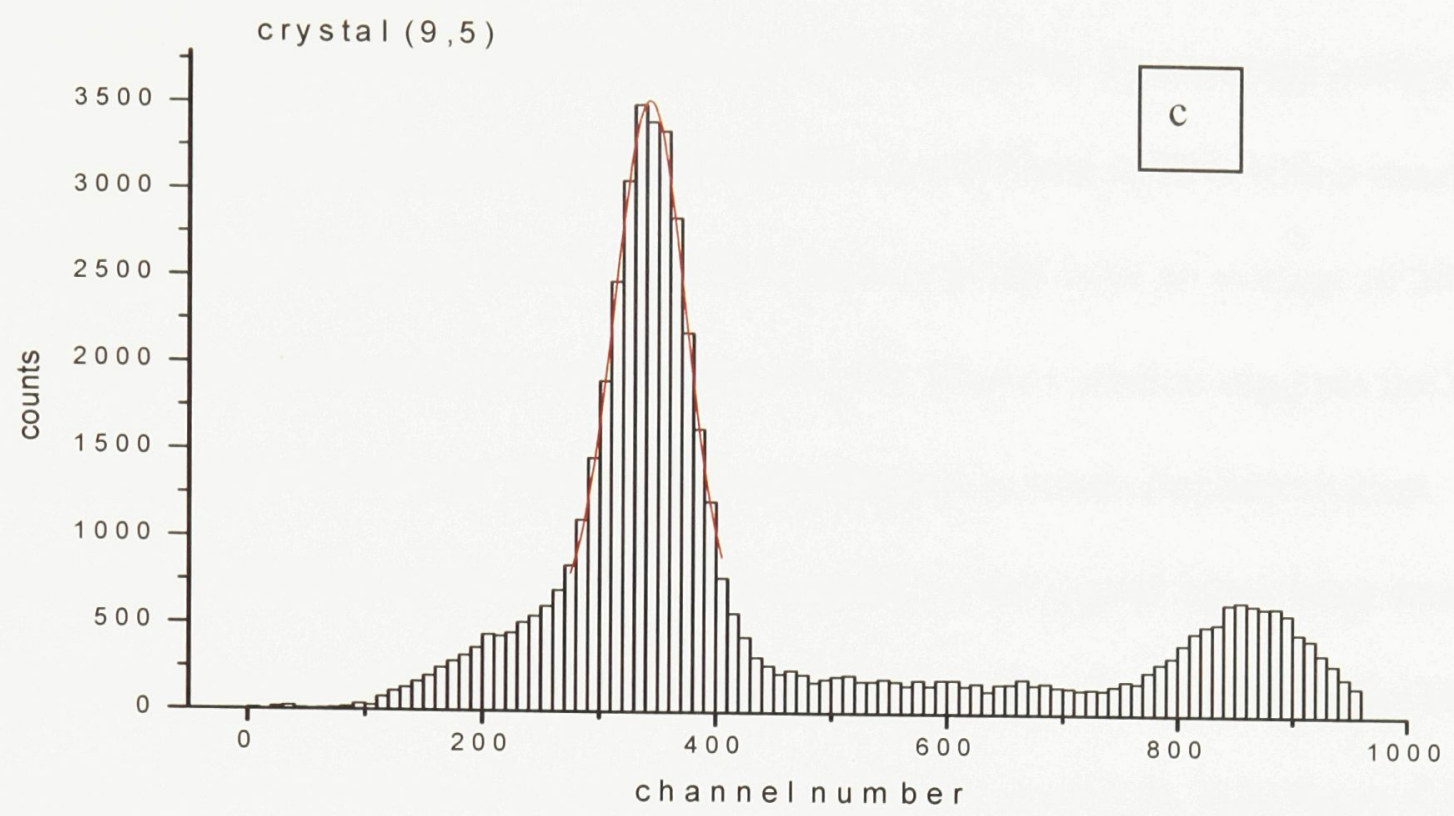

Figure 5.3.3 Energy spectrum of $4 \times 4 \mathrm{~mm}^{2}$ crystal elements. (a) crystal ID number $(1,1)$, (b) crystal ID number $(5,5)$, and (c) crystal ID number $(9,5)$

In figure 5.3.3 energy spectra of different crystal elements are shown. Crystal $(1,1)$ is located in the first row, and the first column (top left of the crystal block). Crystal $(5,5)$ is located in the fifth row and fifth column, in the middle of the block, and crystal $(9,5)$ is located in the middle at the bottom, ninth row and fifth column.

Energy spectra obtained from all 81 crystal elements had good $511 \mathrm{keV}$ peaks and these peaks were fitted with Gaussian function. The energy resolutions for the three crystal elements in figure 5.3.3 are listed in Table 5.1.

Table 5.1 Energy resolutions for different crystal element in a $9 \times 9$ array of $4 \times 4 \mathrm{~mm}^{2}$ crystal elements

\begin{tabular}{|l|l|l|l|}
\hline Crystal element & $(1,1)$ & $(5,5)$ & $(9,5)$ \\
\hline Energy resolution & $22 \%$ & $23 \%$ & $20 \%$ \\
\hline
\end{tabular}


It can be seen from the table that crystal elements at different locations of the crystal block have almost the same energy resolution of about 20-23\%. The average energy resolution for 10 crystal elements sampled from the crystal block is $22 \%$ with a standard deviation of $1 \%$ and the location of the $511 \mathrm{keV}$ gamma peaks have an average of 284 channels with a standard deviation of 32 channels. The above variation suggests the use of different energy windows for different crystal elements in future implementation. It was also observed that crystal elements in the middle of the crystal block have smaller energy peaks compared with the elements located closer to the edges of the block (1600 for crystal $(5,5)$ versus 4000 for crystal $(1,1)$ and 3500 for crystal $(9,5)$ in figure 5.3.3). This variation is due to the gain non-uniformity across the position-sensitive PMT. The gain at the center of the PMT is lower (lower peak locations in the spectrum) than the gain at the edges. As a result there are fewer events passing the triggering threshold at the center (triggering is based on the dynode signal instead of the sum of the anode signals).

The next step in our calibration was to find the true location of each event in Cartesian coordinates. To do this, first the true locations of the centers of all crystal elements, based on the geometry of our system, were measured and recorded. Later, the crystal ID that was attached to each event was converted to the $x-y-z$ Cartesian coordinates of the true location of the centre of the crystal element. After doing all these conversions, data are in a suitable format for PeTrack algorithm. The PeTrack algorithm uses the endpoint coordinates of the recorded coincidence lines to localize the markers. 


\subsection{Evaluation of the PeTrack prototype}

After the calibration, the following setup was arranged to evaluate the localization ability of PeTrack prototype and algorithm. As shown in figure 5.1.1, two ${ }^{22} \mathrm{Na}$ sources were placed $2.54 \mathrm{~cm}$ away from each other on a rotating disk. Both data acquisition systems were set to only record the coincidence events (coincidence window was open for $35 \mathrm{~ns}$ ). As described in Chapter 2, to do 3D localization we need to have two pairs of orthogonal detector modules. However, we had only one pair available for the first prototype. To overcome this problem, we had to take the first set of data, then either rotate the detectors around the sources by 90 degrees (clockwise), or rotate the sources by 90 degrees (anti clockwise), and acquire the second set of data.

After the acquisition of first set of data, the disk, i.e. sources, were rotated 90 degree and the second set of data was acquired. The two data sets were combined and randomly mixed to generate a single data set as if the events were acquired from two pair of detectors simultaneously.

The data were first converted to crystal ID (as explained in section 5.3, calibration process) and then to true location of the events in Cartesian coordinates. Later these Cartesian coordinates of the events were fed into the PeTrack algorithm (section 2.5).

\subsection{Result}

A total of 5528 events were captured and 200 lines were used for each run of localization. After rejecting the scatter and random events, on average 88 lines were used to localize each source for each run. The 5528 events allow us to do $27(5528 / 200)$ runs of localization. The precision was assessed by the standard deviation among the 27 
localization runs. The average precision of both sources were $0.16 \mathrm{~mm}$ on $\mathrm{x}, 0.2 \mathrm{~mm}$ on $\mathrm{y}$, and $0.2 \mathrm{~mm}$ on $\mathrm{z}$. The average distance between the two sources calculated by the algorithm was $24.78 \mathrm{~cm} \pm 0.25 \mathrm{~mm}$, compared with the true distance of $25.4 \mathrm{~mm}$, gives us a localization accuracy of $0.6 \mathrm{~mm}$ (this localization accuracy will be better once we reduce the systematic errors, i.e. misalignment of the detector units and uncertainty of 0.5 $\mathrm{mm}$ on the location of the crystal block inside the detector module due to wrapping and the cushions around the crystal blocks).

\subsection{Conclusions}

The First PeTrack prototype was successfully set up with only two pairs of detector modules. The data acquisition system was working properly and the system was calibrated. The prototype was able to localize two positron emitting sources with submillimeter precision and accuracy. 


\section{Chapter 6- Conclusion and future work}

Experiments and results presented in this work introduce and support the PeTrack system as an innovative tumor tracking technique. The PeTrack system has the ability to track tumor's motion in real-time using positron emission markers with sub-millimeter accuracy.

The objective of this work was to develop and evaluate the detectors for PeTrack. To accomplish this, many experiments were performed and a summary of them is provided here.

- BGO crystal was evaluated and the results obtained confirmed that BGO is a suitable scintillating crystal for PeTrack position sensitive gamma ray detectors.

- Detector units were built and evaluated under the radiation environment of a clinical linear accelerator and results for energy resolution were within the acceptable energy resolution range (better than $25 \%$ ).

- A gating circuit was designed, built and evaluated. The results obtained confirmed the benefit of using a gating circuit. The optimal gating parameters were determined.

- The readout system of the PeTrack was set up and calibrated successfully.

- Finally, the very first PeTrack prototype was designed, built and evaluated. Data produced from readout system after calibration were fed into the PeTrack algorithm for localization. Sub-millimeter precession and accuracy was achieved. The encouraging results conducted from the very first PeTrack Prototype have motivated the commercial production of this technique. Future work is going to be focused on building the first engineering prototype. 


\section{References}

1) Tong Xu, Jerry T. Wong, Polad M Shikhaliev, and Justin L. Ducote, Real-time tumor tracking using implanted positron emission markers: concept and stimulation. Med. Phys. 2006. 33(7): p.2598-2609.

2) S.C. Davies, A.L. Hill, R.B. Holmes, M. Halliwell, and P.C. Jackson, Ultrasound quantification of respiratory organ motion in the upper abdomen. Br J Radiol, 1994. 67(803): p. 1096-102.

3) C.S. Ross, D.H. Hussey, E.C. Pennington, W. Stanford, and J.F. Doornbos, Analysis of movement of intrathoracic neoplasms using ultrafast computerized tomography. Int. J. Radiat Oncol Biol Phys, 1990. 18(3): p. 671-7.

4) IMRT-CWG, Intensity-Modulated Radiotherapy: Current Status and Issues of Interest. Int. J. Radiation Oncology Biol. Phys., 2001. 51(4): p. 880-914.

5) T.R. Mackie, T. Holmes, S. Swerdloff, P. Reckwerdt, JO. Deasy, J. Yang, B. Paliwal, T. Kinsella, Tomotherapy: a new concept for the delivery of dynamic conformal radiotherapy. Medical Physics, 1993. 20(6): p. 1709-19.

6) C.X. Yu, Intensity-modulated arc therapy with dynamic multileaf collimation: an alternative to tomotherapy. Physics in Medicine and Biology, 1995. 40(9): $\mathrm{p}$. 1435-49.

7) J.S. Kuo, C. Yu, Z. Petrovich, and M. I. Apuzzo, The Cyberknife stereotactic radiosurgery system: description, installation, and an initial evaluation of use and functionality. Neurosurgery, 2003. 53(5): p. 1235-9; discussion 1239.

8) C.C. Ling, J. Humm, S. Larson, H. Amols, Z. Fuks, S. Leibel, JA Koutcher, Towards multidimensional radiotherapy (MD-CRT): biological imaging and 
biological conformality. Int. J. Radiat. Oncol. Biol. Phys, 2000. 47(3): p. 551-560.

9) G.Starkschall, KM. Forester, K. Kitamura, A. Cardenas, SL. Tucker, CW.

Stevens, Correlation of gross tumor volume excursion with potential benefits of respiratory gating. Int. J. Radiat Oncol Biol Phys, 2004.60(4): p. 1291-7.

10) N. Koch, H.H. Liu and G. Starkschall et al., Evaluation of internal lung motion for respiratory-gated radiotherapy using MRI: Part I-correlating internal lung motion with skin fiducial motion. Int. J. Radiat Oncol Biol Phys, 2004. 60(5): p. $1459-72$.

11) J.D. Hoisak, K.E. Sixel, R. Tirona, P.C. Cheung, and J.P. Pignol, Correlation of lung tumor motion with external surrogate indicators of respiration. Int. J. Radiat Oncol Biol Phys, 2004. 60(4): p. 1298-306.

12) C. Ozhasoglu and M.J. Murphy, Issues in respiratory motion compensation during external-beam radiotherapy. Int. J. Radiat. Oncol. Biol. Phys, 2002. 52(5): p. 1389-99.

13) R.I. Berbeco, S. Nishioka, H. Shirato, G.T. Chen, and S.B. Jiang, Residual motion of lung tumors in gated radiotherapy with external respiratory surrogates. Phys Med Biol, 2005. 50(16): p. 3655-67.

14) M. J. Murphy, Image-guided motion adaption in radiotherapy, Department of Radiation Oncology, Virginia Commonwealth University, Richmond, VA, 2007.

15) H. Shirato, T. Kunieda, K. Kitamura, M. Herk, K. Kagei, T. Nishioka, et al., Physical aspects of a real-time tumor tracking system for gated radiotherapy. Int. J. Radiat Oncol Biol Phys, 2000. 48(4): p. 1187-95.

16) J.M. Balter, J. N. Wright, L. J. Newell, B. Friemel, S. Dimmer, Y. Cheng, J. 
Wong, E. Vertatschitsch, and T. P. Mate, Accuracy of a wireless localization system for radiotherapy. International Journal of Radiation Oncology Biology Physics, 2005. 61(3): p. 933-937.

17) H. Shirato, M. Oita, K. Fujita, Y. Watanabe, and K. Miyasaka, Feasibility of synchronization of real-time tumor-tracking radiotherapy and intensitymodulated radiotherapy from viewpoint of excessive dose from fluoroscopy. Int. J. Radiat Oncol Biol Phys, 2004. 60(1): p. 335-41.

18) T. Neicu, H. Shirato, Y. Seppenwoolde, and S. B. Jiang, Synchronized moving aperture radiation therapy (SMART): average tumor trajectory for lung patients. Phys Med Biol, 2003. 48(5): p. 587-98.

19) L. Papiez, D. Rangaraj, and P. J. Keall, Real-time DMLC IMRT delivery for mobile and deforming targets. Med. Phys., 2005. 32(9): p. 3037-3048.

20) S.B. Koebel and P.L. McHenry, Myocardial blood flow. Measurement by a coincidence counting system and single bolus of ${ }^{84} R b$. Arch Intern. Med, 1971. 127(4): p. 767-72.

21) D. Boettcher, G. Corsini, C. G. Daniels, C. Cowan, and R.J. Bing, Determination of myocardial blood flow in the anesthetized dog after a bolus of ${ }^{84} R b$. J. Nucl. Med, 1969. 10(2): p. 83-92.

22) J.D. Kimpe, R. Cornelis, L. Mees, and R. Vanholder, Basal metabolism of intraperitoneally injected carrier-free ${ }^{74}$ As-labeled arsenate in rabbits. Fundam Appl Toxicol, 1996. 34(2): p. 240-8.

23) S. Robinson, P.J. Julyan, D.L. Hastings, and J. Zweit, Performance of a block detector PET scanner in imaging non-pure positron emitters--modeling and 
experimental validation with ${ }^{124}$ I. Phys Med Biol, 2004. 49(24): p. 5505-28.

24) R. Y. Zhu, H. Stone, H. Newman, T. Q. Zhou, H. R. Tan, and C. F. He, A study on Radiation-Damage in Doped BGO crystal. Nuclear Instruments \& Methods in Physics Research Section A-Accelerators Spectrometers Detectors and Associated Equipment, 1991. 302(1): p. 69-75.

25) S. K. Sahu, K. C. Peng, H. C. Huang, C. H. Wang, Y. H. Cheng, W. S. Hou, K. Ueno, F. I. Chou, and Y. Y. Wei, Radiation hardness of undoped BGO crystals. Nuclear Instruments \& Methods in Physics Research Section A-Accelerators Spectrometers Detectors and Associated Equipment, 1997. 388(1-2): p. 144-148.

26) K. C. Peng, Z. M. Wang, C. H. Wang, K. Ueno, H. C. Huang, Y. H. Chang, F. I. Chouf, W. S. Hou, and Y. Y. Wei, Performance of undoped BGO crystals under extremely high dose conditions. Nuclear Instruments \& Methods in Physics Research Section A-Accelerators Spectrometers Detectors and Associated Equipment, 1999. 427(3): p. 524-527.

27) Z. Y. Wei, R. Y. Zhu, H. Newman, and Z.W. Yin, Radiation-Resistance and Fluorescence of Europium Doped BGO crystals. Nuclear Instruments \& Methods in Physics Research Section A-Accelerators Spectrometers Detectors and Associated Equipment, 1990. 297(1-2): p. 163-168.

28) R. Engels, U. Clemens, G Kemmerling, and J. Schelten, High Spatial Resolution Scintillation Detector based on the H8500 Photomultiplier. Nuclear Science Symposium Conference Record, 2003. 1: p. 692-695.

29) D. J. Parker, C. J. Broadbent, P. Fowles, M. R. Hawkesworth, and P. Mcneil, Positron Emission Particle Tracking-A technique for studying flow within 
engineering equipment. Nuclear Instruments \& Methods in Physics Research Section A-Accelerators Spectrometers Detectors and Associated Equipment, 1993.326(3): p. 592-607.

30) D. J. Parker, R. N. Forster, P. Fowles, and P. S. Takhar, Positron Emission Particle Tracking using the new Birmingham positron camera. Nuclear Instruments \& Methods in Physics Research Section A-Accelerators Spectrometers Detectors and Associated Equipment, 2002. 477(1-3): p. 540-545.

31) O. Gundogdu, Positron emission tomography particle tracking using cluster analysis. Nuclear Instruments \& Methods in Physics Research Section AAccelerators Spectrometers Detectors and Associated Equipment, 2004. 534(3): p. $562-576$.

32) MPET-8 main module manual, V1.3, Mesytec GmbH \& co KG, Putzbrunn, Germany, 2008.

33) J. Gironnet, V.B. Mikhailik, H. Kraus, P.de Marcillac, N.Coron, Scintillation studies of $B G O$ down to a temperature of $6 \mathrm{~K}$. Nuclear Instruments \& Methods in Physics Research A 2008. 594: p. 358-361.

34) T. Nakamoto, Y. Fukazawa, T. Ohsugi, T. Kamae, and J. Kataoka, BGO readout with photodiodes as a soft gamma-ray detector at $-30^{\circ} \mathrm{C}$. Nuclear Instruments \& Methods in Physics Research Section A-Accelerators Spectrometers Detectors and Associated Equipment, 2005. 536(1-2): p. 136-145.

35) G. C, Santana, A. C. S. de Mello, M. E. G. Valerio, Z. S. Macedo, Scintillating properties of pure and doped BGO ceramics. J Mater Sci, 2007. 42: p. 2231-2235.

36) R. Wardle, Electron Tubes Ltd., technical reprint R/P061 gating of 
photomultipliers, Ruislip, UK. 1973.

37) Pedro Guerra, Modeling the Acquisition front-end in high resolution gamma-ray imaging. IEEE Transactions on Nuclear Science, 2006. 53: No. 3, p. 1150-56.

38) The management of respiratory motion in radiation oncology Report of AAPM Task Group 76. Med. Phys. 33, Issue 10, p. 3874-3900 (October 2006).

39) Photomultipliers handbook, basics and application, third edition, p. 167-171, Hamamatsu Co., Shizuoka, Japan, 2006. 